TRANSACTIONS OF THE

AMERICAN MATHEMATICAL SOCIETY

Volume 356, Number 5, Pages 1833-1874

S 0002-9947(03)03346-

Article electronically published on August 26, 2003

\title{
POINCARÉ SERIES OF RESOLUTIONS OF SURFACE SINGULARITIES
}

\author{
STEVEN DALE CUTKOSKY, JÜRGEN HERZOG, AND ANA REGUERA
}

ABstRACT. Let $X \rightarrow \operatorname{spec}(R)$ be a resolution of singularities of a normal
surface singularity $\operatorname{spec}(R)$, with integral exceptional divisors $E_{1}, \ldots, E_{r}$. We
consider the Poincaré series
\[ g=\sum_{\underline{n} \in \mathbf{N}^{r}} h(\underline{n}) t \underline{n} \]

where

$$
h(\underline{n})=\ell\left(R / \Gamma\left(X, \mathcal{O}_{X}\left(-n_{1} E-1-\cdots-n_{r} E_{r}\right)\right) .\right.
$$

We show that if $R / m$ has characteristic zero and $\operatorname{Pic}^{0}(X)$ is a semi-abelian variety, then the Poincaré series $g$ is rational. However, we give examples to show that this series can be irrational if either of these conditions fails.

\section{INTRODUCTION}

Suppose that $R$ is an excellent, normal local ring of dimension 2, with maximal ideal $m$. Let

$$
f: X \rightarrow \operatorname{spec}(R)
$$

be a resolution of singularities, with integral exceptional divisors $E_{1}, \ldots, E_{r}$.

If $\underline{n}=\left(n_{1}, \ldots, n_{r}\right) \in \mathbf{N}^{r}$, let

$$
D_{\underline{n}}=\sum_{i=1}^{r} n_{i} E_{i} .
$$

$\Gamma\left(X, \mathcal{O}_{X}\left(-D_{\underline{n}}\right)\right) \subset R$ is an ideal, and $R / \Gamma\left(\left(X, \mathcal{O}_{X}\left(-D_{\underline{n}}\right)\right)\right.$ has finite length as an $R$-module. Consider the function

$$
h(\underline{n})=\ell\left(R / \Gamma\left(\left(X, \mathcal{O}_{X}\left(-D_{\underline{n}}\right)\right)\right)\right.
$$

and the Poincaré series

$$
g=\sum_{\underline{n} \in \mathbf{N}^{r}} h(\underline{n}) t^{\underline{n}},
$$

where $t^{\underline{n}}=t_{1}^{n_{1}} \ldots t_{r}^{n_{r}}$ is a monomial in the variables $t_{1}, \ldots, t_{r}$.

By the local Riemann Roch theorem (cf. (6))

$$
h(\underline{n})=(\text { quadratic polynomial in } \underline{n})-h^{1}\left(X, \mathcal{O}_{X}\left(-D_{\underline{n}}\right)\right) .
$$

Received by the editors August 1, 2002.

2000 Mathematics Subject Classification. Primary 14B05, 14F05, 13A30.

The first author's research was partially supported by NSF.

(C)2003 American Mathematical Society 
Thus the Poincaré series

$$
g=(\text { rational series in } \underline{n})-\sum_{\underline{n} \in \mathbf{N}^{r}} h^{1}\left(X, \mathcal{O}_{X}\left(-D_{\underline{n}}\right)\right) t \underline{n} .
$$

In this paper we consider the form of the function $h(\underline{n})$, and the question of the rationality of $g$.

If $r=1$, the situation is very simple, as $-D_{1}$ is ample, so that

$$
h\left(n_{1}\right)=\text { quadratic polynomial in } n_{1} \text { for } n_{1}>>0
$$

and $g$ is thus rational. In this case $(r=1) \bigoplus_{n \geq 0} \Gamma\left(X, \mathcal{O}_{X}\left(-n_{1} D_{1}\right)\right)$ is a finitely generated $R$-algebra, so $h\left(n_{1}\right)$ is a Hilbert polynomial (for $n_{1}>>0$ ).

If $r>1$, the behavior of $h(\underline{n})$ is much more subtle. We first observe that if $(\mathbf{k}=R / m$ is algebraically closed of characteristic zero and) $R$ is not a rational singularity, then there exists a resolution $f: X \rightarrow \operatorname{spec}(R)$ such that $\bigoplus_{\underline{n} \in \mathbf{N}^{r}} \Gamma\left(X, \mathcal{O}_{X}\left(-D_{\underline{n}}\right)\right)$ is not a finitely generated $R$-algebra, so we might not expect polynomial-like behavior of $h(\underline{n})$, or rationality of the Poincaré series $g$. To see this, we first observe that with our assumptions, $R$ has a rational singularity if and only if the divisor class group $\mathrm{Cl}(R)$ of $R$ is a torsion group (as follows from [2] and [19]). By Theorem 4 of [8], if $\mathrm{Cl}(R)$ is not torsion, then there exist a resolution $f: X \rightarrow \operatorname{spec}(R)$ and an exceptional divisor $F$ on $X$ such that $\bigoplus_{n>0} \Gamma\left(X, \mathcal{O}_{X}(-n F)\right)$ is not a finitely generated $R$-algebra. Thus the ring $\bigoplus_{\underline{n} \in \mathbf{N}^{r}} \Gamma\left(X, \mathcal{O}_{X}\left(-D_{\underline{n}}\right)\right)$ is not a finitely generated $R$-algebra.

If $r>1$, then even in the best cases $h^{1}\left(X, \mathcal{O}_{X}\left(-D_{\underline{n}}\right)\right)$ is a complicated function. If $-D_{\underline{\underline{n}}}$ is sufficiently ample, then $h^{1}\left(X, \mathcal{O}_{X}\left(-D_{\underline{n}}\right)\right)$ will vanish. More generally, $h^{1}\left(X, \overline{\mathcal{O}}_{X}\left(-D_{\underline{n}}\right)\right)$ will tend to be small if $D_{\underline{n}}$ is in the cone $\mathbf{E}^{+}$of exceptional curves $D$ such that $-D$ is nef $\left(\left(D \cdot E_{i}\right) \leq 0\right.$ for all $\left.i\right)$. To be precise, $h^{1}\left(X, \mathcal{O}_{X}\left(-D_{\underline{n}}\right)\right)$ is bounded for $D_{\underline{n}} \in \mathbf{E}^{+}$(as follows from Lemma 2.4). On the other hand, $h^{1}\left(X, \mathcal{O}_{X}\left(-D_{\underline{n}}\right)\right)$ will tend to be large if $D_{\underline{n}}$ is far outside of $\mathbf{E}^{+}$.

We prove (proposition 6.3) that there exists an "abstract complex of polyhedral sets" $\mathcal{P}$, whose union is $\mathbf{Q}_{\geq 0}^{r}$, such that, for $P \in \mathcal{P}$ and $\underline{n} \in P \cap \mathbf{N}^{r}$,

$$
h(\underline{n})=Q(\underline{n})+L(\underline{n})+\varphi(\underline{n}),
$$

where $Q(\underline{n})$ is a quadratic polynomial, $L(\underline{n})$ is a linear function with periodic coefficients (that is, $L(\underline{n})=\sum_{i=1}^{r} \varphi_{i}(\underline{n}) n_{i}$ where the $\varphi_{i}(\underline{n})$ are periodic functions), and $\varphi(\underline{n})$ is a bounded function.

To construct $\mathcal{P}$ we first construct a fan $\Sigma$ subdividing $\mathbf{Q}_{\geq 0}^{r}$ on which the Zariski decomposition (see Proposition 2.1) is a linear function on each cone in $\Sigma$. The fan $\Sigma$ is determined by the "shadow" of the cone $\mathbf{E}^{+}$from $E_{1}, \ldots, E_{r}$. The abstract complex of polyhedral sets $\mathcal{P}$ is defined by refining $\Sigma$ in such a way that we have good properties for the divisor $\bar{D}_{\underline{n}}$ (called the Laufer divisor in [7]) associated to $D_{\underline{n}}$, which is determined by the properties that $D_{\underline{n}} \leq \bar{D}_{\underline{n}}, \bar{D}_{\underline{n}} \in \mathbf{E}^{+}$and $H^{0}\left(\bar{X}, \mathcal{O}_{X}\left(-\bar{D}_{\underline{n}}\right)\right)=H^{0}\left(X, \mathcal{O}_{X}\left(-D_{\underline{n}}\right)\right)$. Then, the essential contribution to $\varphi(\underline{n})$ is $h^{1}\left(X, \mathcal{O}_{X}\left(-\bar{D}_{\underline{n}}\right)\right)$.

In the case where $\operatorname{spec}(R)$ is a rational singularity, we have that $H^{1}\left(X, \mathcal{O}_{X}\left(-\bar{D}_{\underline{n}}\right)\right)$ $=0$ for all $\underline{n}[19]$, so that $\varphi(\underline{n})=0$ is a periodic function (on the abstract complex $\mathcal{P})$, and we conclude that the series $g$ is rational.

Therefore, we are reduced to understanding the function $h^{1}\left(X, \mathcal{O}_{X}\left(-\bar{D}_{\underline{n}}\right)\right.$. 
When $r=2$ and $k=R / m$ has characteristic zero, this function has a very nice form. $h^{1}\left(X, \mathcal{O}_{X}\left(-\bar{D}_{\underline{n}}\right)\right)$ is in fact semi-periodic (Definition 6.5) on an abstract complex of polyhedral sets, and the series $g$ is thus rational. This is the case $r=2$ of Theorem 6.6 and Theorem 7.7. The proof is a generalization of Theorem 9 in [9].

An example showing a nontrivial (but semi-periodic) function $h^{1}\left(X, \mathcal{O}_{X}\left(-\bar{D}_{\underline{n}}\right)\right)$ is given in section 10 .

When $r=2$ and $R$ contains a field $k$ of characteristic $p>0$, we give an example where $h^{1}\left(X, \mathcal{O}_{X}\left(-\bar{D}_{\underline{n}}\right)\right.$ is not semi-periodic and $g$ is not rational in section 8 . This is an extension of example 5 in $[9$.

In section 9 we give an example of $X \rightarrow \operatorname{spec}(R)$ such that $r=3$ and $\mathbf{k}=R / m$ has characteristic $0, h^{1}\left(X, \mathcal{O}_{X}\left(-\bar{D}_{n}\right)\right)$ is not semi-periodic and $g$ is not rational. This example is obtained by first constructing a resolution $X_{1} \rightarrow \operatorname{spec}(R)$ where $X_{1}$ has only a single irreducible exceptional divisor $(r=1)$, and then blowing up 2 points on $X_{1}$ to construct $X \rightarrow \operatorname{spec}(R)$. Thus the function $h$ is semi-periodic on $X_{1}$, and the resulting Poincaré series $g$ on $X_{1}$ is rational. In particular, we see that the rationality of $g$ depends on the resolution of $\operatorname{spec}(R)$.

In Theorem[6.6] and Theorem[7.7 we prove that if $\mathbf{k}=R / m$ is algebraically closed of characteristic zero, and the group of numerically trivial line bundles $\operatorname{Pic}^{0}(X)$ on $X$ is a semi-abelian variety, then $h^{1}\left(X, \mathcal{O}_{X}\left(-\bar{D}_{\underline{n}}\right)\right)$ is semi-periodic (in an abstract complex of polyhedral sets) and $g$ is rational. A semi-abelian variety is an extension of an abelian variety by a product of multiplicitive groups $\mathbf{G}_{m}$. $\operatorname{Pic}(X)$ is semiabelian if and only if the divisor class group of $\mathrm{Cl}(R)$ is an extension of a finite group by a semi-abelian variety, since $\mathrm{Cl}(R)$ is an extension of a finite group by $\operatorname{Pic}^{0}(X)$ (cf. [19). Hence the condition $\operatorname{Pic}^{0}(X)$ semi-abelian only depends on $R$. If the reduced exceptional locus of $X$ is a simple normal crossings divisor $E$, then $\operatorname{Pic}^{0}(X)$ is a semi-abelian variety if and only if $\operatorname{Pic}^{0}(X) \cong \operatorname{Pic}^{0}(E)$ (cf. Proposition 5.6). Thus if $\mathcal{L}$ is a line bundle of degree $>2 g-2$ on a nonsingular curve $C$ of genus $g$, then the (completion of the) contraction of the zero section of $\operatorname{Proj}\left(\mathcal{O}_{C} \oplus \mathcal{L}\right)$ is a singularity with class group which is an extension of a finite group by a semi-abelian variety.

To prove Theorem 6.6 we make use of Lang's conjecture (proven by McQuillan [21]), which states that if $H$ is a finitely generated subgroup of a semi-abelian variety $G$ and $Y$ is an irreducible subvariety of $G$ such that $Y \cap H$ is Zariski dense in $Y$, then $Y$ is a translation of a semi-abelian subvariety of $G$. Theorem 8 of $[9]$ (which generalizes to prove the $r=2$ case of Theorem 6.6) uses a very general form of Lang's conjecture for cyclic subgroups of a characteristic 0 algebraic group. This is proven in Theorem 7 of $[9]$.

In Theorem 3.1 we show that topological information on the singularity can be extracted from the series $g$. In fact, the intersection matrix of the exceptional curves of the resolution $X \rightarrow \operatorname{spec}(R)$ as well as $h^{1}\left(X, \mathcal{O}_{X}\right)$ can be extracted from $g$. This result could be compared with the result that the Alexander polynomial of a curve singularity can be recovered from a corresponding series [5]. A related series is shown to be rational for rational surface singularities $\left(H^{1}\left(X, \mathcal{O}_{X}\right)=0\right)$ in [6].

An interesting remaining question when $\mathbf{k}=R / m$ algebraically closed of characteristic zero is if there is a good necessary and sufficient condition on $\mathrm{Cl}(R)$ for the existence of a resolution $X \rightarrow \operatorname{spec}(R)$ such that the series $g$ is irrational. This 
question can be compared with the characterizations of surface singularities with torsion divisor class group in [8].

If $\mathbf{k}$ is a field, and $f \in \mathbf{k}\left[\left[t_{1}, \ldots, t_{r}\right]\right]$ is a formal power series in the variables $t_{1}, \ldots, t_{r}$, we will say that $f$ is rational if there exist polynomials $P, Q \in \mathbf{k}\left[t_{1}, \ldots, t_{r}\right]$ such that $f=\frac{P}{Q}$.

\section{The Riemann-Roch formula for high multiples of a Divisor} ON THE RESOLUTION OF A SURFACE SINGULARITY

This section is a summary of some of the results of section 8 in [9]. Suppose that $R$ is an excellent, normal local ring of dimension 2 , with maximal ideal $m$. Let

$$
f: X \rightarrow \operatorname{spec}(R)
$$

be a resolution of singularities, with integral exceptional divisors $E_{1}, \ldots, E_{r}$.

If $\mathcal{L}$ is a line bundle (or a divisor $D$ ) on $X$ and $C$ is an integral curve on $X$, then $\mathcal{L} \cdot C$ (or $D \cdot C$ ) will denote the line bundle $\mathcal{L} \otimes \mathcal{O}_{C}$ (or the linear equivalence class of $\left.\mathcal{O}_{C} \otimes \mathcal{O}_{X}(D)\right)$. $(\mathcal{L} \cdot C)$ (or $(C \cdot D)$ ) will denote the degree of $\mathcal{L} \otimes \mathcal{O}_{C}$ (or of $\left.\mathcal{O}_{X}(D) \otimes \mathcal{O}_{C}\right)$.

If $\mathcal{M}$ is a coherent sheaf on $X$, then $H^{1}(X, \mathcal{M})$ has finite length as an $R$-module. We will denote

$$
h^{1}(X, \mathcal{M})=\ell\left(H^{1}(X, \mathcal{M})\right) .
$$

The intersection matrix $\left(E_{i} \cdot E_{j}\right)_{1 \leq i, j \leq r}$ is negative definite (section 1 of [23], Lemma 14.1 of [19]).

Let us consider the lattice $\mathbf{E}:=\bigoplus_{i=1}^{r} \mathbf{Z} E_{i}$ and the semigroup

$$
\mathbf{E}^{+}:=\left\{D \in \mathbf{E} \mid \mathcal{O}_{X}(-D) \text { is nef, i.e. }\left(D \cdot E_{i}\right) \leq 0 \text { for } 1 \leq i \leq r\right\} .
$$

Let $\mathbf{E}_{\mathbf{Q}}:=\bigoplus_{i=1}^{r} \mathbf{Q} E_{i}$, and let $\mathbf{E}_{\mathbf{Q}}^{+}$be the rational convex polyhedral cone in $\mathbf{E}_{\mathbf{Q}}$ generated by $\mathbf{E}^{+}$, i.e. $\mathbf{E}_{\mathbf{Q}}^{+}=\bigoplus_{D \in \mathbf{E}^{+}} \mathbf{Q}_{\geq 0} D$. Then $\mathbf{E}_{\mathbf{Q}}^{+}$is a cone contained in $\bigoplus_{i=1}^{r} \mathbf{Q}_{\geq 0} E_{i}\left([19]\right.$, p. 238). Therefore, it is a strongly convex cone, i.e. $\mathbf{E}_{\mathbf{Q}}^{+} \cap$ $\left(-\mathbf{E}_{\mathbf{Q}}^{+}\right)=\{0\}$.

Let $Y=\operatorname{spec}(R)$. Suppose that $D=\sum a_{i} E_{i}$ is a divisor with exceptional support. Then

$$
H^{0}\left(X, \mathcal{O}_{X}(-D)\right) \subset H^{0}\left(\operatorname{spec}(R)-\{m\}, \mathcal{O}_{Y}\right)=R
$$

(since $R$ is normal of dimension 2 ) is an $m$-primary ideal. In fact,

$$
H^{0}\left(X, \mathcal{O}_{X}(-D)\right)=H^{0}\left(X, \mathcal{O}_{X}\left(-\sum b_{i} E_{i}\right)\right),
$$

where $b_{i}=\max \left\{0, a_{i}\right\}$.

Proposition 2.1 ([30], Theorem 7.7). There exists a unique effective $\mathbf{Q}$-divisor $B=\sum b_{i} E_{i}$ such that

(i) $\Delta=D+B$ is in $\mathbf{E}_{\mathbf{Q}}^{+}$, that is $\left(\Delta \cdot E_{i}\right) \leq 0$ for $1 \leq i \leq r$, and

(ii) $\left(\Delta \cdot E_{i}\right)=0$ if $E_{i}$ is a component of $B$.

We will call $\Delta$ the Zariski $\mathbf{Q}$-divisor associated to $D$.

Proposition 2.2 ([7], Proposition 1). Among the divisors $D^{\prime} \in \mathbf{E}^{+}$such that $D^{\prime} \geq$ $D$ there is a minimal one $\bar{D}$. It can be computed by applying the following algorithm: Let $\hat{D}_{1}:=D$ and, for $i \geq 1$, let $\bar{D}:=\hat{D}_{i}$ if $\hat{D}_{i} \in \mathbf{E}^{+}$, or else $\hat{D}_{i+1}:=\hat{D}_{i}+E_{j_{i}}$ where $E_{j_{i}}$ is such that $\left(\hat{D}_{i} \cdot E_{j_{i}}\right)>0$. 
We will call $\bar{D}$ the Laufer divisor associated to $D$, since the previous algorithm is a generalization of Laufer's construction of the fundamental cycle ([17], Prop. 4.1). Note that $\bar{D}$ is the unique divisor in $\mathbf{E}^{+}$such that

$$
H^{0}\left(X, \mathcal{O}_{X}(-D)\right)=H^{0}\left(X, \mathcal{O}_{X}(-\bar{D})\right) \text {. }
$$

Given two Q-divisors $D_{1}, D_{2}$, let us write $D_{1} \leq D_{2}$ if $D_{2}-D_{1}$ is effective.

Lemma 2.3. The following hold:

(i) $D \leq \Delta \leq \bar{D}$.

(ii) For $n \in \mathbf{N}, n \Delta$ is the Zariski $\mathbf{Q}$-divisor associated to $n D$.

(iii) Choose an integer s such that $s \Delta$ is an integral divisor. Suppose that $n$ is a natural number, and $n=a s+b$ with $0 \leq b<s$. Then the natural inclusion

$$
\mathcal{O}_{X}(-a s \Delta-b D) \rightarrow \mathcal{O}_{X}(-n D)
$$

induces an isomorphism of global sections

$$
H^{0}\left(X, \mathcal{O}_{X}(-a s \Delta-b D)\right) \cong H^{0}\left(X, \mathcal{O}_{X}(-n D)\right) .
$$

Proof. The first inequality in (i) holds since the Q-divisor $B$ in Proposition 2.1 is effective. The second one follows from [30, Corollary 7.2, since $\bar{D}-D$ is effective and, for any $E_{i} \in \operatorname{Supp} B$,

$$
\left(((\bar{D}-D)-B) \cdot E_{i}\right)=\left(\bar{D} \cdot E_{i}\right) \leq 0 .
$$

For (ii), note that $n B$ satisfies (i) and (ii) in Proposition 2.1 for $n D$.

For (iii), we have $n D \leq$ as $\Delta+b D \leq n \Delta \leq \overline{n D}$. Hence

$$
H^{0}\left(X, \mathcal{O}_{X}(-\overline{n D})\right) \subseteq H^{0}\left(X, \mathcal{O}_{X}(-a s \Delta-b D)\right) \subseteq H^{0}\left(X, \mathcal{O}_{X}(-n D)\right) .
$$

Since $H^{0}\left(X, \mathcal{O}_{X}(-n D)\right)=H^{0}\left(X, \mathcal{O}_{X}(-\overline{n D})\right)$, we conclude (iii).

Lemma 2.4. Suppose that $m_{1}, \ldots, m_{r} \in \mathbf{Z}$. There exists a constant $c$ such that if $\mathcal{L}$ is a line bundle on $X$ with $\left(\mathcal{L} \cdot E_{i}\right) \geq m_{i}$ for $1 \leq i \leq r$, then

$$
h^{1}(X, \mathcal{L}) \leq c .
$$

Proof. Let $A$ be an effective divisor on $X$ with exceptional support such that $-A$ is ample. There exist $s>0$, a function

$$
\sigma:\{1, \ldots, s\} \rightarrow\{1, \ldots, r\}
$$

and a sequence of divisors $F_{i}, 1 \leq i \leq s$, such that $F_{0}=0, F_{i}=F_{i-1}+E_{\sigma(i)}$ for $1 \leq i \leq s$, and $A=F_{s}$. We have exact sequences

$$
0 \rightarrow \mathcal{O}_{E_{\sigma(i+1)}}\left(-F_{i}\right) \rightarrow \mathcal{O}_{F_{i+1}} \rightarrow \mathcal{O}_{F_{i}} \rightarrow 0
$$

for $1 \leq i \leq s-1$.

Each $E_{i}$ is a locally complete intersection, so by the Riemann-Roch theorem (cf. Exercise IV 1.9 in [14] $\left.H^{1}\left(X, \mathcal{M} \otimes \mathcal{O}_{E_{i}}\right)\right)=0$ if $\mathcal{M}$ is a line bundle on $X$ such that

$$
\left(\mathcal{M} \cdot E_{i}\right)>2 p_{a}\left(E_{i}\right)-2 \text {. }
$$

Thus if $\mathcal{F}$ is a line bundle on $E_{i}$, we have that

$$
h^{0}\left(E_{i}, \mathcal{F}\right)=0 \text { if } \operatorname{deg} \mathcal{F}<0,
$$

and

$$
h^{0}\left(E_{i}, \mathcal{F}\right)=\operatorname{deg} \mathcal{F}+1-p_{a}\left(E_{i}\right) \text { if } \operatorname{deg} \mathcal{F}>2 p_{a}\left(E_{i}\right)-2
$$


We get the further approximation

$$
h^{0}\left(E_{i}, \mathcal{F}\right) \leq p_{a}\left(E_{i}\right) \text { if } 0 \leq \operatorname{deg} \mathcal{F} \leq 2 p_{1}\left(E_{i}\right)-2 .
$$

This can be seen as follows. Let

$$
t=2 p_{a}\left(E_{i}\right)-1-\operatorname{deg} \mathcal{F}>0,
$$

and let $p_{1}, \ldots, p_{t}$ be nonsingular points on $E_{i}$. Let $\mathcal{G}=\mathcal{O}_{E_{i}}\left(p_{1}+\cdots+p_{t}\right)$, a line bundle on $E_{i}$ of degree $t$ with a nonvanishing section. Thus we have an inclusion

$$
H^{0}\left(E_{i}, \mathcal{F}\right) \rightarrow H^{0}\left(E_{i}, \mathcal{F} \otimes \mathcal{G}\right)
$$

and

$$
h^{0}\left(E_{i}, \mathcal{F}\right) \leq h^{0}\left(E_{i}, \mathcal{F} \otimes \mathcal{G}\right)=\left(2 p_{1}\left(E_{i}\right)-1\right)+1-p_{a}\left(E_{i}\right)=p_{a}\left(E_{i}\right) .
$$

We have exact sequences

$$
0 \rightarrow \mathcal{O}_{A}(-n A) \rightarrow \mathcal{O}_{(n+1) A} \rightarrow \mathcal{O}_{n A} \rightarrow 0
$$

for all $n>0$. By (44) there exists $n_{0}$ such that $n \geq n_{0}$ implies

$$
H^{1}\left(X, \mathcal{O}_{A}(-n A) \otimes \mathcal{L}\right)=0
$$

for any line bundle $\mathcal{L}$ on $X$ such that $\left(\mathcal{L} \cdot E_{i}\right) \geq m_{i}$ for $1 \leq i \leq r$. By the formal function theorem,

$$
H^{1}(X, \mathcal{L})=\lim _{\leftarrow} H^{1}\left(X, \mathcal{L} \otimes \mathcal{O}_{n A}\right)=H^{1}\left(X, \mathcal{L} \otimes \mathcal{O}_{n_{0} A}\right) .
$$

Let $\omega_{E_{i}}$ be a canonical divisor on $E_{i}$. Set

$$
\begin{aligned}
d=\max \left\{p_{a}\left(E_{\sigma(1)}\right), \operatorname{deg}\left(\omega_{E_{\sigma(1)}} \otimes \mathcal{O}_{X}\left(F_{0}+\left(n_{0}-1\right) A\right)\right)-m_{\sigma(1)}+1-p_{a}\left(E_{\sigma(1)}\right), \ldots\right. \\
\left.p_{a}\left(E_{\sigma(s)}\right), \operatorname{deg}\left(\omega_{E_{\sigma(s)}} \otimes \mathcal{O}_{X}\left(F_{s-1}+\left(n_{0}-1\right) A\right)\right)-m_{\sigma(s)}+1-p_{a}\left(E_{\sigma(s)}\right)\right\} . \\
h^{1}(X, \mathcal{L})=h^{1}\left(X, \mathcal{L} \otimes \mathcal{O}_{n_{0} A}\right) \\
\leq \sum_{j=0}^{n_{0}-1} h^{1}\left(X, \mathcal{O}_{A}(-j A) \otimes \mathcal{L}\right) \\
\leq \sum_{0 \leq j \leq n_{0}-1}\left(\sum_{1 \leq i \leq s} h^{1}\left(X, \mathcal{O}_{E_{\sigma(i)}}\left(-F_{i-1}-j A\right) \otimes \mathcal{L}\right)\right) \\
=\sum_{0 \leq j \leq n_{0}-1}\left(\sum_{1 \leq i \leq s} h^{0}\left(X, \mathcal{O}_{X}\left(F_{i-1}+j A\right) \otimes \mathcal{L}^{-1} \otimes \omega_{E_{\sigma(i)}}\right)\right) \\
\leq n_{0} s d
\end{aligned}
$$

Lemma 2.5. For fixed $b, 0 \leq b<s$, the function

$$
\sigma_{b}(a)=h^{1}\left(X, \mathcal{O}_{X}(-a s \Delta-b D)\right)
$$

is bounded.

Proof. This is immediate from Lemma 2.4. 
We recall the "local" Riemann-Roch theorem, proved in Lemma 23.1 of [19] as well as in [15]:

(6)

$$
\ell\left(R / H^{0}\left(X, \mathcal{O}_{X}(-D)\right)=-\frac{1}{2}\left(\left(K_{X} \cdot D\right)+\left(D^{2}\right)\right)+h^{1}\left(X, \mathcal{O}_{X}\right)-h^{1}\left(X, \mathcal{O}_{X}(-D)\right),\right.
$$

where $K_{X}$ is a canonical divisor of $X$. For $n=a s+b, 0 \leq b<s$ and $a \geq 0$, let us replace $D$ by $a s \Delta+b D$ in (6), apply Lemma 2.3 (iii), take $a s=n-b$, and use the identity $(\Delta \cdot D)=\left(\Delta^{2}\right)$, which follows since all components of $\Delta-D$ have intersection number 0 with $\Delta$. Then we have

$$
\begin{aligned}
\ell\left(R / H^{0}\left(X, \mathcal{O}_{X}(-n D)\right)\right)=- & \frac{1}{2}\left(\Delta^{2}\right) n^{2}-\frac{1}{2}\left(\Delta \cdot K_{X}\right) n \\
& +\frac{b^{2}}{2}\left(\Delta^{2}\right)+\frac{b}{2}\left(\Delta \cdot K_{X}\right)-\frac{b^{2}}{2}\left(D^{2}\right)-\frac{b}{2}\left(D \cdot K_{X}\right) \\
& +h^{1}\left(X, \mathcal{O}_{X}\right)-\sigma_{b}(a) .
\end{aligned}
$$

At this point we have obtained the following "local" form of a theorem $((1)$ of "A summary of principal results" [30]) of Zariski for projective surfaces.

Proposition 2.6. Let $R$ be an excellent, 2-dimensional equicharacteristic normal local ring. Let $f: X \rightarrow \operatorname{spec}(R)$ be a resolution of singularities and let $D \neq 0$ be a divisor on $X$ supported on the exceptional divisor. Then there exist a natural number $m$, quadratic polynomials $Q_{i}(n)$ for $1 \leq i \leq m$, and a function $\gamma: \mathbf{N} \rightarrow$ $\{1, \ldots, m\}$ such that

$$
\ell\left(R / H^{0}\left(X, \mathcal{O}_{X}(-n D)\right)=Q_{\gamma(n)}(n)\right.
$$

for $n \in \mathbf{N}$.

A slightly different proof of this proposition appears in 22.

An extremely interesting question is if the function $\sigma$ is eventually periodic. This is the "local" form of the question raised by Zariski ((2) of "a summary of principal results" [30]) for projective surfaces. This question of Zariski (and the corresponding local question above) is completely solved in [9]. We prove ([9], Theorem 9) that the answer to the question is "true" in characteristic 0 or over a finite field, but give examples ([9], Example 5) to show that it is "false" in general in positive characteristic.

Suppose that $-F$ is an ample divisor with exceptional support on $X$. For all $m \geq 0$, there is an exact sequence

$$
0 \rightarrow \mathcal{O}_{F}(-m F) \rightarrow \mathcal{O}_{(m+1) F} \rightarrow \mathcal{O}_{m F} \rightarrow 0 .
$$

There exist constants $c_{i}, 1 \leq i \leq r$, such that if $D^{\prime}$ is a divisor on $X$ with $\left(D^{\prime} \cdot E_{i}\right) \geq$ $c_{i}$ for $1 \leq i \leq r$, then $H^{1}\left(F, \mathcal{O}_{F}\left(D^{\prime}\right)\right)=0$. This follows from the proof of Lemma 2.4 .

Lemma 2.7. (i) There exists a natural number $m_{0}$ such that $m \geq m_{0}$ implies that the natural restriction map

$$
H^{1}\left(X, \mathcal{O}_{X}(-a s \Delta-b D)\right) \rightarrow H^{1}\left(\mathcal{O}_{m F}(-a s \Delta-b D)\right)
$$

is an isomorphism for $0 \leq b<s$ and $a \geq 0$. 
(ii) There exists an effective divisor $C$ on $X$ with exceptional support for $f$ such that $\mathcal{O}_{C}(-\Delta)$ is numerically trivial and the restriction map

$$
H^{1}\left(X, \mathcal{O}_{X}(-a s \Delta-b D)\right) \rightarrow H^{1}\left(C, \mathcal{O}_{C}(-a s \Delta-b D)\right)
$$

is an isomorphism for $0 \leq b<s$ and $a>>0$.

Proof. Since $-\Delta$ is nef and $-F$ is ample, there exists $m_{0}$ such that $m \geq m_{0}$ implies

$$
\left((-m F-a s \Delta-b D) \cdot E_{i}\right) \geq c_{i}
$$

for $0 \leq b<s$ and all $a \geq 0$. From the exact sequence (8) we see that

$$
H^{1}\left(\mathcal{O}_{(m+1) F}(-a s \Delta-b D)\right) \cong H^{1}\left(\mathcal{O}_{m F}(-a s \Delta-b D)\right)
$$

for $m \geq m_{0}$. By the formal implicit function theorem, (i) follows.

Let $m_{0} F=C+C^{\prime}$, where

$$
\text { Supp } C=\left\{E_{i} \mid \Delta \cdot E_{i}=0\right\} \text { and } \operatorname{Supp} C^{\prime}=\left\{E_{i} \mid\left(\Delta \cdot E_{i}\right)<0\right\} .
$$

For $a>>0$ we have $H^{1}\left(X, \mathcal{O}_{C^{\prime}}(-C-a s \Delta-b D)\right)=0$; hence the restriction map

$$
H^{1}\left(X, \mathcal{O}_{m_{0} F}(-a s \Delta-b D)\right) \rightarrow H^{1}\left(X, \mathcal{O}_{C}(-a s \Delta-b D)\right)
$$

is an isomorphism, and (ii) holds.

Theorem 2.8 ([9]). Suppose that $R$ is equicharacteristic and that $R$ has residue characteristic 0 , or $R$ has a finite residue field. Then the functions

$$
\sigma_{b}(a)=h^{1}\left(X, \mathcal{O}_{X}(-a s \Delta-b D)\right)
$$

are periodic for $a>>0$.

Proof. When the residue field is finite, it follows as in Theorem 3 of [9]. If $R$ has equicharacteristic 0 , it follows from Theorem 7 of [9] applied to each of the connected components of the Zariski closure in $\operatorname{Pic}^{0}(C)$ of the cyclic group generated by the class of $\mathcal{O}_{C}(-s \Delta)$, where $C$ is the curve of Lemma 2.7 (ii). Theorem 7 in 9 is stated as follows: "Let $G$ be a connected commutative algebraic group defined over an algebraically closed field $\mathbf{k}$ of characteristic 0 , let $x \in G(k)$ and suppose that the cyclic group $\langle x\rangle$ is Zariski dense in $G$. Then, for any subvariety $\Omega \subset G, \Omega \neq G$, the set $\Omega \cap\langle x\rangle$ is finite".

As a consequence of Theorem [2.8 and (17), we have

Theorem 2.9 ([9], Theorem 9). Let $R$ be an excellent, 2-dimensional equicharacteristic normal local ring with a residue field that is either of characteristic zero or a finite field. Let $f: X \rightarrow \operatorname{spec}(R)$ be a resolution of singularities and let $D \neq 0$ be a divisor on $X$ supported on the exceptional divisor. Then there are a quadratic polynomial $Q(n)$ with coefficients in $\mathbf{Q}$ and a periodic function $\lambda: \mathbf{N} \rightarrow \mathbf{Q}$ such that

$$
\ell\left(R / H^{0}\left(X, \mathcal{O}_{X}(-n D)\right)=Q(n)+\lambda(n) .\right.
$$

for all $n$ sufficiently large.

Therefore,

$$
\sum_{n \in \mathbf{N}} \ell\left(R / H^{0}\left(X, \mathcal{O}_{X}(-n D)\right) t^{n}\right.
$$

is a rational series, i.e. a quotient of two polynomials. 


\section{A Hilbert function of $R$}

Let notation be as in the previous section. For $\underline{n}:=\left(n_{1}, \ldots, n_{r}\right) \in \mathbf{Z}^{r}$, set $D_{\underline{n}}:=\sum_{i=1}^{r} n_{i} E_{i}$, and let

$$
R^{*}:=\bigoplus_{\underline{n} \in \mathbf{N}^{r}} H^{0}\left(X, \mathcal{O}_{X}\left(-D_{\underline{n}}\right)\right)
$$

For $\underline{n} \in \mathbf{N}^{r}$, let

$$
h(\underline{n}):=\ell\left(R / H^{0}\left(X, \mathcal{O}_{X}\left(-D_{\underline{n}}\right)\right)\right) .
$$

$h$ can be viewed as a Hilbert-Samuel function of the generally non-Noetherian ring $R^{*}$. It contains a lot of information about the resolution $f: X \rightarrow \operatorname{spec}(R)$, as we will show.

Then

Given $\underline{m}=\left(m_{1}, \ldots, m_{r}\right) \in \mathbf{Z}^{r}$, let $Y=\operatorname{spec}(R), I_{\underline{m}}=H^{0}\left(X, \mathcal{O}_{X}\left(-D_{\underline{m}}\right)\right)$.

$$
I_{\underline{m}} \subset H^{0}\left(Y-\{m\}, \mathcal{O}_{Y}\right)=R,
$$

since $R$ is normal of dimension 2. Also,

$$
\begin{aligned}
I_{\underline{m}}=H^{0}\left(X, \mathcal{O}_{X}\left(-D_{\underline{m}}\right)\right) & =\left\{f \in R \mid \nu_{i}(f) \geq m_{i}, 1 \leq i \leq r\right\} \\
& =\left\{f \in R \mid \nu_{i}(f) \geq \max \left(0, m_{i}\right), 1 \leq i \leq r\right\} .
\end{aligned}
$$

We will prove the following theorem in this section.

Theorem 3.1. From the function $h(\underline{n})$ we can recover the intersection matrix $\left(E_{i} \cdot E_{j}\right)_{1 \leq i, j \leq r}$ and the arithmetic genus $h^{1}\left(X, \mathcal{O}_{X}\right)$ of $X$ and of the $E_{i}, 1 \leq i \leq r$.

Remark. If $R$ has a rational surface singularity, then the converse to Theorem 3.1 is true, i.e. the intersection matrix $\left(E_{i} \cdot E_{j}\right)_{1 \leq i, j \leq r}$ is data equivalent to the function $h(\underline{n})$. In fact, knowing the intersection matrix, for any $\underline{n} \in \mathbf{N}^{r}$, we can determine the Laufer divisor $\overline{D_{\underline{n}}}$ associated to $D_{\underline{n}}$. Then, $H^{1}\left(X, \mathcal{O}_{X}\left(-\overline{D_{\underline{n}}}\right)\right)=0$; thus

$$
h(\underline{n})=-\frac{1}{2}\left(\left(\overline{D_{\underline{n}}} \cdot K_{X}\right)+\left({\overline{D_{\underline{n}}}}^{2}\right)\right)
$$

by (3), and $\left(\bar{D}_{\underline{n}} \cdot K_{X}\right)$ is known since $p_{a}\left(E_{i}\right)=0$ for all $i$.

We now prove Theorem 3.1 .

For $1 \leq i \leq r$, let $R_{i} \subset \mathbf{E}_{\mathbf{Q}}^{+}$be the ray of solutions $v$ to the equations

$$
\left(v \cdot E_{j}\right)=0 \text { for } i \neq j
$$

and

$$
\left(v \cdot E_{i}\right) \leq 0 .
$$

Since $\left(E_{i} \cdot E_{j}\right)$ is negative definite, if $v \in R_{i}$, then $v$ is an effective $\mathbf{Q}$-divisor,

$$
\mathbf{E}_{\mathbf{Q}}^{+}=R_{1}+\cdots+R_{r} .
$$

Let $e_{i} \in \mathbf{Z}^{r}$ be the vector which is 1 in the $i$ th coefficent and 0 in all other coefficients.

Lemma 3.2. Suppose that $\underline{n} \in \mathbf{N}^{r}$. $D_{\underline{n}} \in \mathbf{E}$ is such that $-D_{\underline{n}}$ not nef if and only if there exist $s \in \mathbf{N}$ and $i \in\{1, \ldots, r\}$ such that

$$
h\left(m\left(s \underline{n}+e_{i}\right)\right)=h(m s \underline{n})
$$

for all $m>0$. 
Proof. Let $B_{n}$ be the effective divisor which is the fixed component of the linear system associated to $H^{0}\left(X, \mathcal{O}_{X}(-n D)\right)$. If $D \in \mathbf{E}$ is such that $-D$ is nef, then the set of divisors $B_{n}$ is bounded from above (as in Theorem 10.1 of [30]). Thus the condition (12) cannot occur. If $\underline{n} \in \mathbf{N}^{r}$ and $-D_{\underline{n}}$ is not nef, then there is a Zariski decomposition $\Delta=D_{\underline{n}}+B$ with $B \neq 0$. Choose $s \in \mathbf{N}$ so that $s \Delta$ is integral, and $i$ such that $E_{i}$ is in the support of $B$. (12) now follows from Lemma 2.3.

Lemma 3.3. The rays $R_{i}$ of (11) are determined by the function $h$.

Proof. The anti-nef divisors $\mathbf{E}^{+}$in $\mathbf{E}_{\mathbf{Q}}$ can be determined from $h$ by Lemma 3.2 The cone $\mathbf{E}_{\mathbf{Q}}^{+}$is thus determined by the function $h$, and thus the set of edges $S=\left\{R_{1}, \ldots, R_{r}\right\}$ of $\mathbf{E}_{\mathbf{Q}}^{+}$is determined by $h$. The individual edges $R_{i}$ can now be distinguished by $h$, by Lemma 3.2 and the fact that if $0 \neq \Delta$ is in an edge of $\mathbf{E}_{\mathbf{Q}}^{+}$, then $\Delta$ is in a particular ray $R_{i}$ precisely when there exists $\epsilon>0$ such that $-\Delta+\epsilon E_{i}$ is nef.

Choose $0 \neq \Lambda_{i} \in R_{i}$. There exist (known) $a_{i j} \in \mathbf{Q}$ such that

$$
\Lambda_{i}=\sum a_{i j} E_{j}
$$

for $1 \leq i \leq r$. Let $\left(b_{i j}\right)=\left(a_{i j}\right)^{-1}$. We then have

$$
E_{i}=\sum b_{i j} \Lambda_{j}
$$

From (7) and Lemma 2.5] we can determine $\left(\Lambda_{j} \cdot K_{X}\right)$ for $1 \leq j \leq r$. Then from (14) we can calculate $\left(E_{i} \cdot K_{X}\right)$ for $1 \leq i \leq r$.

Now choose integral divisors (with known $c_{k j}>0$ )

$$
H_{k}=-\left(c_{k 1} E_{1}+\cdots+c_{k r} E_{r}\right)
$$

for $1 \leq k \leq r$ so that the $H_{k}$ are linearly independent in $\mathbf{E}_{\mathbf{Q}}$ and the $-H_{k}$ lie in the interior of $\mathbf{E}^{+}$. Let $\underline{c}_{k}=\left(c_{k 1}, \ldots, c_{k r}\right) \in \mathbf{N}^{r}$. Each $H_{k}$ is ample, since $\left(E_{i} \cdot H_{k}\right)>0$ for all $i$. Thus there exists $m_{0}>0$ such that

$$
h^{1}\left(X, \mathcal{O}_{X}\left(m H_{k}\right)\right)=h^{1}\left(X, \mathcal{O}_{X}\left(m H_{k}+E_{i}\right)\right)=0
$$

for $m \geq m_{0}$ and $1 \leq i, k \leq r$.

By ([6), $m \geq m_{0}$ implies $h\left(m \underline{c}_{k}\right), h\left(m \underline{c}_{k}-e_{i}\right)$ are quadratic polynomials in $m$ for $1 \leq i, k \leq r$. Thus

$$
h\left(m \underline{c}_{k}\right)=\frac{1}{2}\left(\left(m H_{k} \cdot K_{X}\right)-\left(m H_{k}^{2}\right)\right)+\ell\left(H^{1}\left(X, \mathcal{O}_{X}\right)\right)
$$

for $m \geq m_{0}$, and we can determine $\left(H_{k} \cdot K_{X}\right)$ :

$$
\begin{aligned}
h\left(m \underline{c}_{k}-e_{i}\right) & =-\frac{1}{2}\left(H_{k}^{2}\right) m^{2}+\left(\left(H_{k} \cdot\left(\frac{1}{2} K_{X}-E_{i}\right)\right) m\right. \\
& +\frac{1}{2}\left(E_{i} \cdot\left(K_{X}-E_{i}\right)\right)+\ell\left(H^{1}\left(X, \mathcal{O}_{X}\right)\right)
\end{aligned}
$$

for $m \geq m_{0}$. We can now determine $\left(H_{k} \cdot E_{i}\right)$ for $1 \leq i, k \leq r$. We can thus determine the matrix

$$
\left(E_{k} \cdot E_{i}\right)=-\left(c_{k j}\right)^{-1}\left(H_{j} \cdot E_{i}\right) .
$$

Now we can recover the arithmetic genus $p_{a}\left(E_{i}\right)$ of each $E_{i}$ from the formula

$$
2 p_{a}\left(E_{i}\right)-2=\left(E_{i}^{2}\right)+\left(E_{i} \cdot K_{X}\right) .
$$

Since $h^{1}\left(X, \mathcal{O}_{X}\right)$ is known, $p_{a}(X)$ is also recovered from $h$, and hence we have proved Theorem 3.1 . 
In this paper we will study the Hilbert-Samuel function $h(\underline{n})$. We will consider the question of giving a result analogous to formula (9) of Theorem 2.9 for the function $h$. See Theorem 6.6 for a precise statement generalizing these results. In particular, we will study when the series

$$
\sum_{\underline{n} \in \mathbf{N}^{r}} h(\underline{n}) \underline{\underline{n}} \underline{\underline{n}}
$$

is rational.

\section{Piecewise linearity of the Zariski Q-Divisor}

AND PIECEWISE PERIODICITY OF ITS DIFFERENCE WITH THE LAUFER DIVISOR

Suppose that $R$ is a complete normal local ring of dimension two, and $f: X \rightarrow$ $\operatorname{spec}(R)$ is a resolution of singularities with integral exceptional divisors $E_{1}, \ldots, E_{r}$. Let $\mathbf{E}^{+}$be the semigroup in (3) and $\mathbf{E}_{\mathbf{Q}}^{+}$the associated cone, which is contained in $\bigoplus_{i=1}^{r} \mathbf{Q}_{\geq 0} E_{i}$. For $1 \leq i \leq r$, let $\Delta_{i} \in \mathbf{E}_{\mathbf{Q}}=\bigoplus_{i=1}^{r} \mathbf{Q} E_{i}$ be defined by the condition $\left(\Delta_{i} \cdot E_{j}\right)=-\delta_{i j}$, so that $\mathbf{E}_{\mathbf{Q}}^{+}=\bigoplus_{i=1}^{r} \mathbf{Q}_{\geq 0} \Delta_{i}$.

Given $\underline{n} \in \mathbf{Q}^{r}$, let $D_{\underline{n}}=\sum_{i=1}^{r} n_{i} E_{i} \in \mathbf{E}_{\mathbf{Q}}$. For $\underline{n} \in \mathbf{N}^{r}$, let $\Delta_{\underline{n}}\left(\operatorname{resp} . \overline{D_{n}}\right)$ be the Zariski Q-divisor (resp. Laufer divisor) associated to $D_{\underline{n}}$.

Definition 4.1. Let $S$ be a subset of $\{1, \ldots, r\}$. For $\underline{n} \in \mathbf{Q}_{\geq 0}^{r}$, let $\Delta_{\underline{n}}^{S}$ be the orthogonal projection of $D_{\underline{n}}$ on $\left\{E_{i}\right\}_{i \in S}^{\perp}$, i.e. $\Delta_{\underline{n}}^{S} \in \bigoplus_{i=1}^{r} \mathbf{Q} E_{i}$ is defined by

$$
\operatorname{Supp}\left(\Delta_{\underline{n}}^{S}-D_{\underline{n}}\right) \subseteq \bigcup_{i \in S} E_{i}, \quad\left(\Delta_{\underline{n}}^{S} \cdot E_{i}\right)=0 \text { for } i \in S .
$$

We define

$$
\sigma_{S}:=\left\{\underline{n} \in \mathbf{Q}_{\geq 0}^{r} \mid \Delta_{\underline{n}}^{S}-D_{\underline{n}} \geq 0, \quad \Delta_{\underline{n}}^{S} \in \mathbf{E}_{\mathbf{Q}}^{+}\right\} .
$$

Let $\mathbf{F}_{S}$ be the face of $\mathbf{E}_{\mathbf{Q}}^{+}$orthogonal to $\left\{E_{i}\right\}_{i \in S}$; that is, $\mathbf{F}_{S}=\bigoplus_{j \notin S} \mathbf{Q}_{\geq 0} \Delta_{j}$. Note that

$$
\sigma_{S}=\left\{\underline{n} \in \mathbf{Q}_{\geq 0}^{r} \mid\left(D_{\underline{n}}+\left(\bigoplus_{i \in S} \mathbf{Q}_{\geq 0} E_{i}\right)\right) \cap \mathbf{F}_{S} \neq \emptyset\right\} .
$$

Given $S \subseteq\{1, \ldots, r\}$, let $S=S_{1} \cup \ldots \cup S_{k}$ be the partition of $S$ determined by the connected components of $\bigcup_{i \in S} E_{i}$, and let

$$
e_{S}=\text { s.c.m. }\left\{\left|\operatorname{det}\left(E_{i} \cdot E_{j}\right)_{i, j \in S_{l}}\right|\right\}_{l=1, \ldots, k} \cdot
$$

Let $J:=\{1, \ldots, r\} \backslash S$.

Theorem 4.2. The following hold:

(i) Given $\underline{n} \in \mathbf{Q}_{\geq 0}^{r}$, let $\underline{n}_{J}=\left(n_{j}\right)_{j \in J}$ be its projection. Then,

$$
\Delta_{\underline{n}}^{S}=\sum_{j \in J} l_{j}^{S}\left(\underline{n}_{J}\right) \Delta_{j}=D_{\underline{n}}+\sum_{i \in S} b_{i}^{S}(\underline{n}) E_{i},
$$

where $l_{j}\left(\underline{n}_{J}\right)$ (resp. $\left.b_{i}^{S}(\underline{n})\right)$ are linear functions on $\underline{n}_{J}$ (resp. $\left.\underline{n}\right)$ with coefficients in $\frac{1}{e_{S}} \mathbf{Z}$.

(ii) For $\underline{n} \in \mathbf{N}^{r}, \underline{n} \in \sigma_{S}$ if and only if $\Delta_{\underline{n}}^{S}$ is the Zariski $\mathbf{Q}$-divisor $\Delta_{\underline{n}}$ associated to $D_{\underline{n}}$.

(iii) For any $S \subset\{1, \ldots, r\}$ (strictly contained), $\sigma_{S}$ is a strongly convex rational polyhedral cone of dimension $r$. The set $\Sigma$ consisting of all $\sigma_{S}$ 's and its faces is a fan subdivision of $\mathbf{Q}_{\geq 0}^{r}$. 
Proof. For $S \subseteq\{1, \ldots, r\}$ and for any $\underline{n} \in \mathbf{Q}_{\geq 0}^{r}$ we have

$$
\Delta_{\underline{n}}^{S}=D_{\underline{n}}+\sum_{i \in S} b_{i}^{S}(\underline{n}) E_{i}
$$

where the $b_{i}^{S}(\underline{n})$ are defined by

$$
\sum_{i \in S} b_{i}^{S}(\underline{n})\left(E_{i} \cdot E_{i^{\prime}}\right)=-\left(D_{\underline{n}} \cdot E_{i^{\prime}}\right) \quad \text { for all } i^{\prime} \in S .
$$

Therefore the $b_{i}^{S}$ are linear functions on $\underline{n}$ with coefficients in $1 / e_{S} \mathbf{Z}$. We may also write

$$
\Delta_{\underline{n}}^{S}=\sum_{j \in J} l_{j}^{S}(\underline{n}) \Delta_{j}
$$

where the $l_{j}^{S}(\underline{n})$ are defined by $\left(\left(\Delta_{\underline{n}}^{S}-D_{\underline{n}}\right) \cdot \Delta_{j^{\prime}}\right)=0$ for $j^{\prime} \in J$, i.e.

$$
\sum_{j \in J} l_{j}^{S}(\underline{n})\left(\Delta_{j} \cdot \Delta_{j^{\prime}}\right)=-n_{j^{\prime}} \quad \text { for all } j^{\prime} \in J
$$

Hence the $l_{j}^{S}$ are linear functions on $\underline{n}_{J}$ with coefficients in $\mathbf{Q}$. Since $\left.l_{j}^{S} \underline{n}_{J}\right)=$ $-\left(\Delta_{\underline{n}}^{S} \cdot E_{j}\right)$, the coefficients are in $\frac{1}{e_{S}} \mathbf{Z}$; hence (i) holds

For (ii), note that $\Delta_{\underline{n}}-D_{\underline{n}}$ effective, $\Delta_{\underline{n}} \in \mathbf{E}_{\mathbf{Q}}^{+}$and $\left(\Delta_{\underline{n}} \cdot E_{i}\right)=0$ for $E_{i} \in$ $\operatorname{Supp}\left(\Delta_{\underline{n}}-D_{\underline{\underline{n}}}\right)$ characterize the Zariski Q-divisor $\Delta_{\underline{n}}$ associated to $D_{\underline{\underline{n}}}$. Hence (ii) follows from (15) and (16).

For (iii), (16) and (18) imply that

$$
\sigma_{S}=\left\{\underline{n} \in \mathbf{Q}_{\geq 0}^{r} \mid b_{i}^{S}(\underline{n}) \geq 0 \text { for } i \in S, \quad l_{j}^{S}\left(\underline{n}_{J}\right) \geq 0 \text { for } j \in J\right\}
$$

Therefore, $\sigma_{S}$ is the intersection of a finite number of rational halfspaces, thus a rational convex polyhedral cone. Since it is contained in $\mathbf{Q}_{>0}^{r}$, it is strongly convex. If $\operatorname{dim} \sigma_{S}<r$, then $\sigma_{S}$ is contained in a hyperplane $H$. Since the face $\mathbf{F}_{S}$ of $\mathbf{E}_{\mathbf{Q}}^{+}$orthogonal to $S$ is contained in $\sigma_{S}, H$ must be the orthogonal of a divisor $D \in \bigoplus_{i \in S} \mathbf{Z} E_{i}$. For $i \in S$, the projection of $\mathbf{F}_{S}$ from $E_{i}$ in $\bigoplus_{j \neq i} \mathbf{Q} E_{j}$ is contained in $\sigma_{S}$, thus in $H$. If $\mathbf{F}_{S} \neq\{0\}$, i.e. $S$ is strictly contained in $\{1, \ldots, r\}$, this implies $\left(D \cdot E_{i}\right)=0$ and, by the negative definiteness of the intersection matrix restricted to $S, D=0$. Therefore $\operatorname{dim} \sigma_{S}=r$.

Let us show that

$$
\bigcup_{S \subseteq\{1, \ldots, r\}} \sigma_{S}=\mathbf{Q}_{\geq 0}^{r} .
$$

The inclusion $\subseteq$ is clear, and, since the $\sigma_{S}$ 's are cones, it suffices to prove that $\mathbf{N}^{r} \subseteq \bigcup_{S}\left(\sigma_{S} \cap \mathbf{N}^{r}\right)$. Let $\underline{n} \in \mathbf{N}^{r}$ and let $S=\left\{i \mid E_{i} \subseteq \operatorname{Supp}\left(\Delta_{\underline{n}}-D_{\underline{n}}\right)\right\}$. Then $\Delta_{\underline{n}}^{S}=\Delta_{\underline{n}}$ by (15), and hence $\underline{n} \in \sigma_{S}$ by (ii).

In order to prove that $\Sigma$ is a fan, it is enough to show that, for $S, S^{\prime} \subset\{1, \ldots, r\}$, $\sigma_{S} \cap \sigma_{S^{\prime}}$ is a face of $\sigma_{S}$. Let $S, S^{\prime} \subset\{1, \ldots, r\}$; then

$$
\sigma_{S} \cap \sigma_{S^{\prime}}=\left\{\underline{n} \in \mathbf{Q}_{\geq 0}^{r} \mid \Delta_{\underline{n}}^{S}=\Delta_{\underline{n}}^{S^{\prime}} \geq D_{\underline{n}}, \Delta_{\underline{n}}^{S} \in \mathbf{E}_{\mathbf{Q}}^{+}\right\}
$$


In fact, from (ii) follows the equality of both members intersected with $\mathbf{N}^{r}$. Since they are both cones by (i), and contained in $\mathbf{Q}_{\geq 0}^{r}$, we conclude the equality. Therefore,

$$
\begin{array}{r}
\sigma_{S} \cap \sigma_{S^{\prime}}=\sigma_{S} \cap\left\{\underline{n} \in \mathbf{Q}_{\geq 0}^{r} \mid b_{i}^{S}(\underline{n})=0 \text { for } i \in S \backslash\left(S^{\prime} \cap S\right),\right. \\
\left.l_{j}^{S}\left(\underline{n}_{J}\right)=0 \quad \text { for } j \in S^{\prime} \backslash\left(S \cap S^{\prime}\right)\right\}
\end{array}
$$

is a face of $\sigma_{S}$ by (19).

Corollary 4.3. The fan $\Sigma$ consisting of the cones $\sigma_{S}$ in Definition 4.1 and its faces satisfies the following property: For each $\sigma \in \Sigma$, each of the coefficients of the Zariski Q-divisor, that is, of the function

$$
\sigma \cap \mathbf{Z}^{r} \rightarrow \mathbf{E}_{\mathbf{Q}} \cong \mathbf{Q}^{r}, \quad \underline{n} \mapsto \Delta_{\underline{n}},
$$

is a linear function of $\underline{n}$ with coefficients in $\mathbf{Q}$. Moreover, if $\sigma \subseteq \sigma_{S}$, then the coefficients are in $\frac{1}{e_{S}} \mathbf{N}$.

Next we will subdivide the fan $\Sigma$ in order to have a certain periodicity for the coefficients of the function $\underline{n} \mapsto \overline{D_{\underline{n}}}-\Delta_{\underline{n}}$ (Theorem 4.9 ). The subdivision we will give consists of rational convex polyhedral sets. By a rational convex polyhedral set in $\mathbf{Q}^{r}$, or more simply a polyhedral set, we mean a set of the form

$$
P=\left\{\underline{n} \in \mathbf{Q}^{r} \mid L_{i}(\underline{n}) \geq b_{i}, \quad 1 \leq i \leq m\right\},
$$

where $m \in \mathbf{N}$ and, for $1 \leq i \leq m, L_{i}$ is an integral linear form on $\mathbf{Q}^{r}$ and $b_{i} \in \mathbf{Z}$. We define the cone associated to $P$ to be

$$
\sigma_{P}:=\left\{\underline{n} \in \mathbf{Q}^{r} \mid L_{i}(\underline{n}) \geq 0, \quad 1 \leq i \leq m\right\} .
$$

A subset $Q$ of $P$ is called a face of $P$ if there exist an integral linear form $L$ on $\mathbf{Q}^{r}$ and $b \in \mathbf{Z}$ such that

$$
P \subseteq\left\{\underline{n} \in \mathbf{Q}^{r} \mid L(\underline{n}) \geq b\right\} \quad \text { and } \quad Q=P \cap\left\{\underline{n} \in \mathbf{Q}^{r} \mid L(\underline{n})=b\right\} .
$$

Definition 4.4. An abstract complex of polyhedral sets in $\mathbf{Q}^{r}$ is a finite set $\mathcal{P}=$ $\left\{P_{\gamma}\right\}_{\gamma \in \Lambda}$ of polyhedral sets in $\mathbf{Q}^{r}$ such that $P$ has dimension $r$ for all $P \in \mathcal{P}$. Given a fan $\Sigma$ in $\mathbf{Q}^{r}$, an abstract complex of polyhedral sets $\mathcal{P}=\left\{P_{\gamma}\right\}_{\gamma \in \Lambda}$ is a subdivision of $\Sigma$ with the same associated cones if

(i) $\bigcup_{\gamma \in \Lambda} P_{\gamma}$ is the support of $\Sigma$, and

(ii) for each $P_{\gamma} \in \mathcal{P}$, there exists $\sigma \in \Sigma$ such that $P_{\gamma} \subseteq \sigma$.

Definition 4.5. Let $S$ be a subset of $\{1, \ldots, r\}$ and $J=\{1, \ldots, r\} \backslash S$. For any pair $(\alpha, \beta)$, where

$$
\alpha: T \rightarrow \frac{1}{e_{S}} \mathbf{N} \quad \beta: J \backslash T \rightarrow \frac{1}{e_{S}} \mathbf{N}
$$

are maps defined on some subset $T$ of $J$ and its complement, we define the polyhedral set in $\mathbf{Q}^{r}$

$$
P_{S}(\alpha, \beta):=\left\{\underline{n} \in \sigma_{S} \mid l_{j}^{S}(\underline{n})=\alpha(j) \text { for } j \in T, \quad l_{j}^{S}(\underline{n}) \geq \beta(j) \text { for } j \in J \backslash T\right\}
$$

where $l_{j}^{S}(\underline{n})=l_{j}^{S}\left(\underline{n}_{J}\right)$ are the linear functions with coefficients in $\frac{1}{e_{S}} \mathbf{Z}$ in Theorem 3.2 (i). We will simply denote $P_{S}(\alpha, \beta)$ by $P(\alpha, \beta)$ if there is no possible confusion on $S$. 
Note that the cone associated to $P(\alpha, \beta)$ is

$$
\sigma_{P(\alpha, \beta)}=\left\{\underline{n} \in \sigma_{S} \mid l_{j}^{S}(\underline{n})=0 \text { for } j \in T\right\}=\sigma_{S} \cap \sigma_{S \cup T},
$$

which is a face of $\sigma_{S}$ (see (20) ).

Lemma 4.6. Let $S$ be a subset of $\{1, \ldots, r\}$ and $J=\{1, \ldots, r\} \backslash S$. Suppose that an assignment

$$
\alpha \mapsto \alpha^{c}
$$

is given to each map $\alpha: T \rightarrow \frac{1}{e_{S}} \mathbf{N}$, defined on some subset $T$ of $J$, of a map $\alpha^{c}: J \backslash T \rightarrow \frac{1}{e_{S}} \mathbf{N}$. Then, there exists an abstract complex of polyhedral sets $\mathcal{P}_{\sigma_{S}}$ subdividing $\sigma_{S}$ with the same associated cones such that

$$
\text { for all } P \in \mathcal{P}_{\sigma_{S}} \text { there exists a map } \alpha \text { such that } P \cap \mathbf{Z}^{r} \subseteq P_{S}\left(\alpha, \alpha^{c}\right) \text {. }
$$

Therefore, if an assignation as before is given for every subset $S$ of $\{1, \ldots, r\}$, then there exists an abstract complex of polyhedral sets $\mathcal{P}$ subdividing the fan $\Sigma$ in Corollary 3.3 with the same associated cones and such that

for all $P \in \mathcal{P}$ there exist a set $S$ and a map $\alpha$ such that $P \cap \mathbf{Z}^{r} \subseteq P_{S}\left(\alpha, \alpha^{c}\right)$.

Proof. The second assertion follows from the first (see the remark before Definition 4.5). To prove the first one, we start by defining a finite set $\Lambda$ of pairs $(\alpha, \beta)$ such that

and

$$
P(\alpha, \beta) \subseteq P\left(\alpha, \alpha^{c}\right)
$$

$$
\sigma_{S} \cap \mathbf{Z}^{r}=\bigcup_{(\alpha, \beta) \in \Lambda}\left(P(\alpha, \beta) \cap \mathbf{Z}^{r}\right),
$$

the union being disjoint.

Let $\Lambda_{0}=\left\{\alpha_{0}\right\}$, where $\alpha_{0}: \emptyset \rightarrow \frac{1}{e_{S}} \mathbf{N}$ is the trivial map, and let $\beta_{0}=\alpha_{0}^{c}$. Let $1 \leq t \leq \sharp J$, and suppose we have defined a finite set $\Lambda_{t-1}$ of maps $\alpha^{\prime}: T^{\prime} \rightarrow \frac{1}{e_{S}} \mathbf{N}$, where $T^{\prime} \subset J$ has cardinal $\leq t-1$, and, for each $\alpha^{\prime} \in \Lambda_{t-1}$, a map $\beta^{\prime}: J \backslash T^{\prime} \rightarrow \frac{1}{e_{S}} \mathbf{N}$, $\beta^{\prime}(j) \geq \alpha^{c}(j)$ for $j \in J \backslash T^{\prime}$, so that $P\left(\alpha^{\prime}, \beta^{\prime}\right) \subseteq P\left(\alpha^{\prime}, \alpha^{\prime c}\right)$. Let $\Lambda_{t}$ be the union of $\Lambda_{t-1}$ and all maps $\alpha: T \rightarrow \frac{1}{e_{S}} \mathbf{N}$, where $\sharp T=t$ and there exists $T^{\prime} \subset T$ with $\sharp T^{\prime}=t-1$, such that $\alpha^{\prime}=\left.\alpha\right|_{T^{\prime}}$ belongs to $\Lambda_{t-1}$ and

$$
\alpha(j)<\beta^{\prime}(j) \text { for } j \in T \backslash T^{\prime} .
$$

For any of these maps $\alpha$, let $\beta: J \backslash T \rightarrow \frac{1}{e_{S}} \mathbf{N}$,

$$
\beta(j)=\sup \left\{\alpha^{c}(j),\left\{\widetilde{\beta}(j) \mid \widetilde{\alpha}=(\alpha \mid \widetilde{T}) \in \Lambda_{t-1}, \quad \widetilde{\alpha}: \widetilde{T} \rightarrow \frac{1}{e_{S}} \mathbf{N}, \quad j \notin \widetilde{T}\right\}\right\} \quad \text { for } j \in J \backslash T
$$

Finally, set $\Lambda:=\left\{(\alpha, \beta) \mid \alpha \in \Lambda_{\sharp J}\right\}$.

Condition (25) implies that $\Lambda_{t}$, and hence $\Lambda$, is a finite set. By (26), we have $\beta(j) \geq \alpha^{c}(j)$ for $j \in J \backslash T$; hence

$$
P(\alpha, \beta) \subseteq P\left(\alpha, \alpha^{c}\right) .
$$

Let us prove (24). First note that, for any $\alpha^{\prime} \in \Lambda_{t-1}, 1 \leq t \leq \sharp J$, we have

$$
P\left(\alpha^{\prime}, 0\right) \cap \mathbf{Z}^{r}=\left(P\left(\alpha^{\prime}, \beta^{\prime}\right) \cap \mathbf{Z}^{r}\right) \cup\left(\bigcup_{\alpha \in \Lambda_{t},\left.\alpha\right|_{T^{\prime}}=\alpha^{\prime}}\left(P(\alpha, 0) \cap \mathbf{Z}^{r}\right)\right) .
$$


In fact, if $\underline{n} \in\left(P\left(\alpha^{\prime}, 0\right) \cap \mathbf{Z}^{r}\right) \backslash\left(P\left(\alpha^{\prime}, \beta^{\prime}\right) \cap \mathbf{Z}^{r}\right)$ then, there exists $j \in J \backslash T^{\prime}$ such that $l_{j}^{S}(\underline{n})<\beta^{\prime}(j)$, and $l_{j}^{S}(\underline{n}) \in \frac{1}{e_{S}} \mathbf{N}$ by (i) in Theorem 4.2, Let $T=T^{\prime} \cup\{j\}$ and $\alpha: T \rightarrow \frac{1}{e_{S}} \mathbf{N}$, defined by $\left.\alpha\right|_{T^{\prime}}=\alpha^{\prime}, \alpha(j)=-\left(\Delta_{\underline{n}} \cdot E_{j}\right)=l_{j}^{S}(\underline{n})$. Then $\alpha$ satisfies (19); thus $\alpha \in \Lambda_{t}$, and $\underline{n} \in P(\alpha, 0)$. Since $\sigma_{S}=P\left(\alpha_{0}, 0\right)$ and for $\alpha \in \Lambda_{\sharp J}$ we have $P(\alpha, \beta)=P(\alpha, 0)$, we conclude 24).

In order to prove that the union is disjoint, let $\left(\alpha_{1}, \beta_{1}\right),\left(\alpha_{2}, \beta_{2}\right) \in \Lambda,\left(\alpha_{1}, \beta_{1}\right) \neq$ $\left(\alpha_{2}, \beta_{2}\right), \alpha_{i}: T_{i} \rightarrow \frac{1}{e_{S}} \mathbf{N}$ for $i=1,2$. If $T_{1}=T_{2}$, then $\alpha_{1} \neq \alpha_{2}$ and $P\left(\alpha_{1}, \beta_{1}\right) \cap$ $P\left(\alpha_{2}, \beta_{2}\right)=\emptyset$. Suppose $T_{1} \neq T_{2}$; we may assume that $T_{1} \not \subset T_{2}$ and that

$$
\alpha_{1}\left|T_{1} \cap T_{2}=\alpha_{2}\right| T_{1} \cap T_{2}
$$

Let

$$
T_{1}=T^{0} \supset T^{1} \supset \cdots \supset T^{i} \supset T^{i+1} \supset \cdots \supset T^{k+1}
$$

be such that $\alpha^{i}:=\left.\alpha_{1}\right|_{T^{i}} \in \Lambda_{t_{1}-i}$ where $t_{1}=\sharp T_{1}, T^{i}=T^{i+1} \cup\left\{j_{i}\right\}, \alpha_{1}\left(j_{i}\right)=$ $\alpha^{i}\left(j_{i}\right)<\beta^{i+1}\left(j_{i}\right)$ and $T^{k+1} \subset T_{1} \cap T_{2}, T^{k} \not \subset T_{1} \cap T_{2}$. Then $k \geq 0, j_{k} \notin T_{2}$, and

$$
\alpha_{1}\left(j_{k}\right)<\beta^{k+1}\left(j_{k}\right) \text {. }
$$

If $P\left(\alpha_{1}, \beta_{1}\right) \cap P\left(\alpha_{2}, \beta_{2}\right) \neq \emptyset$, then $\alpha_{1}\left(j_{k}\right) \geq \beta_{2}\left(j_{k}\right)$; but, by (26),$\beta_{2}\left(j_{k}\right) \geq \beta^{k+1}\left(j_{k}\right)$. Therefore $P\left(\alpha_{1}, \beta_{1}\right) \cap P\left(\alpha_{2}, \beta_{2}\right)=\emptyset$.

Now, for any $(\alpha, \beta) \in \Lambda$, let us consider the polyhedral set

$$
\begin{gathered}
\bar{P}(\alpha, \beta)=\left\{\underline{n} \in \sigma_{S} \mid \alpha(j)-\frac{1}{2 e_{S}} \leq l_{j}^{S}(\underline{n}) \leq \alpha(j)+\frac{1}{2 e_{S}} \text { for } j \in T,\right. \\
\left.\beta(j)-\frac{1}{2 e_{S}} \leq l_{j}^{S}(\underline{n}) \text { for } j \in J \backslash T\right\},
\end{gathered}
$$

whose associated cone is $\sigma_{P(\alpha, \beta)}=\sigma_{S} \cap \sigma_{S \cup T}$. We have:

(a) $P(\alpha, \beta) \cap \mathbf{Z}^{r}=\bar{P}(\alpha, \beta) \cap \mathbf{Z}^{r}$, and

(b) $\sigma_{S}=\bigcup_{(\alpha, \beta) \in \Lambda} \bar{P}(\alpha, \beta)$.

This implies that the set $\{\bar{P}(\alpha, \beta)\}_{(\alpha, \beta) \in \Lambda}$ is an abstract complex of polyhedral sets $\mathcal{P}_{\sigma_{S}}$ subdividing $\sigma_{S}$ with the same associated cones and satisfying (23).

For every subset $S$ of $\{1, \ldots, r\}$, let us construct an assignment $\alpha \mapsto \alpha^{c}$ as in Lemma 4.6] such that the function $\underline{n} \mapsto \overline{D_{\underline{n}}}-\Delta_{\underline{n}}$ has good properties on the sets $P_{S}\left(\alpha, \alpha^{c}\right)$.

Let us fix $S$ and $T \subseteq J=\{1, \ldots, r\} \backslash S$. In a similar way as in Proposition 2.2 given a divisor $D=\sum_{i=1}^{r} n_{i} E_{i}$, among the divisors $D^{\prime} \geq D$ such that $\left(D^{\prime} \cdot E_{i}\right) \leq 0$ for all $i \in S \cup T$, there is a minimal one. Let us denote it by $\tilde{D}^{(S \cup T)}$, or $\widetilde{D}$ if there is no possible confusion on $S \cup T$. For $\underline{n} \in \sigma_{S}$, we have

$$
\overline{D_{\underline{n}}} \geq \tilde{D_{\underline{n}}} \geq \Delta_{\underline{n}}
$$

and $\widetilde{D_{\underline{n}}}$ may be computed as follows: Let $\hat{D}_{1}:=\left\lceil\Delta_{\underline{n}}\right\rceil$, where, if $\Delta_{\underline{n}}=\sum_{i=1}^{r} q_{i} E_{i}$, $q_{i} \in \mathbf{Q}_{\geq 0}$, then $\left\lceil\Delta_{\underline{n}}\right\rceil=\sum_{i=1}^{r}\left\lceil q_{i}\right\rceil E_{i}$. For $i \geq 1$, let $\widetilde{D}_{D}:=\hat{D}_{i}$ if $\left(\hat{D}_{i} \cdot E_{j}\right) \leq 0$ for all $j \in S \cup T$, or else $\hat{D}_{i+1}:=\hat{D}_{i}+E_{j_{i}}$, where $j_{i} \in S \cup T$ is such that $\left(\hat{D}_{i} \cdot E_{j_{i}}\right)>0$.

Given a map $\alpha: T \rightarrow \frac{1}{e_{S}} \mathbf{N}$, let $P(\alpha, 0)$ be the polyhedral set defined by (22) for the map $\beta$ identically 0 . Note that $P(\alpha, 0) \supseteq P(\alpha, \beta)$ for any other map $\beta$. 
Lemma 4.7. For any map $\alpha: T \rightarrow \frac{1}{e_{S}} \mathbf{N}$, if $\underline{n}, \underline{m} \in P(\alpha, 0) \cap \mathbf{Z}^{r}$ and $\left\lceil\Delta_{\underline{n}}\right\rceil-\Delta_{\underline{n}}=$ $\left\lceil\Delta_{\underline{m}}\right\rceil-\Delta_{\underline{m}}$ (for example if $\underline{n}-\underline{m} \in e_{S} \mathbf{Z}^{r}$ ), then

$$
\tilde{D_{\underline{n}}}-\Delta_{\underline{n}}=\tilde{D_{\underline{m}}}-\Delta_{\underline{m}} .
$$

Proof. Let $\hat{D}_{1}=\left\lceil\Delta_{\underline{n}}\right\rceil<\hat{D_{2}}<\cdots<\hat{D}_{k}=\tilde{D_{\underline{n}}}$ be defined as above. Let us show by induction on $i$ that $\hat{\hat{D}}_{i} \leq \tilde{D_{\underline{m}}}+\left(\Delta_{\underline{n}}-\Delta_{\underline{m}}\right)$; hence $\tilde{D_{\underline{n}}}-\Delta_{\underline{n}} \leq \tilde{D_{\underline{m}}}-\Delta_{\underline{m}}$ and, in an analogous way, we obtain the other inequality.

For $i=1, \hat{D_{1}}=\left\lceil\Delta_{\underline{n}}\right\rceil=\left\lceil\Delta_{\underline{m}}\right\rceil+\left(\Delta_{\underline{n}}-\Delta_{\underline{m}}\right) \leq \tilde{D_{\underline{m}}}+\left(\Delta_{\underline{n}}-\Delta_{\underline{m}}\right)$. Suppose $\hat{D}_{i} \leq \tilde{D_{\underline{m}}}+\left(\Delta_{\underline{n}}-\Delta_{\underline{m}}\right)$. Let $\hat{D}_{i+1}=\hat{D}_{i}+E_{j_{i}}$, where $j_{i} \in S \cup T,\left(\hat{D}_{i} \cdot E_{j_{i}}\right)>0$. If $j_{i} \in \bar{S}$, then $\left(\Delta_{\underline{n}} \cdot E_{j_{i}}\right)=\left(\Delta_{\underline{m}} \cdot E_{j_{i}}\right)=0$; and, if $j_{i} \in T$, then $\left(\Delta_{\underline{n}} \cdot E_{j_{i}}\right)=$ $\left(\Delta_{\underline{m}} \cdot E_{j_{i}}\right)=-\alpha\left(E_{j_{i}}\right)$. Therefore

$$
\left(\left(\tilde{D_{\underline{m}}}+\left(\Delta_{\underline{n}}-\Delta_{\underline{m}}\right)-\hat{D_{i}}\right) \cdot E_{j_{i}}\right)=\left(\tilde{\tilde{D_{\underline{m}}}} \cdot E_{j_{i}}\right)-\left(\hat{D}_{i} \cdot E_{j_{i}}\right)<0 .
$$

Since $\Delta_{\underline{n}}-\Delta_{\underline{m}} \in \mathbf{E}$, this implies that $E_{j_{i}} \leq \tilde{D_{\underline{m}}}+\left(\Delta_{\underline{n}}-\Delta_{\underline{m}}\right)-\hat{D}_{i}$, that is, $\hat{D_{i+1}} \leq \hat{D_{\underline{m}}}+\left(\Delta_{\underline{n}}-\Delta_{\underline{m}}\right)$.

Lemma 4.8. For any subset $T$ of $J$ and any map $\alpha: T \rightarrow \frac{1}{e_{S}} \mathbf{N}$, there exists a map $\alpha^{c}: J \backslash T \rightarrow \frac{1}{e_{S}} \mathbf{N}$ such that

$$
\overline{D_{\underline{n}}}=\stackrel{\sim}{D}^{(S \cup T)} \quad \text { for } \underline{n} \in P\left(\alpha, \alpha^{c}\right) \cap \mathbf{Z}^{r} .
$$

Therefore

$$
\overline{D_{\underline{n}}}-\Delta_{\underline{n}}=\overline{D_{\underline{m}}}-\Delta_{\underline{m}} \quad \text { for } \underline{n}, \underline{m} \in P\left(\alpha, \alpha^{c}\right) \cap \mathbf{Z}^{r}, \underline{n}-\underline{m} \in e_{S} \mathbf{Z}^{r} .
$$

Proof. There exists $\left\{\underline{n}_{1}, \cdots, \underline{n}_{t}\right\} \subset P(\alpha, 0) \cap \mathbf{Z}^{r}$ such that

$$
P(\alpha, 0) \cap \mathbf{Z}^{r} \subset \bigcup_{i=1}^{t}\left(\underline{n}_{i}+e_{S} \mathbf{Z}^{r}\right) .
$$

For $j \in J \backslash T$, let

$$
\alpha^{c}(j)=\sup \left\{0,\left(\left(\tilde{D}_{\underline{n}_{i}}-\Delta_{\underline{n}_{i}}\right) \cdot E_{j}\right), 1 \leq i \leq t\right\} .
$$

By the previous lemma, if $\underline{n} \in P(\alpha, 0) \cap \mathbf{Z}^{r}$, then there exists $i, 1 \leq i \leq t$, such that $\widetilde{D}_{\underline{n}}-\Delta_{\underline{n}}=\widetilde{D}_{\underline{n}_{i}}-\Delta_{\underline{n}_{i}}$. Therefore, if $\underline{n} \in P\left(\alpha, \alpha^{c}\right)$ then $\left(\widetilde{\widetilde{D}}_{\underline{n}} \cdot E_{j}\right) \leq 0$ for all $j \in J \backslash T$, and hence $\bar{D}_{\underline{n}}=\widetilde{D}_{\underline{n}}$. The second assertion follows again from Lemma 4.7

From Lemmas $4.6,4.7$ and 4.8 we conclude

Theorem 4.9. There exists an abstract complex of polyhedral sets $\mathcal{P}$ subdividing the fan $\Sigma$ in Corollary 4.3, with the same associated cones, such that, for every $P \in \mathcal{P}$, if $P \subseteq \sigma_{S}$ then

$$
\overline{D_{\underline{n}}}-\Delta_{\underline{n}}=\overline{D_{\underline{m}}}-\Delta_{\underline{m}} \quad \text { for } \underline{n}, \underline{m} \in P \cap \mathbf{Z}^{r}, \underline{n}-\underline{m} \in e_{S} \mathbf{Z}^{r} .
$$


Example 4.10. In an $A_{4}$-singularity, whose dual graph is

$$
E_{1} \quad E_{2} \quad E_{3} \quad E_{4}
$$

and all self-intersections are -2 , let us consider $\sigma_{\{2,4\}}$, which is given by

$$
n_{2} \leq \frac{n_{1}+n_{3}}{2}, \quad n_{4} \leq \frac{n_{3}}{2}, \quad \frac{n_{1}}{2} \leq n_{3} \leq 3 n_{1} .
$$

For $\underline{n} \in \sigma_{\{2,4\}} \cap \mathbf{Z}^{4}$,

$$
\Delta_{\underline{n}}=n_{1} E_{1}+\frac{n_{1}+n_{3}}{2} E_{2}+n_{3} E_{3}+\frac{n_{3}}{2} E_{4} .
$$

Let $P_{0}:=\sigma_{\{2,4\}} \cap\left\{\underline{n} \mid\left(\Delta_{\underline{n}} \cdot E_{3}\right)=0\right\}$ and $P_{1}=\sigma_{\{2,4\}} \backslash P_{0}$, which are rational convex polyhedral sets whose associated cones are the 3-dimensional face $P_{0}$ of $\sigma_{\{2,4\}}$ and $\sigma_{\{2,4\}}$ respectively. Then, for $\underline{n} \in P_{0} \cap \mathbf{Z}^{4}$,

$$
\bar{D}_{\underline{\underline{n}}}-\Delta_{\underline{n}}=\left\{\begin{array}{cl}
0 & \text { if } 2 \mid n_{3}, \\
\frac{1}{2} E_{2}+E_{3}+\frac{1}{2} E_{4} & \text { if } 2 \not n_{3},
\end{array}\right.
$$

and, for $\underline{n} \in P_{1} \cap \mathbf{Z}^{4}$,

$$
\bar{D}_{\underline{n}}-\Delta_{\underline{n}}=\left\{\begin{array}{cl}
0 & \text { if } 2\left|n_{1}, 2\right| n_{3}, \\
\frac{1}{2} E_{4} & \text { if } 2 \not n_{1}, 2 \not n_{3}, \\
\frac{1}{2} E_{2} & \text { if } 2 \not n_{1}, 2 \mid n_{3}, \\
\frac{1}{2} E_{2}+\frac{1}{2} E_{4} & \text { if } 2 \mid n_{1}, 2 \not n_{3} .
\end{array}\right.
$$

This shows that $\bar{D}_{\underline{n}}-\Delta_{\underline{n}}$ is not a periodic function on $\sigma_{\{2,4\}}$ in the sense of Definition 6.2 i.e., in Theorem 4.9 we cannot take $\mathcal{P}$ to be equal to $\Sigma$.

\section{Singularities With SEMi-ABElian $\mathrm{Pic}^{0}$}

The following result is a direct consequence of Lang's conjecture, proven by McQuillan in [21]. Recall that a semi-abelian variety is a commutative algebraic group such that there is an exact sequence

$$
0 \rightarrow \mathbf{G}_{m}^{a} \rightarrow G \rightarrow A \rightarrow 0,
$$

where $A$ is an abelian variety, and $a \in \mathbf{N}$.

Proposition 5.1. Suppose that $G$ is a not necessarily connected commutative algebraic group over an algebraically closed field $\mathbf{k}$ of characteristic 0 , such that $G$ is an extension of a finite abelian group by a semi-abelian variety, $H$ is a finitely generated abelian group, and $\pi: H \rightarrow G(\mathbf{k})$ is a group homomorphism. Suppose that $Y \subset G$ is a closed integral subvariety such that $Y(\mathbf{k}) \cap \pi(H)$ is Zariski dense in $Y$. Then there exist a subgroup $M$ of $H$ and $n_{0} \in H$ such that

$$
\pi(n) \in Y(\mathbf{k}) \text { iff } n \in n_{0}+M \text {. }
$$

Proof. First suppose that $G$ is a semi-abelian variety. Let $\Gamma=\pi(H)$, and

$$
\bar{\Gamma}=\{x \in G(\mathbf{k}) \mid n x \in \Gamma \text { for some } n \in \mathbf{N}\} .
$$

$Y(\mathbf{k}) \cap \bar{\Gamma}$ is Zariski dense in $X$, so by Lang's conjecture (proven in 21]) $Y=b+\Delta$ for some $b \in G(\mathbf{k})$ and some semi-abelian subvariety $\Delta$ of $\Gamma$. By assumption, there exists an $n_{0} \in H$ such that $\pi\left(n_{0}\right) \in b+\Delta$, so we can assume that $b=\pi\left(n_{0}\right)$. Let

$$
M=\{n \in H \mid \pi(n) \in \Delta(k)\},
$$


a subgroup of $H$. We have

$$
n_{0}+M=\{n \in H \mid \pi(n) \in Y(\mathbf{k})\} .
$$

In the case where $G$ is not connected, we have an exact sequence

$$
0 \rightarrow A \rightarrow G \rightarrow F \rightarrow 0,
$$

where $F$ is a finite abelian group and $A$ is a semi-abelian variety. There exists $m_{0} \in$ $H$ such that $y_{0}=\pi\left(m_{0}\right) \in Y(\mathbf{k})$. Thus $Y^{\prime}=Y-y_{0} \subset A$. Suppose that $Z^{\prime} \subset A$ is a closed subvariety containing $\pi(H) \cap Y^{\prime}(\mathbf{k})$. Then $\pi(H) \cap Y(\mathbf{k}) \subseteq Z^{\prime}(\mathbf{k})+y_{0}$. By our assumption, $Y \subset Z^{\prime}+y_{0}$, which implies that $Y^{\prime} \subset Z^{\prime}$. Thus $\pi(H) \cap Y^{\prime}(\mathbf{k})$ is Zariski dense in $Y^{\prime}(\mathbf{k})$.

Let $H^{\prime}=\pi^{-1}(A(\mathbf{k}))$, a subgroup of $H$. By the first part of the proof, there exist a subgroup $M$ of $H^{\prime}$ and $m_{1} \in H^{\prime}$ such that $\pi(n) \in Y^{\prime}(\mathbf{k})$ if and only if $n \in m_{1}+M$. Thus $\pi(n) \in Y(\mathbf{k})$ if and only if $\pi\left(n-m_{0}\right) \in Y^{\prime}(\mathbf{k})$, which holds if and only if $n-m_{0} \in m_{1}+M$, and this is true if and only if $n \in\left(m_{0}+m_{1}\right)+M$.

The conclusion of Proposition [5.1] is false even for $\mathbf{G}_{m}$ ([18]) and for abelian varieties (9], Example 3) over a field of characteristic $p>0$.

Example 5.2. The conclusion of Proposition 5.1 is false for arbitrary commutative algebraic groups over an algebraically closed field $\mathbf{k}$ of characteristic zero.

Proof. We first observe that the only nontrivial integral closed subgroups $G$ of $\mathbf{G}_{a}^{2}$ over $\mathbf{C}$ are the lines through the origin. This can be seen easily. Choose $0 \neq x \in G(\mathbf{C})$. Let $H$ be the line through the origin containing $x$. Then $H$ is a closed subgroup; thus $H \cap G$ is a closed subgroup containing $x$. Since $\sharp\langle x\rangle=\infty$, we have $\sharp(H \cap G)=\infty$. Since both $H$ and $G$ have dimension 1, we have $H=H \cap G=G$. In particular, we see that a translation of an integral nontrivial subgroup of $\mathbf{G}_{a}^{2}$ is defined by the vanishing of a linear equation

$$
a x+b y+c=0 .
$$

If $Y$ is an integral curve and $\sharp\left(Y \cap \mathbf{Z}^{2}\right)=\infty$, then the Zariski closure of $Y \cap \mathbf{Z}^{2}$ in $Y$ is $Y$. Thus, if $Y$ is not a line, it is not the translation of a semi-abelian subvariety of $\mathbf{G}_{a}^{2}$. In particular, as suggested in [29, $Y$ can be taken to be the integral curve in $\mathbf{G}_{a}^{2}$ defined by Pell's equation $y^{2}-2 x^{2}=1$.

Given a proper $\mathbf{k}$-scheme $Z$ over an algebraically closed field $\mathbf{k}$, let $\operatorname{Pic}^{\tau}(Z)$ and $\operatorname{Pic}^{0}(Z)$ be the subsets of $\operatorname{Pic}(Z)$ of invertible sheaves on $Z$ which are numerically equivalent to 0 and algebraically equivalent to 0 respectively. By the theory of the Picard scheme developed in [11] and [24] (cf. [9], section 2) there exists a group scheme $\operatorname{Pic}_{Z}^{\tau}$ such that $\operatorname{Pic}_{Z}^{\tau}(\mathbf{k}) \cong \operatorname{Pic}^{\tau}(Z)$. If $\operatorname{Pic}_{Z}^{0}$ is the connected component of the identity of $\operatorname{Pic}_{Z}^{\tau}$, then $\operatorname{Pic}_{Z}^{0}(\mathbf{k}) \cong \operatorname{Pic}^{0}(Z)$ and $\operatorname{Pic}^{\tau}(Z) / \operatorname{Pic}^{0}(Z)$ is a finite group (Theorem 4 of [20], Proposition 14 of [9]). If $Y$ is another proper $\mathbf{k}$-scheme and $\phi$ : $Z \rightarrow Y$ is a morphism, $\phi$ induces a morphism of group schemes $\mathrm{Pic}_{Y}^{\tau} \rightarrow \mathrm{Pic}_{Z}^{\tau}$, hence also $\operatorname{Pic}_{Y}^{0} \rightarrow \operatorname{Pic}_{Z}^{0}$, such that $\operatorname{Pic}_{Y}^{\tau}(\mathbf{k}) \rightarrow \operatorname{Pic}_{Z}^{\tau}(\mathbf{k})$ is the pullback homomorphism.

Let $f: X \rightarrow \operatorname{spec}(R)$ and $E_{1}, \ldots, E_{r}$ be as in section 4 . Suppose that $\mathbf{k}$ is algebraically closed of characteristic zero. Let

$$
\operatorname{Pic}^{0}(X)=\left\{\mathcal{L} \in \operatorname{Pic}(X) \mid\left(\mathcal{L} \cdot E_{i}\right)=0 \text { for } 1 \leq i \leq r\right\} .
$$

Lemma 5.3. Let $D$ be an effective divisor on $X$ with exceptional support. Then, the induced morphism of algebraic groups $\operatorname{Pic}^{0}(X) \rightarrow \operatorname{Pic}^{0}(D)$ is surjective, and $\operatorname{Pic}^{0}(D)=\operatorname{Pic}^{\tau}(D)$. 
Proof. $\operatorname{Pic}^{0}(D)=\operatorname{Pic}^{\tau}(D)$, since $D$ is a curve. (In our case, it follows from the analysis of [2] that the kernel of the degree map $d: \operatorname{Pic}(D) \rightarrow \mathbf{Z}^{r}$ is connected. Since this kernel is $\operatorname{Pic}^{\tau}(D)$, we have $\operatorname{Pic}^{\tau}(D)=\operatorname{Pic}^{0}(D)$.) $\operatorname{Pic}(X) \rightarrow \operatorname{Pic}(D)$ is surjective by Lemma 14.3 of [19]. Thus if $\mathcal{L} \in \operatorname{Pic}^{0}(D)$, there exists $\mathcal{M} \in \operatorname{Pic}(X)$ such that $\mathcal{M} \otimes \mathcal{O}_{D} \cong \mathcal{L}$. Let $E_{i_{1}}, \ldots, E_{i_{m}}$ be the integral exceptional curves which are not in the support of $D$. Let $r_{i_{j}}=\left(\mathcal{M} \cdot E_{i_{j}}\right)$ for $1 \leq j \leq m$. Since $R$ is Henselian, there exist integral curves $D_{i_{j}}, 1 \leq j \leq m$, which are disjoint from $D$ and $E_{i_{k}}$ if $k \neq j$, and such that $\left(D_{i_{j}} \cdot E_{i_{k}}\right)=1$. Let $\mathcal{N}=\mathcal{M} \otimes \mathcal{O}_{X}\left(-\sum_{j=1}^{m} r_{i_{j}} D_{i_{j}}\right)$. $\mathcal{N} \in \operatorname{Pic}^{0}(X)$ and $\mathcal{N} \otimes \mathcal{O}_{D} \cong \mathcal{L}$.

Lemma 5.4. Suppose that $D_{0}$ is an effective exceptional divisor on $X$. Then, there exists an effective exceptional divisor $D$ on $X$ such that $D_{0} \leq D$ and $\operatorname{Pic}^{0}(X) \rightarrow$ $\operatorname{Pic}^{0}(D)$ is an isomorphism.

Proof. Suppose that $F$ is an effective exceptional divisor with exceptional support such that $-F$ is ample and $D_{0} \leq F$. Then

$$
H^{1}\left(X, \mathcal{O}_{X}^{*}\right) \cong \lim _{\leftarrow} H^{1}\left(X, \mathcal{O}_{n F}^{*}\right) .
$$

There exists $n_{0}>0$ such that $H^{1}\left(X, \mathcal{O}_{F}(-n F)\right)=0$ for $n \geq n_{0}$. The proof of Lemma 1.4 in [2] implies

$$
H^{1}\left(X, \mathcal{O}_{(n+1) F}^{*}\right) \cong H^{1}\left(X, \mathcal{O}_{n F}^{*}\right)
$$

for $n \geq n_{0}$. Thus

$$
H^{1}\left(X, \mathcal{O}_{X}^{*}\right) \cong H^{1}\left(X, \mathcal{O}_{n F}^{*}\right) .
$$

In the proof of the next lemma we will use Chevalley's theorem (cf. Proposition 11 in Chapter III of [26]) which tells us that if $G$ is a commutative algebraic group, then there is an exact sequence of algebraic groups

$$
0 \rightarrow L \rightarrow G \rightarrow B \rightarrow 0,
$$

where $B$ is an abelian variety and $L$ is a (commutative) linear algebraic group. Further, $L \cong \mathbf{G}_{m}^{\beta} \times \mathbf{G}_{a}^{\gamma}$ (cf. [26], Proposition 12 in Chapter III and the corollary to Proposition 8 in Chapter VIII).

Theorem 5.5. Suppose that $\operatorname{Pic}^{0}(X)$ is a semi-abelian variety, and $D$ is an effective divisor with exceptional support. Then $\mathrm{Pic}^{0}(D)$ is semi-abelian.

Proof. The pullback morphism $\Lambda: \operatorname{Pic}^{0}(X) \rightarrow \operatorname{Pic}^{0}(D)$ is the morphism of $\mathbf{k}$-valued points induced by a surjective morphism of algebraic groups (by Lemmas 5.3 and 5.4). By assumption, $\operatorname{Pic}^{0}(X)$ is a semi-abelian variety, so there is an exact sequence of algebraic groups

$$
0 \rightarrow \mathbf{G}_{m}^{\alpha} \rightarrow \operatorname{Pic}^{0}(X) \rightarrow A \rightarrow 0
$$

where $A$ is an abelian variety. There is an exact sequence

$$
0 \rightarrow L \rightarrow \operatorname{Pic}^{0}(D) \rightarrow B \rightarrow 0,
$$

where $B$ is an abelian variety and $L$ is a commutative linear algebraic group. We have $\Lambda\left(\mathbf{G}_{m}^{\alpha}\right) \subset L$, since a rational map of $\mathbf{P}^{1}$ to an abelian variety is trivial (cf. the corollary to Lemma 7 in Chapter III of [26]). Suppose that $\Lambda\left(\mathbf{G}_{m}^{\alpha}\right) \neq L$. We can form the quotient $Z=\mathrm{Pic}^{0}(D) / \Lambda\left(\mathbf{G}_{m}^{\alpha}\right)$, which is as algebraic group (cf. Chapter II of [3]). 
We have a surjective morphism $\bar{\Lambda}: A \rightarrow Z$. There is an extension

$$
0 \rightarrow L_{1} \rightarrow Z \rightarrow C \rightarrow 0,
$$

where $C$ is an abelian variety and $L_{1}$ is a nontrivial commutative linear algebraic group. Then $\bar{\Lambda}^{-1}\left(L_{1}\right)$ is a subgroup of $A$. Since $A$ contains no rational curves, the connected component $B$ of $\bar{\Lambda}^{-1}\left(L_{1}\right)$ containing the identity is an abelian variety which surjects onto $L_{1}$. We have an inclusion

$$
\Gamma\left(L_{1}, \mathcal{O}_{L_{1}}\right) \subset \Gamma\left(B, \mathcal{O}_{B}\right)=\mathbf{k} .
$$

Since $L_{1}$ is affine, $L_{1}=\operatorname{spec}(\mathbf{k})$.

Thus we have a surjection

$$
\Lambda: \mathbf{G}_{m}^{\alpha} \rightarrow L \cong \mathbf{G}_{m}^{\beta} \times \mathbf{G}_{a}^{\gamma}
$$

for some $\beta, \gamma \geq 0$. Suppose that $\gamma>0$. Taking an inclusion and a quotient, we have a surjective homomorphism of algebraic groups, $\phi: \mathbf{G}_{m} \rightarrow \mathbf{G}_{a}$. Every root of unity in $\mathbf{G}_{m}(\mathbf{k}) \cong \mathbf{k}^{*}$ must map to 0 in $\mathbf{G}_{a}(\mathbf{k}) \cong \mathbf{k}$. Thus the kernel $K$ of $\phi$, which is a closed subgroup of $\mathbf{G}_{m}$, is infinite. Thus $K=\mathbf{G}_{m}$, and we have a contradiction, showing that $\gamma=0$. Thus $\operatorname{Pic}^{0}(D)$ is a semi-abelian variety.

Proposition 5.6. Suppose that the reduced exceptional locus $E$ of $X$ is a simple normal crossing divisor. Then $\operatorname{Pic}^{0}(X)$ is a semi-abelian variety if and only if $\operatorname{Pic}^{0}(X) \rightarrow \operatorname{Pic}^{0}(E)$ is an isomorphism.

Proof. Since $E$ is a reduced divisor with normal crossings, it follows from 2], p. 488 , that there is an exact sequence

$$
0 \rightarrow \mathbf{G}_{m}^{N} \rightarrow \operatorname{Pic}^{0}(E) \rightarrow \prod_{i=1}^{r} \operatorname{Pic}^{0}\left(E_{i}\right) \rightarrow 0
$$

for some $N \in \mathbf{N}$. Since each $E_{i}$ is a smooth projective curve, each $\operatorname{Pic}^{0}\left(E_{i}\right)$ is an abelian variety. We thus see that (with our assumptions on $E$ ) $\operatorname{Pic}^{0}(E)$ is a semi-abelian variety, and the sufficiency of the condition follows.

Let $D$ be an effective exceptional divisor such that $\operatorname{Pic}^{0}(X) \cong \operatorname{Pic}^{0}(D)$ and $D \geq E$, as in Lemma 5.3. The analysis of [2] shows that there is a surjection $\operatorname{Pic}^{0}(D) \rightarrow \operatorname{Pic}^{0}(E)$, and the kernel has a composition series with factors isomorphic to $\mathbf{G}_{a}$, and thus is isomorphic to $\mathbf{G}_{a}^{\alpha}$ for some $\alpha$. If $\mathrm{Pic}^{0}(X)$ is a semi-abelian variety, we must then have that the kernel is trivial.

\section{Structure of the Hilbert function $h: \mathbf{N}^{r} \rightarrow \mathbf{N}$}

Let $R$ be a complete normal local ring of dimension two, and let $f: X \rightarrow \operatorname{spec}(R)$ be a resolution of singularities with integral exceptional divisors $E_{1}, \ldots, E_{r}$. Recall that $h(\underline{n})=\ell\left(R / H^{0}\left(X, \mathcal{O}_{X}\left(-D_{\underline{n}}\right)\right)\right)$ for $\underline{n} \in \mathbf{N}^{r}$. In this section we will prove a structure theorem for the Hilbert-Samuel function $h$.

The following result will play an analogous role to Lemma 4.8.

Lemma 6.1. Let $S$ be a subset of $\{1, \ldots, r\}$ and $J=\{1, \ldots, r\} \backslash S$. For any subset $T$ of $J$ and any map $\alpha: T \rightarrow \frac{1}{e_{S}} \mathbf{N}$, there exist a map $\alpha^{h}: J \backslash T \rightarrow \frac{1}{e_{S}} \mathbf{N}$ and an effective divisor $C_{\alpha}$ with support in $\bigcup_{i \in S \cup T} E_{i}$ such that:

(i) $\overline{D_{\underline{n}}}-\Delta_{\underline{n}}=\overline{D_{\underline{m}}}-\Delta_{\underline{m}}$ for $\underline{n}, \underline{m} \in P\left(\alpha, \alpha^{h}\right) \cap \mathbf{Z}^{r}, \underline{n}-\underline{m} \in e_{S} \mathbf{Z}^{r}$. 
(ii) For $\underline{n} \in P\left(\alpha, \alpha^{h}\right) \cap \mathbf{Z}^{r}$, we have

$$
H^{1}\left(X, \mathcal{O}_{X}\left(-\overline{D_{\underline{n}}}\right)\right)=H^{1}\left(X, \mathcal{O}_{C_{\alpha}}\left(-\overline{D_{\underline{n}}}\right)\right) .
$$

In particular, if $S \cup T=\emptyset$ then $H^{1}\left(X, \mathcal{O}_{X}\left(-\overline{D_{\underline{n}}}\right)\right)=0$ for $\underline{n} \in P\left(\alpha, \alpha^{h}\right) \cap$ $\mathbf{Z}^{r}$.

Proof. Let $\alpha^{c}: J \backslash T \rightarrow \frac{1}{e_{S}} \mathbf{N}$ be the map obtained in Lemma 4.8. Then, for $\underline{n} \in P\left(\alpha, \alpha^{c}\right) \cap \mathbf{Z}^{r}$, the $\mathbf{Q}$-divisor $\overline{D_{\underline{n}}}-\Delta_{\underline{n}}$ reaches only a finite number of values $B_{1}, \ldots, B_{t}$. Hence, for every $\underline{n} \in P\left(\bar{\alpha}, \alpha^{c}\right) \cap \mathbf{Z}^{r}$, there exists $k, 1 \leq k \leq t$, such that

$$
\overline{D_{\underline{n}}}=\sum_{j \in J \backslash T} l_{j}^{S}(\underline{n}) \Delta_{j}+\sum_{j \in T} \alpha(j) \Delta_{j}+B_{k} .
$$

Let $F$ be such that $-F$ is ample. From the exact sequence (8) tensored with $\mathcal{O}_{X}\left(-\bar{D}_{\underline{n}}\right)$ (and since $-\bar{D}_{\underline{n}}$ is nef) it follows that there exists $m_{0} \in \mathbf{N}$ such that

$$
H^{1}\left(X, \mathcal{O}_{X}\left(-\bar{D}_{\underline{n}}\right)\right)=H^{1}\left(X, \mathcal{O}_{m_{0} F}\left(-\bar{D}_{\underline{n}}\right)\right) \quad \text { for all } \underline{n} \in P\left(\alpha, \alpha^{c}\right) \cap \mathbf{Z}^{r} .
$$

In an analogous way as in the proof of Lemma 2.7, let $m_{0} F=C_{\alpha}+C_{\alpha}^{\prime}$, where Supp $C_{\alpha} \subseteq \bigcup_{i \in S \cup T} E_{i}$ and Supp $C_{\alpha}^{\prime} \subseteq \bigcup_{j \in J \backslash T} E_{j}$. Then, there exists $b \in \mathbf{N}$ such that, if $\underline{n} \in P\left(\alpha, \alpha^{c}\right) \cap \mathbf{Z}^{r}$ and $l_{j}^{S}(\underline{n}) \geq b$ for $j \in J \backslash T$, then $H^{1}\left(X, \mathcal{O}_{C_{\alpha}^{\prime}}\left(-C_{\alpha}-\bar{D}_{\underline{n}}\right)\right)=$ 0 ; hence

$$
H^{1}\left(X, \mathcal{O}_{m_{0} F}\left(-\overline{D_{\underline{n}}}\right)\right)=H^{1}\left(X, \mathcal{O}_{C_{\alpha}}\left(-\overline{D_{\underline{n}}}\right)\right) .
$$

Therefore, the map $\alpha^{h}: J \backslash T \rightarrow \frac{1}{e_{S}} \mathbf{N}$ given by

$$
\alpha^{h}(j)=\sup \left\{\alpha^{c}(j), b\right\} \quad \text { for } j \in J \backslash T
$$

satisfies the result.

Definition 6.2. A function $\varphi: \mathbf{Z}^{r} \rightarrow \mathbf{Q}$ is called periodic if there exists a subgroup $H$ of $\mathbf{Z}^{r}$ such that $\mathbf{Z}^{r} / H$ is finite and

$$
\varphi(\underline{n})=\varphi(\underline{m}) \quad \text { for } \underline{n}, \underline{m} \in \mathbf{Z}^{r}, \underline{n}-\underline{m} \in H .
$$

For example, from (28) it follows that, for each polyhedral set $P$ in the abstract complex $\mathcal{P}$ in Theorem 4.9, each of the components of the function

$$
P \cap \mathbf{Z}^{r} \rightarrow \mathbf{E}_{\mathbf{Q}} \cong \mathbf{Q}^{r}, \quad \underline{n} \mapsto \overline{D_{\underline{n}}}-\Delta_{\underline{n}}
$$

can be extended to a periodic function on $\mathbf{Z}^{r}$.

Proposition 6.3. There exists an abstract complex of polyhedral sets $\mathcal{P}$ subdividing the fan $\Sigma$ in Corollary 4.3 with the same associated cones, such that, for $\underline{n} \in \mathbf{N}^{r}$,

$$
h(\underline{n})=Q(\underline{n})+L(\underline{n})+\varphi(\underline{n}),
$$

where, for each $P \in \mathcal{P}$, we have:

(i) For $\underline{n} \in P \cap \mathbf{N}^{r}, Q(\underline{n})$ is equal to a polynomial of degree two with coefficients in $\mathbf{Q}$.

(ii) For $\underline{n} \in P \cap \mathbf{N}^{r}$ we have $L(\underline{n})=\sum_{i=1}^{r} \varphi_{i}(\underline{n}) n_{i}$, where $\varphi_{i}$ is equal to a periodic function, for $1 \leq i \leq r$.

(iii) $\varphi$ is bounded.

More precisely, if $P \subseteq \sigma_{S}$ (there always exists such an $S$ ), then

$\left(\mathrm{i}^{\prime}\right)$ the polynomial in (i) has coefficients in $\frac{1}{2\left(e_{S}\right)^{2}} \mathbf{Z}$, and is the same for all $P^{\prime} \in \mathcal{P}$ such that $P^{\prime} \subseteq \sigma_{S}$; and 
(ii') the functions $\varphi_{i}$ in (ii) satisfy

$$
\varphi_{i}(\underline{n})=\varphi_{i}(\underline{m}) \quad \text { for } \underline{n}, \underline{m} \in P \cap \mathbf{Z}^{r}, \underline{n}-\underline{m} \in e_{S} \mathbf{Z}^{r} .
$$

Proof. From the Riemann-Roch formula (6),

$$
\begin{aligned}
h(\underline{n}) & =\ell\left(R / H^{0}\left(X, \mathcal{O}_{X}\left(-\bar{D}_{\underline{n}}\right)\right)\right) \\
& =\frac{1}{2}\left(-\left(K_{X} \cdot \bar{D}_{\underline{n}}\right)-\left(\bar{D}_{\underline{n}}\right)^{2}\right)+h^{1}\left(X, \mathcal{O}_{X}\right)-h^{1}\left(X, \mathcal{O}_{X}\left(-\bar{D}_{\underline{n}}\right)\right) \\
& =\frac{1}{2}\left(-\left(K_{X} \cdot \Delta_{\underline{n}}\right)-\left(\Delta_{\underline{n}}\right)^{2}\right)+\frac{1}{2}\left(-K_{X} \cdot\left(\bar{D}_{\underline{n}}-\Delta_{\underline{n}}\right)-\left(\bar{D}_{\underline{n}}-\Delta_{\underline{n}}\right)^{2}\right) \\
& -\left(\left(\bar{D}_{\underline{n}}-\Delta_{\underline{n}}\right) \cdot \Delta_{\underline{n}}\right)+h^{1}\left(X, \mathcal{O}_{X}\right)-h^{1}\left(X, \mathcal{O}_{X}\left(-\bar{D}_{\underline{n}}\right)\right) .
\end{aligned}
$$

If $\Sigma$ is the fan in Corollary 4.3, and we take $\mathcal{P}$ to be the abstract complex of polyhedral sets in Theorem 4.9, which subdivides $\Sigma$ with the same associated cones, then, from Corollary 4.3 and Theorem 4.9 it follows that

$$
h(\underline{n})=Q(\underline{n})+L(\underline{n})+\varphi_{0}(\underline{n})-h^{1}\left(X, \mathcal{O}_{X}\left(-\overline{D_{\underline{n}}}\right)\right),
$$

where, for each $P \in \mathcal{P}, Q$ (resp. L) satisfies (i) and $\left(\mathrm{i}^{\prime}\right)$ (resp. (ii) and $\left(\mathrm{ii}^{\prime}\right)$ ), and $\varphi_{0}$ also satisfies $\left(\mathrm{ii}^{\prime}\right)$.

The function $\underline{n} \mapsto h^{1}\left(X, \mathcal{O}_{X}\left(-\overline{D_{\underline{n}}}\right)\right)$ is bounded by Lemma 2.4 .

Corollary 6.4. There exists an abstract complex of polyhedral sets $\mathcal{P}$ subdividing the fan $\Sigma$ in Corollary 4.3 with the same associated cones such that, for each $P \in \mathcal{P}$, there exist $m_{P} \in \mathbf{N}$, a set $\left\{Q_{P, i}(\underline{n})\right\}_{i=1}^{m_{P}}$ of polynomials of degree two, and a function $\gamma_{P}: P \cap \mathbf{Z}^{r} \rightarrow\left\{1, \ldots, m_{P}\right\}$ such that

$$
h(\underline{n})=Q_{P, \gamma_{P}(\underline{n})}(\underline{n}) \quad \text { for } \underline{n} \in P \cap \mathbf{Z}^{r} .
$$

Definition 6.5. Suppose that $P \subset \mathbf{Q}^{r}$ is a polyhedral set. We say that a function $\varphi: P \cap \mathbf{Z}^{r} \rightarrow \mathbf{Q}$ is semi-periodic if

(1) $I=$ Image $\varphi$ is a finite set.

(2) For each $i \in I$, there exists a finite set $\Omega_{i}$, and for each $l \in \Omega_{i}$ we associate an element $\underline{m}_{l} \in \mathbf{Z}^{r}$ and a subgroup $\Delta_{l} \subseteq \mathbf{Z}^{r}$ such that for $\underline{n} \in P \cap \mathbf{Z}^{r}$,

$$
\varphi(\underline{n}) \geq i \Leftrightarrow \underline{n} \in \bigcup_{l \in \Omega_{i}}\left(\underline{m}_{l}+\Delta_{l}\right)
$$

Note that periodic implies semi-periodic, and the sum and product of semiperiodic functions are periodic.

Theorem 6.6. Suppose that the residue field $\mathbf{k}$ of $R$ is an algebraically closed field of characteristic 0 and, either $r \leq 2$, or $r>2$ and $\operatorname{Pic}^{0}(X)$ is a semi-abelian variety. Then, there exists an abstract complex of polyhedral sets $\mathcal{P}$ subdividing the fan $\Sigma$ in Corollary 4.3 with the same associated cones, such that, for $\underline{n} \in \mathbf{N}^{r}$,

$$
h(\underline{n})=Q(\underline{n})+L(\underline{n})+\varphi(\underline{n}),
$$

where, for each $P \in \mathcal{P}$, we have:

(i) For $\underline{n} \in P \cap \mathbf{Z}^{r}, Q(\underline{n})$ is equal to a polynomial of degree two with coefficients in $\mathbf{Q}$.

(ii) For $\underline{n} \in P \cap \mathbf{Z}^{r}$ we have $L(\underline{n})=\sum_{i=1}^{r} \varphi_{i}(\underline{n}) n_{i}$, where $\varphi_{i}$ is equal to a periodic function for $1 \leq i \leq r$.

(iii) For $\underline{n} \in P \cap \mathbf{Z}^{r}, \varphi(\underline{n})$ is equal to a semi-periodic function. 
Proof. Let $\Sigma$ be the fan of Corollary 4.3, and $\mathcal{P}$ the abstract complex of polyhedral sets in Theorem 4.9 (and Proposition 6.3) which subdivides $\Sigma$ with the same associated cones.

By Lemmas 6.1 and 4.6, the abstract complex $\mathcal{P}$ can be refined to an abstract complex of polyhedral sets $\mathcal{P}^{\prime}$ subdividing $\Sigma$ with the same associated cones and such that, for every $P \in \mathcal{P}^{\prime}$, there exist disjoint subsets $S, T$ of $\{1, \ldots, r\}$ and a map $\alpha: T \rightarrow \frac{1}{e_{S}} \mathbf{N}$ such that $P \subseteq P_{S}\left(\alpha, \alpha^{h}\right)$, where $\alpha^{h}$ is the map assigned to $\alpha$ in Lemma 6.1

We will prove that for $P \in \mathcal{P}^{\prime}$, the function $\underline{n} \mapsto h^{1}\left(X, \mathcal{O}_{X}\left(-\overline{D_{\underline{n}}}\right)\right)$ is a semiperiodic function on $P \cap \mathbf{Z}^{r}$.

Fix a polyhedral set $P \in \mathcal{P}^{\prime}$. There are $S$ and $\alpha: T \rightarrow \frac{1}{e_{S}} \mathbf{N}$ such that $P \subseteq$ $P\left(\alpha, \alpha^{h}\right)$. If $S \cup T=\emptyset$, then $h^{1}\left(X, \mathcal{O}_{X}\left(-\overline{D_{n}}\right)\right)=0$ for $\underline{n} \in P \cap \mathbf{Z}^{r}$. So, suppose that $S \bigcup T \neq \emptyset$. Then there exists an effective divisor $C_{\alpha}$ with support in $\bigcup_{i \in S \cup T} E_{i}$ such that (i) and (ii) in Lemma 6.1 hold. Let $\left\{\underline{n}_{1}, \ldots, \underline{n}_{t}\right\} \subseteq P \cap \mathbf{Z}^{r}$ be such that $\underline{n}_{i}-\underline{n}_{k} \notin e_{S} \mathbf{Z}^{r}$ for $i \neq k$, and for all $\underline{n} \in P \cap \mathbf{Z}^{r}$ there exists $\underline{n}_{k}$ such that $\underline{n}-\underline{n}_{k} \in e_{S} \mathbf{Z}^{r}$, i.e.

$$
P \cap \mathbf{Z}^{r}=\bigcup_{k=1}^{t} P \cap\left(\underline{n}_{k}+e_{S} \mathbf{Z}^{r}\right)
$$

and the union is disjoint. Let $H$ be the subgroup of $e_{S} \mathbf{Z}^{r}$ given by the intersection with $e_{S} \mathbf{Z}^{r}$ of all hyperplanes $L(\underline{m})=0$, where $L$ is an integral linear form such that there exists $b \in \mathbf{Z}$ with $P \subseteq\left\{\underline{n} \in \mathbf{Q}^{r} \mid L(\underline{n})=b\right\}$. Then

$$
P \cap \mathbf{Z}^{r}=\bigcup_{k=1}^{t} P \cap\left(\underline{n}_{k}+H\right),
$$

and the union is disjoint. Note that $H \subseteq\left\{\underline{m} \in \mathbf{Q} \mid l_{j}^{S}(\underline{m})=0 \quad \forall j \in T\right\}$, since $P \subseteq P\left(\alpha, \alpha^{h}\right)$. Thus, for $\underline{n} \in P \cap\left(\underline{n}_{k}+H\right)$,

$$
\overline{D_{\underline{n}}}=\Delta_{\underline{n}}+\left(\overline{D_{\underline{n}_{k}}}-\Delta_{\underline{n}_{k}}\right)=\Delta_{\underline{n}-\underline{n}_{k}}^{S}+\overline{D_{\underline{n}_{k}}}=\sum_{j \notin S \cup T} l_{j}^{S}\left(\underline{n}-\underline{n}_{k}\right) \Delta_{j}+\overline{D_{\underline{n}_{k}}} .
$$

Therefore, for

$$
\underline{m} \in\left(-\underline{n}_{k}+P\right) \cap H=P \cap\left(\underline{n}_{k}+H\right)-\underline{n}_{k},
$$

$\sum_{j \notin S \cup T} l_{j}^{S}(\underline{m}) \Delta_{j}$ is a divisor. We can, if necessary, replace $H$ with the subgroup spanned by $\bigcup_{k}\left(-\underline{n}_{k}+P\right) \cap H$. Thus, for all $\underline{m} \in H, \sum_{j \notin S \cup T} l_{j}^{S}(\underline{m}) \Delta_{j}$ is a divisor. Then, since Supp $C_{\alpha} \subseteq \bigcup_{i \in S \cup T} E_{i}$, we have a group homomorphism

$$
\pi: H \rightarrow \operatorname{Pic}^{0}\left(C_{\alpha}\right), \quad \underline{m} \mapsto \sum_{j \notin S \cup T} l_{j}^{S}(\underline{m}) \Delta_{j} \cdot C_{\alpha},
$$

such that

$$
\mathcal{O}_{C_{\alpha}}\left(\overline{D_{\underline{n}}}\right)=\pi\left(\underline{n}-\underline{n}_{k}\right)+\mathcal{O}_{C_{\alpha}}\left(\overline{D_{\underline{n}_{k}}}\right) \quad \text { for } \underline{n} \in P \cap\left(\underline{n}_{k}+H\right) .
$$

For fixed $k, 1 \leq k \leq t$, the sets

$$
\Omega_{k, i}=\left\{\mathcal{L} \in \operatorname{Pic}^{0}\left(C_{\alpha}\right) \mid h^{1}\left(C, \mathcal{L}^{-1} \otimes \mathcal{O}_{C_{\alpha}}\left(-\overline{D_{\underline{n}_{k}}}\right)\right) \geq i\right\}, \quad \text { for } i \in \mathbf{N},
$$

are closed sets of $\operatorname{Pic}^{0}\left(C_{\alpha}\right)$ that define a chain

$$
\ldots \Omega_{k, i+1} \subseteq \Omega_{k, i} \subseteq \ldots \subset \Omega_{k, 0}=\operatorname{Pic}^{0}\left(C_{\alpha}\right)
$$


(cf. the proof of Theorem 8 in $[9]$ ). The chain is stationary, so there exists $\omega_{k}$ such that $\Omega_{k, i}=\Omega_{k, \omega_{k}}$ for $i \geq \omega_{k}$.

By (ii) in Lemma 6.1 (31) and (32), we have

$$
h^{1}\left(X, \mathcal{O}_{X}\left(-\overline{D_{\underline{n}}}\right)\right) \geq i \Longleftrightarrow \pi\left(\underline{n}-\underline{n}_{k}\right) \in \Omega_{k, i} \quad \text { for } \underline{n} \in P \cap\left(\underline{n}_{k}+H\right) .
$$

Suppose first that $r \leq 2$. Since $S \cup T \neq \emptyset$, then $\sharp(\{1, \ldots, r\} \backslash(S \cup T)) \leq 1$, and the semi-periodicity follows as in Theorem 2.8 ([9], Theorem 8).

Now suppose that $r>2$; thus $\operatorname{Pic}^{0}(X)$ is a semi-abelian variety. By Theorem 5.5. $\operatorname{Pic}^{0}\left(C_{\alpha}\right)$ is also a semi-abelian variety. For fixed $k, 1 \leq k \leq t$, let us consider the group homomorphism $\pi: H \rightarrow \operatorname{Pic}^{0}\left(C_{\alpha}\right)$ in (30) and, for $1 \leq i \leq l_{k}$, the closed set $\Omega_{k, i}$ of $\operatorname{Pic}^{0}\left(C_{\alpha}\right)$ in (32). From Proposition 5.1 applied to $\pi$ and each of the irreducible components of the Zariski closure in $\operatorname{Pic}^{0}\left(C_{\alpha}\right)$ of $\Omega_{k, i} \cap \pi(H)$, we conclude that there exist a finite set $\Delta_{k, i}$ and, for each $\delta \in \Delta_{k, i}, \underline{m}_{\delta} \in H$ and a subgroup $M_{\delta}$ of $H$ such that

$$
\pi(\underline{m}) \in \Omega_{k, i} \Longleftrightarrow \underline{m} \in \bigcup_{\delta \in \Delta_{k, i}}\left(\underline{m}_{\delta}+M_{\delta}\right) .
$$

Let $\psi_{k, i}: H \rightarrow \mathbf{N}$ be defined by

$$
\psi_{k, i}(\underline{m})=\left\{\begin{array}{cc}
1 & \text { if } \underline{m} \in \bigcup_{\delta \in \Delta_{k, i}}\left(\underline{m}_{\delta}+M_{\delta}\right), \\
0 & \text { otherwise. }
\end{array}\right.
$$

Let $\psi_{k}=\sum_{i=1}^{\omega_{k}} \psi_{k, i}$, and

$$
\psi: \bigcup_{k=1}^{t}\left(\underline{n}_{k}+H\right) \rightarrow \mathbf{N}, \quad \psi(\underline{n})=\psi_{k}\left(\underline{n}-\underline{n}_{k}\right) \quad \text { if } \underline{n} \in\left(\underline{n}_{k}+H\right),
$$

which is well defined since the union in (29) is disjoint. By (33) we have

$$
h^{1}\left(X, \mathcal{O}_{X}\left(-\overline{D_{\underline{n}}}\right)\right)=\psi(\underline{n}) \quad \text { for } \underline{n} \in P \cap \mathbf{Z}^{r} .
$$

Hence the result follows.

\section{Rationality of the SERIES DEFined By $h$}

In this section we will derive from Theorem [6.6] the rationality of the series $\sum_{\underline{n} \in \mathbf{N}^{r}} h(\underline{n}) \underline{t} \underline{n}$ when the conditions of Theorem 6.6 hold (Theorem 7.7).

A cone $\sigma$ is strongly convex if $\{0\}$ is the maximal linear subspace contained in $\sigma$. A polyhedral set $P$ in $\mathbf{Q}^{r}$ is a module over its associated cone $\sigma_{P}$ in the sense that $\sigma_{P}+P \subset P$.

Theorem 7.1. Let $P$ be a polyhedral set in $\mathbf{Q}^{r}$ whose associated cone $\sigma_{P}$ is strongly convex. Then $P \cap \mathbf{Z}^{r}$ is a finitely generated module over the semigroup $\sigma_{P} \cap \mathbf{Z}^{r}$.

Let $P=\left\{\underline{n} \in \mathbf{Q}^{r} \mid L_{i}(\underline{n}) \geq b_{i}, 1 \leq i \leq m\right\}$, where $m \in \mathbf{N}$ and, for $1 \leq i \leq m$, $L_{i}$ is an integral linear form on $\mathbf{Q}^{r}$ and $\underline{b}=\left(b_{1}, \ldots, b_{m}\right) \in \mathbf{Z}^{r}$, as in (21). Define $L: \mathbf{Z}^{r} \rightarrow \mathbf{Z}^{m}$ by $\underline{v} \rightarrow\left(L_{1}(\underline{v}), \ldots, L_{m}(\underline{v})\right) . \quad L$ is $1-1$, since $\sigma_{P}$ contains only the trivial linear subspace. Let $D_{+}, D_{-} \subset \mathbf{Q}^{m}$ be the regions

$$
\begin{aligned}
& D_{+}=\left\{\left(x_{1}, \ldots, x_{m}\right) \mid x_{i} \geq 0,1 \leq i \leq m\right\}, \\
& D_{-}=\left\{\left(x_{1}, \ldots, x_{m}\right) \mid x_{i} \leq 0,1 \leq i \leq m\right\} .
\end{aligned}
$$


Set $\bar{P}=P \cap \mathbf{Z}^{r}, \overline{\sigma_{P}}=\sigma_{P} \cap \mathbf{Z}^{r} . L$ gives 1-1 correspondences between $\overline{\sigma_{P}}$ and Image $(L) \cap D_{+}$and between $\bar{P}$ and Image $(L) \cap\left(\underline{b}+D_{+}\right)$. There is a partial order on $\mathbf{Q}^{m}, \underline{v}_{1} \leq \underline{v}_{2}$ if all components of $\underline{v}_{1}$ are $\leq$ the corresponding components of $\underline{v}_{2}$.

Definition 7.2. We say that $\underline{v} \in \bar{P}$ is a minimal generator if $\underline{v}=\underline{w}+\underline{v}_{0}$ with $\underline{w} \in \bar{P}, \underline{v}_{0} \in \overline{\sigma_{P}}$ implies $\underline{v}=\underline{w}$.

Theorem 7.1 is an immediate consequence of the following lemma:

Lemma 7.3. (i) The set of minimal generators of $\bar{P}$ generates $\bar{P}$ as a $\overline{\sigma_{P}}$-module.

(ii) If $\underline{v}$ is a minimal generator of $\bar{P}$, then

$$
\left(L(\underline{v})+D_{-}\right) \cap L(\bar{P})=\{L(\underline{v})\} .
$$

(iii) There are only finitely many minimal generators.

Proof. We first prove (i). Suppose that $\underline{w} \in \bar{P}$. If $\underline{w}$ is a minimal generator, we are done. Else, there exists $\underline{w}_{1} \in \bar{P}$ and $\underline{v}_{1} \in \overline{\sigma_{P}}$ such that $\underline{v}_{1} \neq 0$ and $\underline{w}=\underline{w}_{1}+\underline{v}_{1}$.

If $\underline{w}_{1}$ is not a minimal generator, we can repeat. In this way either we realize $\underline{w}$ as a sum $\underline{w}=\underline{w}^{\prime}+\underline{v}^{\prime}$ with $\underline{w}^{\prime}$ a minimal generator, $\underline{v}^{\prime} \in \overline{\sigma_{P}}$, or we construct an infinite sequence

$$
\underline{w}=\underline{w}_{1}+\underline{v}_{1}, \underline{w}_{1}=\underline{w}_{2}+\underline{v}_{2}, \cdots, \underline{w}_{i}=\underline{w}_{i+1}+\underline{v}_{i+1}, \cdots
$$

with $\underline{0} \neq \underline{v}_{i} \in \overline{\sigma_{P}}$ and $\underline{w}_{i} \in \bar{P}$ for all $i$. We have then an infinite sequence of integral vectors

$$
L(\underline{w})>L\left(\underline{w}_{1}\right)>\cdots>L\left(\underline{w}_{i}\right)>\cdots
$$

in the region $\underline{b}+D_{+}$, a contradiction.

Now we prove (ii). Suppose that $\underline{v}$ is a minimal generator which does not satisfy this property. Set $\underline{w}=L(\underline{v})$. Then there exists $\underline{w} \neq \underline{w}_{1} \in L(\bar{P}) \cap\left(\underline{w}+D_{-}\right)$. There is $\underline{v}_{1} \in \bar{P}$ such that $\underline{w}_{1}=L\left(\underline{v}_{1}\right)$. Also,

$$
L\left(\underline{v}-\underline{v}_{1}\right)=\underline{w}-\underline{w}_{1} \in D_{+} \cap \operatorname{Image}(L)
$$

implies $\underline{v}-\underline{v}_{1} \in \overline{\sigma_{P}}$, and $\underline{v}=\underline{v}_{1}+\left(\underline{v}-\underline{v}_{1}\right)$ implies $\underline{v}$ is not a minimal generator, a contradiction.

Finally, we prove (iii). Let $\left\{\underline{v}_{i}\right\}_{i \in I}$ be the set of minimal generators of $\bar{P}$ over $\overline{\sigma_{P}}$. To each minimal generator $\underline{v}_{i}$, associate

$$
\underline{u}_{i}=L\left(\underline{v}_{i}\right)-\underline{b} \in D_{+} \cap \mathbf{Z}^{m}=\mathbf{N}^{m} .
$$

The $\underline{u}_{i}$ are pairwise not comparable by our partial order by (ii). Let $J$ be the ideal

$$
J=\left(\underline{x}^{\underline{u}} \mid i \in I\right) \subset k\left[x_{1}, \ldots, x_{m}\right] .
$$

$J$ is finitely generated and $\left\{\underline{x}^{\underline{u}} \mid i \in I\right\}$ is a minimal set of generators of $J$, so $I$ is finite.

Corollary 7.4. Let $P$ be a polyhedral set in $\mathbf{Q}^{r}$ whose associated cone $\sigma_{P}$ is strongly convex. Then, there exist a polynomial $p\left(t_{1}, \ldots, t_{r}\right) \in \mathbf{Z}\left[t_{1}, \ldots, t_{r}\right], s \in \mathbf{N}$, and nonzero $\underline{a}_{1}, \ldots, \underline{a}_{s} \in \mathbf{N}^{r}$ such that

$$
\sum_{\underline{n} \in P \cap \mathbf{Z}^{r}} \underline{t}^{\underline{n}}=\underline{t}^{\underline{b}} \frac{p\left(t_{1}, \ldots, t_{r}\right)}{\prod_{i=1}^{s}\left(1-\underline{t}^{\underline{a}} \underline{a}^{i}\right)} .
$$


Proof. If $\mathbf{k}$ is a field, then

$$
\mathbf{k}\left[\sigma_{P} \cap \mathbf{Z}^{r}\right]=\bigoplus_{\underline{n} \in \sigma_{P} \cap \mathbf{Z}^{r}} \mathbf{k} \underline{t}^{\underline{n}}
$$

is a $\mathbf{N}^{r}$-graded $\mathbf{k}$-algebra, which is finitely generated over $\mathbf{k}$ (28, Theorem I.3.1). By Theorem 7.1 ,

$$
\mathbf{k}\left[P \cap \mathbf{Z}^{r}\right]=\bigoplus_{\underline{n} \in P \cap \mathbf{Z}^{r}} \mathbf{k} \underline{t}^{\underline{n}}
$$

is a finitely generated $\mathbf{Z}^{r}$-graded $k\left[\sigma_{P} \cap \mathbf{Z}^{r}\right]$-module. Its Hilbert series is

$$
\sum_{\underline{\alpha} \in \mathbf{Z}^{r}} \operatorname{dim} \mathbf{k}\left[P \cap \mathbf{Z}^{r}\right]_{\underline{\alpha}} \underline{\underline{\underline{\alpha}}} \underline{\underline{\alpha}}
$$

and

$$
\operatorname{dim} \mathbf{k}\left[P \cap \mathbf{Z}^{r}\right]_{\underline{\alpha}}= \begin{cases}1 & \text { if } \underline{\alpha} \in P \cap \mathbf{Z}^{r}, \\ 0 & \text { otherwise. }\end{cases}
$$

By [28], Theorem I.2.3, we conclude the result.

Theorem 7.5. Let $P \subseteq \mathbf{Q}_{\geq 0}^{r}$ be a polyhedral set in $\mathbf{Q}^{r}$. Let $M \subseteq \mathbf{Z}^{r}$ be a subgroup and $\underline{m} \in \mathbf{Z}^{r}$. Then, there exist a polynomial $p\left(t_{1}, \ldots, t_{r}\right) \in \mathbf{Z}\left[t_{1}, \ldots, t_{r}\right], s \in \mathbf{N}$, and nonzero $\underline{a}_{1}, \ldots, \underline{a}_{s} \in \mathbf{N}^{r}$ such that

$$
\sum_{\underline{n} \in P \cap(\underline{m}+M)} \underline{t}^{\underline{n}}=\frac{p\left(t_{1}, \ldots, t_{r}\right)}{\prod_{i=1}^{s}\left(1-\underline{t}^{\underline{a}}\right)} .
$$

Proof. We have

$$
P \cap(\underline{m}+M)=\underline{m}+((-\underline{m}+P) \cap M)
$$

if the intersection is nonempty. The set $-\underline{m}+P$ is a polyhedral set in $\mathbf{Q}^{r}$. Write

$$
M=\bigoplus_{j=1}^{r^{\prime}} \mathbf{Z} \underline{m}_{j}
$$

for some $r^{\prime} \leq r$. Set

$$
M_{\mathbf{Q}}=M \otimes \mathbf{z} \mathbf{Q}=\bigoplus_{j=1}^{r^{\prime}} \mathbf{Q} \underline{m}_{j} \subseteq \mathbf{Q}^{r}
$$

and $P^{\prime}=(-\underline{m}+P) \cap M_{\mathbf{Q}}$. With respect to the basis $\left\{\underline{m}_{1}, \ldots, \underline{m}_{r^{\prime}}\right\}$, we can identify $M$ with $\mathbf{Z}^{r^{\prime}}$ and $M_{\mathbf{Q}}$ with $\mathbf{Q}^{r^{\prime}}$. Then, $P^{\prime}$ is a polyhedral set in $\mathbf{Q}^{r^{\prime}}$ whose associated cone is strongly convex. Corollary 7.4 implies

$$
\sum_{\left(\lambda_{1}, \ldots, \lambda_{r^{\prime}}\right) \in P^{\prime} \cap \mathbf{Z}^{r^{\prime}}} T_{1}^{\lambda_{1}} \ldots T_{r^{\prime}}^{\lambda_{r^{\prime}}}=\underline{T}-\underline{\beta} \frac{p^{\prime}\left(T_{1}, \ldots, T_{r}\right)}{\prod_{i=1}^{s}\left(1-\underline{T}^{\underline{\alpha}_{i}}\right)}
$$

for some $p^{\prime}\left(T_{1}, \ldots, T_{r}\right) \in \mathbf{Z}\left[T_{1}, \ldots, T_{r^{\prime}}\right]$, where the $\underline{\alpha}_{i}=\left(\alpha_{i, 1}, \ldots, \alpha_{i, r^{\prime}}\right) \in \mathbf{Z}^{r^{\prime}}$, $1 \leq i \leq s$, are such that $\left\{\sum_{j=1}^{r^{\prime}} \alpha_{i, j} \underline{m}_{j}\right\}_{i=1}^{s}$ generate $\sigma_{P} \cap M$ as k-algebra. Since

$$
\sum_{\underline{n} \in P \cap(\underline{m}+M)} \underline{t}^{\underline{n}}=\underline{t} \underline{\underline{m}} \sum_{\left(\lambda_{1}, \ldots \lambda_{r^{\prime}}\right) \in P^{\prime} \cap \mathbf{Z}^{r^{\prime}}}\left(\underline{t}^{\underline{m}_{1}}\right)^{\lambda_{1}} \ldots\left(\underline{t}^{\underline{m}}{\underline{r^{\prime}}}^{\lambda^{\lambda_{r^{\prime}}}}\right.
$$


we conclude that

$$
\sum_{\underline{n} \in P \cap(\underline{m}+M)} \underline{t}^{\underline{n}}=\frac{\underline{t} \underline{\underline{c}} p\left(t_{1}, \ldots, t_{r}\right)}{\underline{t}^{\underline{d}} \prod_{i=1}^{s}\left(\underline{t}^{\underline{a_{i}}}-\underline{t}^{\underline{b}}\right)},
$$

where $\underline{\underline{t}} \underline{\underline{c}} p\left(t_{1}, \ldots, t_{r}\right)$ and $\underline{t} \underline{\underline{d}} \prod\left(\underline{t}^{\underline{\underline{a}}}{ }_{i}-\underline{t}^{\underline{b}}\right)$ are relatively prime in $\mathbf{Q}\left[t_{1}, \ldots, t_{r}\right]$, and $\underline{b}_{i}-\underline{a}_{i}=\sum_{j=1}^{r^{\prime}} \alpha_{i, j} \underline{m}_{j}$. Since $P \subseteq \mathbf{Q}_{\geq 0}^{r}$,

$$
\sum_{\underline{n} \in P \cap(\underline{m}+M)} \underline{t^{\underline{n}}} \in \mathbf{Q}\left[\left[t_{1}, \ldots, t_{r}\right]\right] \cap \mathbf{Q}\left(t_{1}, \ldots, t_{r}\right)=\mathbf{Q}\left[t_{1}, \ldots, t_{r}\right]_{\left(t_{1}, \ldots, t_{r}\right)} .
$$

Thus $\underline{d}=0$, and $\underline{a}_{i}=0$ or $\underline{b}_{i}=0$ for all $i$.

By formal differentiation of both sides of [7.5] we obtain the following corollary.

Corollary 7.6. Let $P \subseteq \mathbf{Q}_{>0}^{r}$ be a polyhedral set in $\mathbf{Q}^{r}$. Let $\underline{m} \in \mathbf{Z}^{r}$, let $M<\mathbf{Z}^{r}$ be a subgroup, and suppose that $q(\underline{n})$ is a polynomial in $\mathbf{Q}\left[t_{1}, \ldots, t_{r}\right]$. Then, there exist $s \in \mathbf{N}$, nonzero $\underline{a}_{1}, \ldots, \underline{a}_{s} \in \mathbf{N}^{r}, d_{i} \in \mathbf{N}$ and a polynomial $p\left(t_{1}, \ldots, t_{r}\right) \in$ $\mathbf{Q}\left[t_{1}, \ldots, t_{r}\right]$ such that

$$
\sum_{\underline{n} \in P \cap(\underline{m}+M)} q(\underline{n}) \underline{t}^{\underline{n}}=\frac{p\left(t_{1}, \ldots, t_{r}\right)}{\prod_{i=1}^{s}\left(1-\underline{t}^{\underline{a}}\right)^{d_{i}}}
$$

with $d_{i} \leq \operatorname{deg}(q)+1$.

Theorem 7.7. Suppose that $\mathbf{k}=R / m$ is an algebraically closed field of characteristic 0 , and either $r \leq 2$, or $r>2$ and $\mathrm{Pic}^{0}(X)$ is a semi-abelian variety. Then, there exist $s \in \mathbf{N}$, nonzero $\underline{a}_{1}, \ldots, \underline{a}_{s} \in \mathbf{N}^{r}, d_{i} \in \mathbf{N}$ and a polynomial $p\left(t_{1}, \ldots, t_{r}\right) \in \mathbf{Q}\left[t_{1}, \ldots, t_{r}\right]$ such that

$$
\sum_{\underline{n} \in \mathbf{N}^{r}} h(\underline{n}) \underline{t} \underline{\underline{n}}=\frac{p\left(t_{1}, \ldots, t_{r}\right)}{\prod_{i=1}^{s}\left(1-\underline{t}^{\underline{\underline{a}}}\right)^{d_{i}}}
$$

In particular, the series

$$
\sum_{\underline{n} \in \mathbf{N}^{r}} h(\underline{n}) \underline{t^{n}}
$$

is a rational series.

Proof. Let notation be as in Theorem 6.6] and its proof. Let $\Sigma$ be the fan consisting of the cones $\sigma_{S}$ of Definition 4.1 and their faces, and let $\mathcal{P}$ be the abstract complex of polyhedral sets subdividing $\Sigma$ of Theorem [6.6. Put a well ordering on $\mathcal{P}$. Let $\alpha=\#(\mathcal{P})$. Then

$$
\sum_{\underline{n} \in \mathbf{N}^{r}} h(\underline{n}) t^{\underline{n}}=\sum_{i=1}^{\alpha}(-1)^{i-1}\left(\sum_{\gamma_{1}<\cdots<\gamma_{i}}\left(\sum_{\underline{n} \in P_{\gamma_{1}} \cap \cdots \cap P_{\gamma_{i}} \cap \mathbf{Z}^{r}} h(\underline{n}) t^{\underline{n}}\right)\right) .
$$

We are reduced to showing that if $a>0$ and $\gamma_{1}<\cdots<\gamma_{a}$, then

$$
\sum_{\underline{n} \in P_{\gamma_{1}} \cap \cdots \cap P_{\gamma_{a}} \cap \mathbf{Z}^{r}} h(\underline{n}) t^{\underline{n}}
$$

is rational. Let $\Lambda=P_{\gamma_{1}} \cap \cdots \cap P_{\gamma_{a}}$, which is a polyhedral set. Let $P=P_{\gamma_{1}}$. By assumption there exists $\sigma_{S} \in \Sigma$ such that $P \subset \sigma_{S}$. With the notation of the proof 
of Theorem [6.6] we have $\left\{\underline{n}_{1}, \ldots, \underline{n}_{\bar{t}}\right\}$ such that $P \cap \mathbf{Z}^{r}$ is the disjoint union

$$
P \cap \mathbf{Z}^{r}=\bigcup_{k=1}^{\bar{t}} P \cap\left(\underline{n}_{k}+e_{S} \mathbf{Z}^{r}\right) \text {. }
$$

Thus

$$
\sum_{\underline{n} \in \Lambda \cap \mathbf{Z}^{r}} h(\underline{n}) t^{\underline{n}}=\sum_{k=1}^{\bar{t}} \sum_{\underline{n} \in \Lambda \cap\left\{\underline{n}_{k}+e_{S} \mathbf{Z}^{r}\right\}} h(\underline{n}) t^{\underline{n}} .
$$

We can now fix $k$ with $1 \leq k \leq \bar{t}$. For $\underline{n} \in \Lambda \cap\left\{\underline{n}_{k}+e_{S} \mathbf{Z}^{r}\right\}$, we have

$$
h(\underline{n})=q(\underline{n})+\psi(\underline{n}),
$$

where $q(\underline{n})$ is a quadratic polynomial and $\psi(\underline{n})=h^{1}\left(X, \mathcal{O}_{X}\left(-\bar{D}_{\underline{n}}\right)\right)$ is a semiperiodic function. We have

$$
\sum_{\underline{n} \in \Lambda \cap\left\{\underline{n}_{k}+e_{S} \mathbf{Z}^{r}\right\}} h(\underline{n}) t^{\underline{n}}=\sum_{\underline{n} \in \Lambda \cap\left\{\underline{n}_{k}+e_{S} \mathbf{Z}^{r}\right\}} q(\underline{n}) t^{\underline{n}}+\sum_{\underline{n} \in \Lambda \cap\left\{\underline{n}_{k}+e_{S} \mathbf{Z}^{r}\right\}} \psi(\underline{n}) t^{\underline{n}} .
$$

By corollary 7.6, the first series is rational.

Let $\Delta_{k, i}$ be the sets of the proof of Theorem 6.6, with associated $\underline{m}_{\delta} \in \mathbf{Z}^{r}$ and subgroup $M_{\delta}$ of $\mathbf{Z}^{r}$ for $\delta \in \Delta_{k, i}$. Let $\omega=\max \psi(\underline{n})$. Let $B_{k}=\Lambda \cap\left\{\underline{n}_{k}+e_{S} \mathbf{Z}^{r}\right\}$. For $\underline{n} \in B_{k}$ we have

$$
\psi(\underline{n}) \geq i \Leftrightarrow \underline{n} \in \bigcup_{\delta \in \Delta_{k, i}}\left(\underline{m}_{\delta}+M_{\delta}\right)
$$

and

$$
\sum_{\underline{n} \in B_{k}} \psi(\underline{n}) t^{\underline{n}}=\sum_{i=1}^{\omega}\left(\sum_{\underline{n} \in B_{k}} \chi_{i}(\underline{n}) t^{\underline{n}}\right)
$$

where

$$
\chi_{i}(\underline{n})= \begin{cases}1 & \text { if } \underline{n} \in \bigcup_{\delta \in \Delta_{k, i}}\left(\underline{m}_{\delta}+M_{\delta}\right), \\ 0 & \text { if } \left.\underline{n} \notin \bigcup_{\delta \in \Delta_{k, i}} \underline{\underline{m}}_{\delta}+M_{\delta}\right) .\end{cases}
$$

Fix $i$ and well order the set $\Delta_{k, i}$. Then

$$
\sum_{\underline{n} \in B_{k}} \chi_{i}(\underline{n}) t^{\underline{n}}=\sum_{j \geq 1}(-1)^{j-1}\left(\sum_{\delta_{1}<\cdots<\delta_{j}}\left(\sum_{\underline{n} \in B_{k} \cap\left(\underline{n}_{\delta_{1}}+M_{\delta_{1}}\right) \cap \cdots \cap\left(\underline{n}_{\delta_{j}}+M_{\delta_{j}}\right)} t^{\underline{n}}\right)\right),
$$

where $\delta_{1}, \ldots, \delta_{j}$ are in $\Delta_{k, i}$. If $\underline{n}_{0} \in\left(\underline{n}_{\delta_{1}}+M_{\delta_{1}}\right) \cap \cdots \cap\left(\underline{n}_{\delta_{j}}+M_{\delta_{j}}\right)$, then

$$
\left(\underline{n}_{\delta_{1}}+M_{\delta_{1}}\right) \cap \cdots \cap\left(\underline{n}_{\delta_{j}}+M_{\delta_{j}}\right)=\underline{n}_{0}+M_{\delta_{1}} \cap \cdots \cap M_{\delta_{j}} .
$$

Thus rationality now follows from Theorem 7.5

Corollary 7.8. Suppose that the divisor class group $\mathrm{Cl}(R)$ of $R$ is an extension of a finite group by a semi-abelian variety, and $f: X \rightarrow \operatorname{spec}(R)$ is a resolution of singularites. Then the Poincaré series

$$
\sum_{\underline{n} \in \mathbf{N}^{r}} h(\underline{n}) \underline{\underline{n}}
$$

is a rational series. 
Proof. $\mathrm{Cl}(R)$ is an extension of a finite group by $\operatorname{Pic}^{0}(X)$ [19]. The result is then immediate from Theorem 7.7

\section{IRrationality in CHARACTERISTIC $p>0$}

In this section we construct an example where the residue field has positive characteristic $p>0$, there are two irreducible exceptional components, and the associated series are not rational. Recall that if the residue field has characteristic zero, and there are two exceptional components, then the associated series must be rational (Theorem [7.7).

Theorem 8.1. There exists a two-dimensional complete normal local ring $R$ containing a field of characteristic $p>0$, and a resolution of singularities $g: W \rightarrow$ $\operatorname{spec}(R)$ with two exceptional divisors $C_{0}$ and $D$ such that the series

$$
\sum_{m, n \in \mathbf{N}} h^{1}\left(W, \mathcal{O}_{W}\left(-m C_{0}-n D\right)\right) s^{m} t^{n}
$$

and

$$
\sum_{m, n \in \mathbf{N}} \ell\left(R / \Gamma\left(W, \mathcal{O}_{W}\left(-m C_{0}-n D\right)\right)\right) s^{m} t^{n}
$$

are not rational series.

Throughout this section we will use the notation of Example 5 from 9]. In this example, a two-dimensional complete normal local ring $R$ is constructed which contains a field of characteristic $p>0$, and a resolution of singularities $g: W \rightarrow$ $\operatorname{spec}(R)$ with two exceptional divisors $C_{0}$ and $D$. Example 5 from [9] gives an explicit calculation of $h^{1}\left(W, \mathcal{O}_{W}\left(-n C_{0}-n D\right)\right)$ as a function of $n$. This function is bounded, but is not eventually periodic.

We will analyze this example to give counterexamples to the rationality questions which we consider in this paper, in positive characteristic.

Proposition 8.2. Suppose that $0<b \leq a \leq 3 b-3$. Then

$$
h^{1}\left(W, \mathcal{O}_{W}\left(-a C_{0}-b D\right)\right)= \begin{cases}0 & \text { if } a-b \geq 2, \\ 0 & \text { if } a-b=1, a \text { is not a power of } p, \\ 1 & \text { if } a-b=1, a \text { is a power of } p \\ 1 & \text { if } a=b, a+1 \text { is not a power of } p \\ 2 & \text { if } a=b, a+1 \text { is a power of } p .\end{cases}
$$

Proof. The case $a=b$ is proven in Example 5 of [9]. The same arguments extend to prove the theorem. In fact, in the case $a>b$, we also reduce to

$$
H^{1}\left(W, \mathcal{O}_{W}\left(-a C_{0}-b D\right)\right) \cong H^{1}\left(W, \mathcal{O}_{2 C_{0}}\left(-a C_{0}-b D\right)\right) .
$$

In

$$
\begin{aligned}
0 & \rightarrow \mathcal{O}_{C}((a+1)(\eta-P)+(a+1-b) P) \rightarrow \mathcal{O}_{2 C_{0}}\left(-a C_{0}-b D\right) \\
& \rightarrow \mathcal{O}_{C}(a(\eta-P)+(a-b) P) \rightarrow 0
\end{aligned}
$$


$a \geq b+2$ implies all $H^{1}$ in (36) vanish. If $a=b+1$, then

$$
\begin{aligned}
& h^{1}\left(C, \mathcal{O}_{C}((a+1)(\eta-P)+(a+1-b) P)\right)=0, \\
& h^{1}\left(C, \mathcal{O}_{C}(a(\eta-P)+(a-b) P)\right)=h^{1}\left(C, \mathcal{O}_{C}(a(\eta-P)+P)\right. \\
& \quad= \begin{cases}0 & \text { if } a \text { is not a power of } p, \\
1 & \text { if } a \text { is a power of } p .\end{cases}
\end{aligned}
$$

Let $g: W \rightarrow \operatorname{spec}(R)$ be the morphism of Example $5[9]$. Set

$$
\begin{gathered}
a_{i j}=h^{1}\left(W, \mathcal{O}_{W}\left(-i C_{0}-j D\right)\right), \\
h=\sum_{i, j \geq 0} a_{i j} s^{i} t^{t} .
\end{gathered}
$$

Suppose that $h$ is rational, so that there exists a nonzero polynomial

$$
Q=\sum_{i, j=0}^{r} b_{i j} s^{i} t^{j}
$$

such that $Q h$ is a polynomial,

$$
Q h=\sum_{m, n}\left(\sum_{i, j=0}^{r} b_{i j} a_{m-i, n-j}\right) s^{m} t^{n} .
$$

There exists $n_{0}$ such that

$$
\sum_{i, j=0}^{r} b_{i j} a_{m-i, n-j}=0
$$

for $m, n \geq n_{0}$.

Set $m=n+r+1$, and suppose that $n \geq \max \left\{n_{0}+r, 2 r+3,2 p r\right\}$. Then

$$
\sum_{i, j=0}^{r} b_{i j} a_{n+r+1-i, n-j}=0
$$

We have $0<n-j \leq n+r+1-i \leq 3(n-j)-3$ for $0 \leq i, j \leq r$, so by Proposition 8.2, all the $a_{n+r+1-i, n-j}$ vanish in this range, except for

$$
a_{n+1, n}= \begin{cases}0 & \text { if } n+1 \text { is not a power of } p, \\ 1 & \text { if } n+1 \text { is a power of } p .\end{cases}
$$

If we take $n=p^{t}-1$ with $t$ sufficiently large, we then get

$$
0=b_{r 0} a_{n+1, n}=b_{r 0} .
$$

Now assume that $\alpha$ is a natural number such that $0 \leq \alpha \leq r-1$ and $b_{i j}=0$ if $i-j \geq \alpha+1$. We will prove that $b_{i j}=0$ if $i-j \geq \alpha$.

Suppose that $n \geq \max \left\{n_{0}+r, 2 r+3,2 p r\right\}$.

Suppose that $0 \leq i, j \leq r$ and $i-j<\alpha$. Set $a=n+\alpha+1-i, b=n-j$. We then have

$$
\begin{gathered}
a-b=n+\alpha+1-i-(n-j)=\alpha+1+j-i \geq 2, \\
3 b-3=3(n-j)-3 \geq 3 n-3 r-3 \geq n+r \geq n+\alpha+1-i=a .
\end{gathered}
$$


By Proposition 8.2 $a_{n+\alpha+1-i, n-j}=0$ if $i-j<\alpha$. So

$$
\begin{aligned}
0 & =\sum_{i, j=0}^{r} b_{i j} a_{n+\alpha+1-i, n-j} \\
& =\sum_{i-j=\alpha} b_{i j} a_{n+\alpha+1-i, n-j} \\
& = \begin{cases}b_{\alpha, 0} & \text { if } n=p^{t}-1, \\
b_{\alpha+1,1} & \text { if } n=p^{t}, \\
& \vdots \\
b_{r, r-\alpha} & \text { if } n=p^{t}+r-\alpha-1 .\end{cases}
\end{aligned}
$$

(This is where we use $n \geq 2 p r$.) Thus $b_{i j}=0$ if $i-j \geq \alpha$.

We have proved that $b_{i j}=0$ if $i-j \geq r$, so by descending induction we conclude that $b_{i j}=0$ for all $i, j$, and $Q=0$, a contradiction.

Let

$$
h(m, n)=\ell\left(R / H^{0}\left(W, \mathcal{O}_{W}\left(-m C_{0}-n D\right)\right)\right)
$$

be the function of (6). By the local Riemann-Roch formula (6), there exists a quadratic polynomial $A(m, n)$ such that

$$
\begin{aligned}
h(m, n) & =\ell\left(R / H^{0}\left(W, \mathcal{O}_{W}\left(-m C_{0}-n D\right)\right)\right) \\
& =A(m, n)-h^{1}\left(W, \mathcal{O}_{W}\left(-m C_{0}-n D\right)\right) \text { if } m, n \geq 0 .
\end{aligned}
$$

Thus the series (35)

$$
\sum_{m, n \in \mathbf{N}} h(m, n) s^{m} t^{n}
$$

is not rational.

\section{IRRATIONALITY IN CHARACTERISTIC ZERO}

In this section, we construct an example of a resolution $X \rightarrow \operatorname{spec}(R)$ where $\mathbf{k}=R / m$ has characteristic zero, there are 3 exceptional components, and the associated Poincaré series is not rational.

Theorem 9.1. There exists a two-dimensional complete normal local ring $R$, containing a field of characteristic 0 , and a resolution of singularities $g: X \rightarrow \operatorname{spec}(R)$ with three exceptional divisors $C, F_{1}$ and $F_{2}$ such that the series

$$
\sum_{n_{1}, n_{2}, n_{3} \in \mathbf{N}} h^{1}\left(\mathcal{O}_{X}\left(-n_{1} C-n_{2} F_{1}-n_{3} F_{2}\right)\right) t_{1}^{n_{1}} t_{2}^{n_{2}} t_{3}^{n_{3}}
$$

and

$$
\sum_{n_{1}, n_{2}, n_{3} \in \mathbf{N}} \ell\left(R / \Gamma\left(\mathcal{O}_{X}\left(-n_{1} C-n_{2} F_{1}-n_{3} F_{2}\right)\right)\right) t_{1}^{n_{1}} t_{2}^{n_{2}} t_{3}^{n_{3}}
$$

are not rational series.

Lemma 9.2. Suppose that $X$ is a nonsingular projective surface over $\mathbf{C}$, and $F_{1}, \ldots, F_{r}$ are integral analytically irreducible closed curves contained in $X$ such that $\left(F_{i} \cdot F_{j}\right)$ is negative definite. Let $F=F_{1}+\cdots+F_{r}$. Suppose that $F$ is connected. Then there exist a complete normal local ring $\hat{A}$ with maximal ideal $m$, and a projective $\hat{A}$ scheme $\pi: Y \rightarrow \operatorname{spec}(\hat{A})$ such that $\pi$ is birational, and an isomorphism away from $m, \pi^{-1}(m)_{\text {red }} \cong F$, and the formal schemes $Y_{F}$ and $X_{F}$ are isomorphic. 
Proof. By Grauert's contraction theorem [10] there exist a neighborhood $S$ of $F$ (in the complex topology), a normal analytic space $U$, and a bimeromorphic map $\lambda: S \rightarrow U$ such that $F$ is the exceptional locus of $\lambda$. Let $q \in U$ be the point such that $\lambda(F)=q, A=\mathcal{O}_{U, q}^{h}$ with maximal ideal $n$. Since the $F_{i}$ are analytically irreducible of dimension 1 , there exist an effective divisor $D$ on $X$ whose support is $F$, an ideal sheaf $\mathcal{J}$ on $X$ such that the support of $\mathcal{O}_{X} / \mathcal{J}$ is 0-dimensional, and if $\mathcal{I}=\mathcal{O}_{X}(-D) \mathcal{J}$, then $n \mathcal{O}_{S}^{h}=\mathcal{I} \mathcal{O}_{S}^{h}$. Let $\hat{A}$ be the $n$-adic completion of $A, m=n \hat{A}$.

Let $V_{1}, \ldots, V_{t}$ be an affine cover of $F$ in $X, R_{j}=\Gamma\left(V_{j}, \mathcal{O}_{X}\right), I_{j}=\Gamma\left(V_{j}, \mathcal{I}\right)$ for $1 \leq j \leq t$. We have compatible $\mathbf{C}$-algebra homomorphisms $A / n^{i} \rightarrow R_{j} / I_{j}^{i}$ for all $i$ and $j$, defined by the composition

$$
A / n^{i} \rightarrow \Gamma\left(S, \mathcal{O}_{S}^{h} / \mathcal{I}^{i} \mathcal{O}_{S}^{h}\right)=\Gamma\left(X, \mathcal{O}_{X} / \mathcal{I}^{i}\right) \rightarrow \Gamma\left(V_{j}, \mathcal{O}_{X} / \mathcal{I}^{i}\right) .
$$

The middle equality is by [27]. We thus have homomorphisms $\hat{A} \rightarrow \hat{R}_{j}$, where $\hat{R}_{j}$ is the $I_{j}$-adic completion of $R_{j}$. Each $\hat{R}_{j}$ is a domain (since $R_{j}$ is regular and excellent). We have isomorphisms

$$
\hat{A} \cong A\left[\left[x_{1}, \ldots, x_{n}\right]\right] /\left(x_{1}-a_{1}, \ldots, x_{s}-a_{s}\right)
$$

and

$$
\hat{R}_{j} \cong R_{j}\left[\left[x_{1}, \ldots, x_{n}\right]\right] /\left(x_{1}-a_{1}, \ldots, x_{s}-a_{s}\right),
$$

where $\left(a_{1}, \ldots, a_{s}\right)$ is a basis of $n$.

Since $R_{j}$ is of finite type over $\mathbf{C}, \hat{R}_{j}$ is of finite type over $\hat{A}$. Since $F$ is connected, we have an integral scheme $Y=\bigcup \operatorname{spec}\left(\hat{R}_{j}\right)$ of finite type over $\operatorname{spec}(\hat{A})$, with morphism $\pi: Y \rightarrow \operatorname{spec}(\hat{A})$ such that $\mathcal{O}_{Y} / m^{i} \mathcal{O}_{Y} \cong \mathcal{O}_{X} / \mathcal{I}^{i}$ for all $i$. By the valuative criterion for properness, $Y$ is proper over $\operatorname{spec}(\hat{A})$, since $F$ is proper over C, and $\hat{A}$ has dimension 2 .

Now we see by Grauert's comparison theorem (cf. Chapter III, Section 3 of [4]), [27] and the theorem on formal functions (12], III, section 4) that

$$
\hat{A}=\lim _{\leftarrow} \Gamma\left(S, \mathcal{O}_{S}^{h} / \mathcal{I}^{k} \mathcal{O}_{S}^{h}\right)=\lim _{\leftarrow} \Gamma\left(X, \mathcal{O}_{X} / \mathcal{I}^{k}\right)=\Gamma\left(Y, \mathcal{O}_{Y}\right)=\bigcap \hat{R}_{j}
$$

is integrally closed in the function field $\mathbf{C}(Y)$ of $Y$. Since $Y$ and $\hat{A}$ have dimension 2, the function field $\mathbf{C}(Y)$ of $Y$ is finite over the quotient field $Q(\hat{A})$, so that $Q(\hat{A})=\mathbf{C}(Y)$. By Zariski's Main Theorem, $\pi: Y \rightarrow \operatorname{spec}(\hat{A})$ is an isomorphism away from $m$, and $\pi^{-1}(m)_{\text {red }} \cong F$.

Finally, we will show that $\pi$ is projective. There exists an effective divisor $D=$ $\sum b_{i} F_{i}$ with support $F$ such that

$$
\left(-D \cdot F_{i}\right)>0 \text { for } 1 \leq i \leq r .
$$

Thus there exists $n_{0}$ such that $\mathcal{O}_{D}(-n D)$ is very ample and

$$
H^{1}\left(D, \mathcal{O}_{D}(-n D)\right)=0 \text { if } n \geq n_{0} .
$$

From the exact sequence

$$
0 \rightarrow \mathcal{O}_{D}(-n D) \rightarrow \mathcal{O}_{(n+1) D} \rightarrow \mathcal{O}_{n D} \rightarrow 0
$$

we conclude that

$$
H^{1}\left(X, \mathcal{O}_{Y}(-l D)\right)=\lim _{\leftarrow} H^{1}\left(Y, \mathcal{O}_{n D}(-l D)\right)=0 \text { if } l \geq n_{0} .
$$


We thus have a surjection

$$
H^{0}\left(Y, \mathcal{O}_{Y}(-n D)\right) \rightarrow H^{0}\left(D, \mathcal{O}_{D}(-n D)\right)
$$

if $n \geq l$. In particular, $K_{a}=H^{0}\left(Y, \mathcal{O}_{Y}(-a D)\right)$ is generated by global sections if $a \geq l$, so that $\bigoplus_{n \geq 0} K_{n}$ is a finitely generated $\hat{A}$ algebra (cf. Proposition III.3.3.1 in 12]). Thus there exists $t \geq l$ such that $\bigoplus_{n>0} K_{t n}$ is generated in degree 1 , so that $K_{t n}=K_{t}^{n}$ for all $n \geq 1$. If $\nu_{i}$ is the discrete valuation with valuation ring $\mathcal{O}_{Y, F_{i}}$, then

$$
K_{a}=\left\{f \in \hat{A} \mid \nu_{i}(f) \geq a b_{i} \text { for } 1 \leq i \leq r\right\},
$$

so that each $K_{a}$ is an intersection of valuation ideals, and is thus integrally closed. Thus $\bigoplus_{n \geq 0} K_{t}^{n}$ is a normal ring, and $Z=\operatorname{proj}\left(\bigoplus_{n \geq 0} K_{t}^{n}\right)$ is a normal scheme. Since $K_{t} \mathcal{O}_{Y}=\mathcal{O}_{Y}(-t D)$ is invertible, we have a birational morphism $\tau: Y \rightarrow Z$ which is an isomorphism away from $F$. As $K_{t} \mathcal{O}_{D}$ is very ample, $\tau$ does not contract any component of $F$. By Zariski's Main Theorem, $\tau$ is an isomorphism.

Lemma 9.3. There exists a rational, complex Gorenstein projective curve $C$ which has an isolated singularity $\tilde{p}$, with local ring

$$
\mathcal{O}_{C, \tilde{p}} \cong \mathbf{C}\left[t^{2}, t^{5}\right]_{\left(t^{2}, t^{5}\right)}
$$

and the following properties:

(1) $C$ has arithmetic genus $p_{a}(C)=2$.

(2) If $\mathcal{L}$ is a line bundle on $C$ and $\operatorname{deg}(\mathcal{L}) \geq 8$, then $\mathcal{L}$ is generated by global sections.

(3) If $\mathcal{L}$ is a line bundle on $C$ and $\operatorname{deg}(\mathcal{L}) \geq 10$, then $\mathcal{L}$ is very ample.

(4) If $\mathcal{L}$ is a line bundle on $C$ of negative degree, there exist a nonsingular projective surface $S$ and an embedding $C \subset S$ such that $C \cdot C \sim \mathcal{L}$.

Proof. Let $C_{0}=V\left(x_{2}^{3} x_{1}^{2}-x_{0}^{5}\right) \subset X_{0}=\mathbf{P}_{\mathbf{C}}^{2}$. $C_{0}$ is a rational curve with two singular points, $q_{0}=(0: 0: 1)$ and $q_{1}=(0: 1: 0)$. We will resolve the singularity at $q_{1}$. There are regular parameters $y=\frac{x_{0}}{x_{1}}, z=\frac{x_{2}}{x_{1}}$ at $q_{1}$, and

$$
\mathcal{O}_{C_{0}, q_{1}}=\left(\mathbf{C}[y, z] / z^{3}-y^{5}\right)_{(y, z)} .
$$

Let $\pi_{1}: X_{1} \rightarrow X_{0}$ be the blowup of $q_{1}$. Let $C_{1}$ be the strict transform of $C_{0}$ on $X_{1}$. Let $E_{1}=\pi_{1}^{-1}\left(q_{1}\right), q_{2}=E_{1} \cap C_{1}$. Let $\left(y_{1}, z_{1}\right)$ be the regular parameters at $q_{2}$ defined by $y=y_{1}, z=y_{1} z_{1}$. Then $z_{1}^{3}-y_{1}^{2}=0$ is a local equation of $C_{1}$ at $q_{2}$. Let $\pi_{2}: X_{2} \rightarrow X_{1}$ be the blowup of $q_{2}$. Let $C_{2}$ be the strict transform of $C_{1}$ on $X_{2}$, and let $E_{2}=\pi_{1}^{-1}\left(q_{2}\right)$ and $q_{3}=E_{2} \cap C_{2}$. Let $\left(y_{2}, z_{2}\right)$ be the regular parameters at $q_{3}$ defined by $y_{1}=y_{2} z_{2}, z_{1}=z_{2}$. Then $z_{2}-y_{2}^{2}=0$ is a local equation of $C_{2}$ at $q_{3}$, and $C_{2}$ is thus nonsingular at $q_{3}$. Set $\pi=\pi_{1} \circ \pi_{2}$. Identify $E_{1}$ with its strict transform on $X_{2} . q_{0}$ is the only singular point on $C_{2}$. We have

$$
\begin{gathered}
\pi_{1}^{*}\left(C_{0}\right)=C_{1}+3 E_{1} \cdot \pi^{*}\left(C_{0}\right)=C_{2}+5 E_{2}+3 E_{1}, \\
C_{2} \cdot C_{2} \sim \pi^{*}\left(C_{0}\right) \cdot C_{2}-5 E_{2} \cdot C_{2}-3 E_{1} \cdot C_{2} .
\end{gathered}
$$

$C_{2} \cdot E_{2}$ and $C_{2} \cdot E_{1}$ are supported at $q_{2}, y_{2}=0$ is a local equation of $E_{1}$ at $q_{2}$, and $z_{2}=0$ is a local equation of $E_{2}$ at $q_{2}$. Thus $C_{2} \cdot E_{1}=q_{2}, C_{2} \cdot E_{2}=2 q_{2}$, and

$$
C_{2} \cdot C_{2} \sim \pi^{*}\left(C_{0}\right) \cdot C_{2}-13 q_{2} \text {. }
$$


Let $V$ be a general quintic curve on $X_{0}$. We have

$$
V \cdot C_{0}=p_{1}+\cdots+p_{25}
$$

where $p_{1}, \ldots, p_{25} \in C_{0}$ are distinct nonsingular points. Also,

$$
\pi^{*}\left(C_{0}\right) \cdot C_{2} \sim \pi^{*}(V) \cdot C_{2}=p_{1}+\cdots+p_{25} .
$$

Thus

$$
C_{2} \cdot C_{2} \sim p_{1}+\cdots+p_{25}-13 q_{2}
$$

Set $D=C_{2} \cdot C_{2}$. We have that $\operatorname{deg}(D)=12$,

$$
\hat{\mathcal{O}}_{C_{2}, q_{0}}=\mathbf{C}\left[\left[\frac{x_{0}}{x_{2}}, \frac{x_{1}}{x_{2}}\right]\right] /\left(\frac{x_{1}}{x_{2}}\right)^{2}-\left(\frac{x_{0}}{x_{2}}\right)^{5} \cong \mathbf{C}\left[\left[t^{2}, t^{5}\right]\right] \subset \mathbf{C}[[t]]
$$

and $\ell\left(\mathbf{C}[[t]] / \hat{\mathcal{O}}_{C_{2}, q_{0}}\right)=2$. Let $C=C_{2}$.

The arithmetic genus of $C$ is thus

$$
p_{a}(C)=p_{a}\left(\mathbf{P}^{1}\right)+2=2
$$

(cf. Exercise IV 1.8 [14]).

Since $C$ is a local complete intersection, the Riemann-Roch theorem is applicable on $C$ (cf. Exercise IV. 1.9 in [14]). In particular, there is a canonical bundle $\omega_{C}$ on $C$ such that for any line bundle $\mathcal{L}$ on $C, h^{1}(C, \mathcal{L})=h^{0}\left(C, \omega_{C} \otimes \mathcal{L}^{-1}\right)$ (Serre duality) and $\chi(\mathcal{L})=\operatorname{deg}(\mathcal{L})+1-p_{a}(C)$. We further have $\operatorname{deg}\left(\omega_{C}\right)=2 p_{a}(C)-2=2$.

$C$ has an affine cover by open sets $U_{1}=\operatorname{spec}\left(\mathbf{C}\left[t^{2}, t^{5}\right]\right)$ and $U_{2}=\operatorname{spec}\left(\mathbf{C}\left[\frac{1}{t}\right]\right)$. Let $\infty$ be the point on $C$ with maximal ideal $\left(\frac{1}{t}\right)$. $\infty$ is the point $q_{3}$ on $C_{2}$. Let $\tilde{p}$ be the point $q_{0}$ on $C$. Let $m$ be the the ideal sheaf of the point $\tilde{p}$. Then $\left.\Gamma\left(U_{1}, m\right)=\left(t^{2}, t^{5}\right) \subset \mathbf{C}\left[t^{2}, t^{5}\right]\right)$. Consider the line bundle $\mathcal{L}_{1}$ defined by

$$
\begin{aligned}
& H^{0}\left(U_{1}, \mathcal{L}_{1}\right)=\mathbf{C}\left[t^{2}, t^{5}\right] t^{2}, \\
& H^{0}\left(U_{2}, \mathcal{L}_{1}\right)=\mathbf{C}\left[\frac{1}{t}\right],
\end{aligned}
$$

and the line bundle $\mathcal{L}_{2}$ defined by

$$
\begin{aligned}
& H^{0}\left(U_{1}, \mathcal{L}_{2}\right)=\mathbf{C}\left[t^{2}, t^{5}\right] t^{5}, \\
& H^{0}\left(U_{2}, \mathcal{L}_{2}\right)=\mathbf{C}\left[\frac{1}{t}\right] .
\end{aligned}
$$

Multiplying $\mathcal{L}_{1}$ by $\frac{1}{t^{2}}$ and $\mathcal{L}_{2}$ by $\frac{1}{t^{5}}$, we see that $\mathcal{L}_{1} \cong \mathcal{O}_{C}(-2 \infty)$ and $\mathcal{L}_{2} \cong$ $\mathcal{O}_{C}(-5 \infty)$. We thus have short exact sequences

$$
\begin{aligned}
& 0 \rightarrow \mathcal{K}_{1} \rightarrow \mathcal{O}_{C}(-2 \infty) \oplus \mathcal{O}_{C}(-5 \infty) \rightarrow m \rightarrow 0, \\
& 0 \rightarrow \mathcal{K}_{2} \rightarrow \mathcal{O}_{C}(-4 \infty) \oplus \mathcal{O}_{C}(-7 \infty) \rightarrow m^{2} \rightarrow 0
\end{aligned}
$$

of coherent $\mathcal{O}_{C}$ modules, for some modules $\mathcal{K}_{1}$ and $\mathcal{K}_{2}$.

Let $a \in C$ be a closed point, with ideal sheaf $m_{a}$, and let $\mathcal{L}$ be a line bundle on $C$. From the exact sequence

$$
0 \rightarrow \mathcal{L} m_{a} \rightarrow \mathcal{L} \rightarrow \mathcal{L} / \mathcal{L} m_{a} \rightarrow 0
$$

we see that $\mathcal{L}$ is generated by global sections if $H^{1}\left(C, \mathcal{L} m_{a}\right)=0$ for all $a \in C$. By Serre duality, we have that if $\mathcal{N}$ is a line bundle of degree $>2$, then $H^{1}(C, \mathcal{N})=0$. By (39) we see that $\mathcal{L}$ is generated by global sections if $\operatorname{deg}(\mathcal{L}) \geq 8$.

By Proposition II.7.3 in [14], a line bundle $\mathcal{L}$ on $C$ is very ample if

(1) $\mathcal{L}$ is generated by global sections,

(2) $H^{0}\left(C, \mathcal{L} m_{a}\right) \rightarrow H^{0}\left(C, \mathcal{L} m_{a} / \mathcal{L} m_{a} m_{b}\right)$ is surjective for distinct closed points $a, b \in C$, and 
(3) $H^{0}\left(C, \mathcal{L} m_{a}\right) \rightarrow H^{0}\left(C, \mathcal{L} m_{a} / \mathcal{L} m_{a}^{2}\right)$ is surjective for every closed point $a \in C$.

From the exact sequences

$$
0 \rightarrow \mathcal{L} m_{a} m_{b} \rightarrow \mathcal{L} m_{a} \rightarrow \mathcal{L} m_{a} / \mathcal{L} m_{a} m_{b} \rightarrow 0
$$

and

$$
0 \rightarrow \mathcal{L} m_{a}^{2} \rightarrow \mathcal{L} m_{a} \rightarrow \mathcal{L} m_{a} / \mathcal{L} m_{a}^{2} \rightarrow 0,
$$

Serre duality, (39) and (40), we see that $\mathcal{L}$ is very ample if $\operatorname{deg}(\mathcal{L}) \geq 10$.

Suppose that $\mathcal{L} \in \operatorname{Pic}\left(C_{2}\right)$ has degree $-e<0$. Let $r=e+12$. Then $\operatorname{deg}(D-\mathcal{L})=$ $r>10$ implies $D-\mathcal{L}$ is very ample, so by Bertini's theorem,

$$
D-\mathcal{L} \sim a_{1}+\cdots+a_{r},
$$

where $a_{1}, \ldots, a_{r} \in C_{2}$ are distinct nonsingular points in $C_{2}$. Let $\lambda: X_{3} \rightarrow X_{2}$ be the blowup of $a_{1}, \ldots, a_{r}$. Let $F_{i}=\lambda^{-1}\left(a_{i}\right)$ for $1 \leq i \leq r$. Let $C_{3} \cong C$ be the strict transform of $C_{2}$. Then $\lambda^{*}\left(C_{2}\right)=C_{3}+F_{1}+\cdots+F_{r}$, and

$$
C_{3} \cdot C_{3} \sim \lambda^{*}\left(C_{2}\right) \cdot C_{3}-a_{1}-\cdots-a_{r} \sim D-a_{1}-\cdots-a_{r} \sim \mathcal{L} .
$$

Let $C$ be the curve of Lemma 9.3 , with singular point $\tilde{p}$. Let $\pi: \mathbf{P}^{1} \rightarrow C$ be the normalization of $C$, with function field $\mathbf{C}\left(\mathbf{P}^{1}\right)=\mathbf{C}(t)$, where $t=0$ is a local equation of $q=\pi^{-1}(\tilde{p})$. Let $\infty \in C$ be the point with local equation $\frac{1}{t}=0$. Let

$$
\begin{gathered}
\operatorname{Div}^{0}=\text { group of Weil divisors of degree } 0 \text { on } C-\tilde{p} . \\
\qquad \operatorname{Pic}^{0}(C) \cong \operatorname{Div}^{0} / \sim,
\end{gathered}
$$

where $D_{1} \sim D_{2}$ if $D_{1}-D_{2}=(f)$ for some $f \in \mathbf{C}(t)$ which is a unit in $\mathcal{O}_{C, \tilde{p}}$ (cf. II.6 in [14]).

Suppose that $D \in \operatorname{Div}^{0}$. There exists $f_{D} \in \mathbf{C}(t)$ such that $\left(f_{D}\right)=D$ (divisor computed on $\mathbf{P}^{1}$ ), and $f_{D}$ is unique up to multiplication by a nonzero constant in C. Define $\Lambda: \operatorname{Div}^{0} \rightarrow \mathbf{C}^{2}$ by

$$
\Lambda(D)=\left(\left.\frac{d}{d t} \log \left(f_{D}\right)\right|_{t=0},\left.\frac{d^{3}}{d t^{3}} \log \left(f_{D}\right)\right|_{t=0}\right) .
$$

$\Lambda(D)$ is a well defined group homomorphism.

For $D \in \operatorname{Div}^{0}$, we have an expansion

$$
f_{D}=\sum_{i=0}^{\infty} a_{i} t^{i} \in \hat{\mathcal{O}}_{\mathbf{P}^{1}, q}=\mathbf{C}[[t]]
$$

where $a_{0} \neq 0$. We have $f_{D} \in \hat{\mathcal{O}}_{C, \tilde{p}}$ if and only if $a_{1}=a_{3}=0$. Since $f_{D} \in \mathbf{C}(t)$ and $\mathcal{O}_{C, \tilde{p}}=\hat{\mathcal{O}}_{C, \tilde{p}} \cap \mathbf{C}(t)$ (cf. Lemma 2 in [1]), $f_{D} \in \mathcal{O}_{C, \tilde{p}}$ if and only if $a_{1}=a_{3}=0$. Since $\Lambda(D)=0$ if and only if $a_{1}=a_{3}=0$, we have $\Lambda(D)=0$ if and only if $D \sim 0$. By allowing $a_{1}$ and $a_{3}$ to vary, we see that $\Lambda$ is onto. Thus $\Lambda$ is a group isomorphism of $\operatorname{Pic}^{0}(C)$ with $\mathbf{C}^{2}$.

We will consider the Abel-Jacobi map

$$
A J: C-\{\tilde{p}\} \rightarrow \mathbf{C}^{2}
$$

defined by $A J(\alpha)=\Lambda(\alpha-\infty)$. We have

$$
A J(\alpha)=\left(\left.\frac{d \log (t-\alpha)}{d t}\right|_{t=0},\left.\frac{d^{3} \log (t-\alpha)}{d t^{3}}\right|_{t=0}\right)=\left(\frac{1}{-\alpha}, \frac{2}{-\alpha^{3}}\right) .
$$


Define the image of the Abel-Jacobi map to be

$$
W=A J(C-\{\tilde{p}\}) .
$$

$W$ is the subvariety of $\mathbf{C}^{2}$ defined by $y=2 x^{3}$.

Lemma 9.4. Suppose that $D \in D i v^{0}$. Then

$$
h^{1}\left(C, \mathcal{O}_{C}(D+\infty)\right)=h^{0}\left(C, \mathcal{O}_{C}(D+\infty)\right)= \begin{cases}0 & \text { if } \Lambda(D) \notin W \\ 1 & \text { if } \Lambda(D) \in W\end{cases}
$$

Proof. There exists $f \in \mathbf{C}(t)$ such that $(f)=D$ (divisor computed on $\mathbf{P}^{1}$ ). Then

$$
H^{0}\left(\mathbf{P}^{1}, \mathcal{O}_{\mathbf{P}^{1}}(D+\infty)\right)
$$

has $\mathbf{C}$ basis $\frac{1}{f}, \frac{t}{f}$, and

$$
H^{0}\left(C, \mathcal{O}_{C}(D+\infty)\right)=\left\{\lambda \in H^{0}\left(\mathbf{P}^{1}, \mathcal{O}_{\mathbf{P}^{1}}(D+\infty)\right) \mid \lambda \in \mathcal{O}_{C, \tilde{p}}\right\} .
$$

There is an expansion

$$
\frac{t}{f}=a_{1} t+a_{2} t^{2}+\cdots
$$

in $\mathbf{C}[[t]]=\hat{\mathcal{O}}_{\mathbf{P}^{1}, q}$, where $a_{1} \neq 0$, so $\frac{t}{f} \notin \mathcal{O}_{C, \tilde{p}}$. For $0 \neq \alpha \in \mathbf{C}$,

$$
\begin{aligned}
\frac{t-\alpha}{f} \in \mathcal{O}_{C, \tilde{p}} & \Leftrightarrow \Lambda(-D+\alpha-\infty)=0 \\
& \Leftrightarrow \Lambda(D)=A J(\alpha) .
\end{aligned}
$$

We further have

$$
\frac{1}{f} \in \mathcal{O}_{C, \tilde{p}} \Leftrightarrow \Lambda(-D)=0 \Leftrightarrow \Lambda(D)=A J(\infty)=0 .
$$

Since $p_{a}(C)=2$, the equality of $h^{0}$ and $h^{1}$ follows from the Riemann-Roch theorem.

Suppose that $\mathcal{M}$ is a line bundle on $C$ of degree $-d$ with $d \geq 3$, and $p_{1}, p_{2}$ are distinct points on $C-\{\tilde{p}\}$. Let $\overline{\mathcal{L}}=\mathcal{M} \otimes \mathcal{O}_{C}\left(p_{1}+p_{2}\right)$. Lemma 9.3 shows that there exists a nonsingular surface $S$ and an embedding $C \subset S$ such that $C \cdot C=\overline{\mathcal{L}}$. Also, $\operatorname{deg}(\overline{\mathcal{L}})<0$ and $C$ is a local complete intersection in $S$, so by Lemma 9.2 , there is a birational (projective) morphism $\pi: Y \rightarrow \operatorname{spec}(R)$, where $R$ is a complete normal local ring of dimension 2, with exceptional divisor $C$ such that $Y$ is nonsingular, $C \cdot C=\overline{\mathcal{L}}$.

Let $X \rightarrow Y$ be the blowup of $Y$ at the points $p_{1}$ and $p_{2}$, with exceptional divisors $F_{1}$ and $F_{2}$ (both isomorphic to $\mathbf{P}^{1}$ ). Identify $C$ with its strict transform on $X$. Then $X \rightarrow \operatorname{spec}(R)$ is a resolution with exceptional curves $F_{1}, F_{2}$ and $C, C \cdot C \sim \mathcal{M}$, $C \cdot F_{1}=p_{1}, C \cdot F_{2}=p_{2},\left(F_{1}^{2}\right)=-1,\left(F_{2}^{2}\right)=-1$.

Lemma 9.5. Suppose that $\mathcal{N}$ is a line bundle on $X$ such that

$$
(\mathcal{N} \cdot C)>4-d,\left(\mathcal{N} \cdot F_{1}\right) \geq 0,\left(\mathcal{N} \cdot F_{2}\right) \geq 0
$$

or

$$
(\mathcal{N} \cdot C)=4-d, \mathcal{N} \cdot C \not \omega_{C}+\overline{\mathcal{L}},\left(\mathcal{N} \cdot F_{1}\right) \geq 0,\left(\mathcal{N} \cdot F_{2}\right) \geq 0 .
$$

Then $H^{1}(X, \mathcal{N}) \cong H^{1}\left(C, \mathcal{O}_{C} \otimes \mathcal{N}\right)$. 
Proof. We have exact sequences

$$
\begin{aligned}
& 0 \rightarrow \mathcal{O}_{F_{1}+F_{2}}\left(-n\left(F_{1}+F_{2}+C\right)-C\right) \otimes \mathcal{N} \rightarrow \mathcal{O}_{(n+1)\left(F_{1}+F_{2}+C\right)} \otimes \mathcal{N} \\
& \rightarrow \mathcal{O}_{n\left(F_{1}+F_{2}+C\right)+C} \otimes \mathcal{N} \rightarrow 0
\end{aligned}
$$

for $n \geq 0$, and

$0 \rightarrow \mathcal{O}_{C}\left(-n\left(F_{1}+F_{2}+C\right)\right) \otimes \mathcal{N} \rightarrow \mathcal{O}_{n\left(F_{1}+F_{2}+C\right)+C} \otimes \mathcal{N} \rightarrow \mathcal{O}_{n\left(F_{1}+F_{2}+C\right)} \otimes \mathcal{N} \rightarrow 0$

for $n \geq 1$. Moreover,

$$
\begin{aligned}
\left(C \cdot\left(-n\left(F_{1}+F_{2}+C\right)\right)+(C \cdot \mathcal{N})\right. & =n(d-2)+(C \cdot \mathcal{N}) \\
& \geq(d-2)+(C \cdot \mathcal{N}) \geq 2 p_{a}(C)-2=2
\end{aligned}
$$

and

$$
\left(F_{i} \cdot\left(-n\left(F_{1}+F_{2}+C\right)-C\right)\right)+\left(\mathcal{N} \cdot F_{i}\right)=-1+\left(\mathcal{N} \cdot F_{i}\right) \geq-1
$$

for $i=1,2$. Thus

$$
H^{1}\left(X, \mathcal{O}_{n\left(F_{1}+F_{2}+C\right)} \otimes \mathcal{N}\right) \cong H^{1}\left(C, \mathcal{O}_{C} \otimes \mathcal{N}\right)
$$

for all $n>0$, and

$$
\left.H^{1}(X, \mathcal{N}) \cong \lim _{\leftarrow} H^{1}\left(X, \mathcal{O}_{n\left(F_{1}+F_{2}+C\right)}\right) \otimes \mathcal{N}\right) \cong H^{1}\left(C, \mathcal{O}_{C} \otimes \mathcal{N}\right)
$$

We will now fix $d=3$, and continue to assume that $\mathcal{M}$ is a line bundle of degree $-d=-3$ on $C$. Let $\mathcal{L}=\mathcal{M}+3 \infty$. Set

$$
\begin{aligned}
& \Lambda_{1}=-2 F_{1}-F_{2}-C, \\
& \Lambda_{2}=-F_{1}-2 F_{2}-C, \\
& \Lambda_{3}=-F_{1}-F_{2}-C .
\end{aligned}
$$

$\Lambda_{1}, \Lambda_{2}, \Lambda_{3}$ generate the integral lattice $\mathbf{Z}^{3}$ in $\mathbf{R} F_{1}+\mathbf{R} F_{2}+\mathbf{R} C$, and if $C=F_{3}$, then $\left(\Lambda_{i} \cdot F_{j}\right)=\delta_{i j}$ for $1 \leq i, j \leq 3$. Set $\bar{\Delta}_{i}=\Lambda_{i} \cdot C$ for $1 \leq i \leq 3$. For a point $p \in C-\tilde{p}$, set $\bar{p}=p-\infty$. Then

$$
\begin{aligned}
& \bar{\Delta}_{1}=-2 p_{1}-p_{2}-\mathcal{M} \quad \sim-2 \bar{p}_{1}-\bar{p}_{2}-\mathcal{L} \\
& \bar{\Delta}_{2}=-p_{1}-2 p_{2}-\mathcal{M} \quad \sim-\bar{p}_{1}-2 \bar{p}_{2}-\mathcal{L}
\end{aligned}
$$

Now we will fix $p_{1}, p_{2}$ and $\mathcal{M}$ so that $\bar{p}_{1}=(0,0), \bar{p}_{2}=(-1,-2), \mathcal{L}=(1,1)$. Then $\bar{\Delta}_{1}=(0,1), \bar{\Delta}_{2}=(1,3)$. By Lemma 9.4, for $m, n \geq 0$

$$
h^{1}\left(C, \mathcal{O}_{C}\left(m \bar{\Delta}_{1}+n \bar{\Delta}_{2}+F_{1}\right)\right)= \begin{cases}1 & m=2 n^{3}-3 n \\ 0 & \text { otherwise. }\end{cases}
$$

Lemma 9.6. There exists at most one divisor $D=-a F_{1}-b F_{2}-c C$ on $X$ such that $D \cdot C \sim \omega_{C}$.

Proof. Suppose that $D \cdot C \sim \omega_{C}$. Then

$$
\begin{aligned}
\operatorname{deg} \omega_{C}= & 2 p_{a}(C)-2=2=(D \cdot C)=-a-b+3 c, \\
\omega_{C}-2 \infty & \sim-a \bar{p}_{1}-b \bar{p}_{2}-c \mathcal{L} \\
& \sim-b(-1,-2)-c(1,1)=(b-c, 2 b-c),
\end{aligned}
$$

which implies that there exists at most one value of $(b, c)$ such that $D \cdot C \sim \omega_{C}$. Since $a=3 c-b-2$, there is at most one value of $(a, b, c)$. 
Lemma 9.7. There exists a number $\lambda$ such that $a+b+c>\lambda$ and $a, b, c>0$ imply

$$
h^{1}\left(X, \mathcal{O}_{X}\left(a \Lambda_{1}+b \Lambda_{2}+c \Lambda_{3}\right)\right)= \begin{cases}0 & \text { if } c \geq 2, \\ 1 & \text { if } c=1, a=2 b^{3}-3 b-1, \\ 0 & \text { if } c=1, a \neq 2 b^{3}-3 b-1 .\end{cases}
$$

Proof. By Lemma 9.6 there exists $\lambda>0$ such that $a, b, c>0$ and $a+b+c>\lambda$ imply

$$
\left(a \Lambda_{1}+b \Lambda_{2}+c \Lambda_{3}\right) \cdot C \not \omega_{C}+\left(F_{1}+F_{2}+C\right) \cdot C \sim \omega_{C}+\overline{\mathcal{L}}
$$

and

$$
\left(a \Lambda_{1}+b \Lambda_{2}+c \Lambda_{3}\right) \cdot C \nsim \omega_{C} .
$$

By Lemma $9.5 a, b>0, c \geq 2$ and $a+b+c>\lambda$ imply

$$
H^{1}\left(X, \mathcal{O}_{X}\left(a \Lambda_{1}+b \Lambda_{2}+c \Lambda_{3}\right)\right) \cong H^{1}\left(C, \mathcal{O}_{C}\left(a \bar{\Delta}_{1}+b \bar{\Delta}_{2}+c \bar{\Delta}_{3}\right)\right)=0
$$

since $\left(C \cdot\left(a \Lambda_{1}+b \Lambda_{2}+c \Lambda_{3}\right)\right) \geq 2=2 p_{a}(C)-2$. By Lemma 9.5,

$$
\begin{aligned}
H^{1}\left(X, \mathcal{O}_{X}\left(a \Lambda_{1}+b \Lambda_{2}+\Lambda_{3}\right)\right) & \cong H^{1}\left(C, \mathcal{O}_{C}\left(a \bar{\Delta}_{1}+b \bar{\Delta}_{2}+\bar{\Delta}_{3}\right)\right) \\
& =H^{1}\left(C, \mathcal{O}_{C}\left((a+1) \bar{\Delta}_{1}+b \bar{\Delta}_{2}+\infty\right)\right),
\end{aligned}
$$

since

$$
a \Lambda_{1}+b \Lambda_{2}+\Lambda_{3}=(a+1) \Lambda_{1}+b \Lambda_{2}+F_{1},
$$

and Lemma 9.7 follows from (43).

We will now give the proof of Theorem 9.1. Set $R=\hat{A}$, and let the notation be as above for the surface $X$. Set $a_{i j k}=h^{1}\left(\mathcal{O}_{X}\left(-i F_{1}-j F_{2}-k C\right)\right)$. We will first show that the series

$$
f=\sum_{i, j, k=0}^{\infty} a_{i j k} t_{1}^{i} t_{2}^{j} t_{3}^{k}
$$

of (37) is not rational.

Suppose that $f$ is rational. Then there exists a nonzero polynomial

$$
Q=\sum_{i, j, k=0}^{r} b_{i j k} t_{1}^{i} t_{2}^{j} t_{3}^{k}
$$

such that $f Q$ is a polynomial. Thus there is $\sigma>0$ such that

$$
\sum_{i, j, k=0}^{r} a_{l-i, m-j, n-k} b_{i, j, k}=0
$$

whenever $l+m+n \geq \sigma$ and $l, m, n \geq r$.

We will prove that $Q=0$, and derive a contradiction to the assumption that $f$ is rational, by induction on $0 \leq \alpha$ in the following statement:

If $i, j, k$ are such that $0 \leq i, j, k \leq r$ and $(3 r+1)+i+j-3 k<1+\alpha$, then $b_{i j k}=0$. 
The statement is vacuously true for $\alpha=0$, so we will assume it to be true for $\alpha$ and prove it for $\alpha+1$. Set

$$
\begin{aligned}
& l=2 a+b-1+\alpha, \\
& m=a+2 b, \\
& n=a+b+r,
\end{aligned}
$$

where $a, b$ are abitrary, subject to the conditions

$$
a \geq \max \{\sigma, \lambda+4 r\}, \quad b \geq \max \{\sigma, \lambda+4 r\},
$$

where $\lambda$ is the integer of Lemma 9.7. For $0 \leq i, j, k \leq r$, set

$$
D_{i j k}=-(l-i) F_{1}-(m-j) F_{2}-(n-k) C .
$$

We have $\left(D_{i j k} \cdot F_{1}\right) \geq 1,\left(D_{i j k} \cdot F_{2}\right) \geq 1$ and

$$
\left(D_{i j k} \cdot C\right)=(3 r+1)-\alpha+i+j-3 k .
$$

We have (by Lemma 9.7)

$$
a_{l-i, m-j, n-k}=0 \text { if }(3 r+1)-\alpha+i+j-3 k>1
$$

and (by assumption)

$$
b_{i j k}=0 \text { if }(3 r+1)-\alpha+i+j-3 k<1 .
$$

Thus

$$
0=\sum_{(3 r+1)-\alpha+i+j-3 k=1} a_{l-i, m-j, n-k} b_{i j k} .
$$

If $(3 r+1)-\alpha+i+j-3 k=1$, we have $\left(\left(D_{i j k}-F_{1}\right) \cdot C\right)=0$, so

$$
D_{i j k} \sim \beta \Lambda_{1}+\gamma \Lambda_{2}+F_{1}
$$

with

$$
\begin{aligned}
& \beta=(l-i+1)-(n-k)=a+\alpha-r-i+k, \\
& \gamma=(m-j)-(n-k)=b-r-j+k .
\end{aligned}
$$

For fixed $(a, b)$ and $(i, j)$ satisfying $0 \leq i, j \leq r$ and $(3 r+1)-\alpha+i+j+3 k=1$,

$$
h^{1}\left(X, \mathcal{O}_{X}\left(D_{i j k}\right)\right)= \begin{cases}0 & \text { if } \beta \neq 2 \gamma^{3}-3 \gamma-1, \\ 1 & \text { if } \beta=2 \gamma^{3}-3 \gamma-1,\end{cases}
$$

by Lemma 9.7 since

$$
D_{i j k} \sim(\beta-1) \Lambda_{1}+\gamma \Lambda_{2}+\Lambda_{3}
$$

We now observe that, given $n>0$, there exists $m(n)>0$ such that if $x_{0}>m(n)$ is an integer, and $y_{0}=2 x_{0}^{3}-3 x_{0}-1$, then

$$
\left(\left[x_{0}-n, x_{0}+n\right] \times\left[y_{0}-n, y_{0}+n\right]\right) \cap \mathbf{Z}^{2} \cap\left\{y=2 x^{3}-3 x-1\right\}=\left\{\left(x_{0}, y_{0}\right)\right\} .
$$

We can thus choose $(a, b)$ so that for any $\bar{i}, \bar{j}, \bar{k}$ such that $0 \leq \bar{i}, \bar{j}, \bar{k} \leq r$ satisfying $3 r+1-\alpha+\bar{i}+\bar{j}+3 \bar{k}=1$,

$$
0=\sum_{i, j, k=0}^{r} a_{l-i, m-j, n-k} b_{i j k}=b_{\overline{i j k}} .
$$

We thus conclude that the series of (37) is not rational, and by the local RiemannRoch theorem (6), (38) is not rational. 


\section{A nontrivial SEMI-PERIODIC $h^{1}$}

We give an example showing that $h^{1}$ is a nontrivial function.

Let $Z=\mathbf{P}^{2}$ be two-dimensional complex projective space. Let $C$ be a nonsingular cubic curve in $Z$.

Fix a point $p_{\infty} \in C$ as the 0 in the group law on $C$. For $p \in C$, let $\bar{p}$ be the divisor $p-p_{\infty}$. We then have a group isomorphism $C \rightarrow \operatorname{Pic}^{0}(C)$ given by $p \mapsto \bar{p}$. Let $p_{1}, p_{2} \in C$ be distinct points such that $\bar{p}_{1}=-\bar{p}_{2} \neq 0$.

By Bertini's theorem, there exists a cubic curve $V$ in $Z$ such that

$$
V \cdot C=Q_{1}+Q_{2}+\cdots+Q_{9},
$$

where $Q_{1}, \ldots, Q_{9}, p_{\infty}, p_{1}, p_{2}$ are distinct points. Let $\pi: X \rightarrow \mathbf{P}^{2}$ be the blowup of $Q_{1}, \ldots, Q_{9}, p_{1}, p_{2}, p_{\infty}$. Let $E_{1}=\pi^{-1}\left(p_{1}\right), E_{2}=\pi^{-1}\left(p_{2}\right), F_{i}=\pi^{-1}\left(Q_{i}\right)$, for $1 \leq i \leq 9, E_{\infty}=\pi^{-1}\left(p_{\infty}\right)$. Let $\bar{C}$ be the strict transform of $C$ on $X$. Then

$$
\pi^{*}(C) \cdot \bar{C} \sim V \cdot C=Q_{1}+\cdots+Q_{9}
$$

and $\pi^{*}(C)=\bar{C}+F_{1}+\cdots+F_{9}+E_{\infty}+E_{1}+E_{2}$, so that

$$
\bar{C} \cdot \bar{C} \sim-p_{\infty}-p_{1}-p_{2} .
$$

By Lemma 9.2, there exist a complete normal local ring $R$, and a birational morphism $\lambda: Y \rightarrow \operatorname{spec}(R)$, where the reduced exceptional fiber of $\lambda$ is $\bar{C}+E_{1}+E_{2}$ and the formal completion of $Y$ along $\bar{C}+E_{1}+E_{2}$ is isomorphic to the formal completion of $X$ along $\bar{C}+E_{1}+E_{2}$.

Let $\mathcal{L}=-\bar{C} \cdot \bar{C}-3 p_{\infty}$. Then

$$
\begin{aligned}
& \mathcal{L}=\bar{p}_{1}+\bar{p}_{2} \sim 0, \\
& \mathcal{L}-2 \bar{p}_{1}-\bar{p}_{2} \sim-\bar{p}_{1}, \\
& \mathcal{L}-\bar{p}_{1}-2 \bar{p}_{2} \sim-\bar{p}_{2} \sim \bar{p}_{1} .
\end{aligned}
$$

Set

$$
\begin{gathered}
\Lambda_{1}=-\bar{C}-2 E_{1}-E_{2}, \\
\Lambda_{2}=-\bar{C}-E_{1}-2 E_{2}, \\
\Lambda_{3}=-\bar{C}-E_{1}-E_{2}, \\
\Lambda_{1} \cdot \bar{C} \sim \mathcal{L}-2 \bar{p}_{1}-\bar{p}_{2} \sim-\bar{p}_{1}, \\
\Lambda_{2} \cdot \bar{C} \sim \mathcal{L}-\bar{p}_{1}-2 \bar{p}_{2} \sim \bar{p}_{1}, \\
h^{1}\left(\bar{C}, \mathcal{O}_{\bar{C}}\left(a \Lambda_{1}+b \Lambda_{2}\right)\right)=h^{1}\left(\bar{C}, \mathcal{O}_{\bar{C}}\left((b-a) \bar{p}_{1}\right)= \begin{cases}1, & a=b, \\
0, & \text { otherwise. }\end{cases} \right.
\end{gathered}
$$

Suppose that $\mathcal{N}$ is a numerically effective line bundle on $Y$. We have exact sequences

$0 \rightarrow \mathcal{O}_{\bar{C}}\left(-n\left(\bar{C}+F_{1}+F_{2}\right)\right) \otimes \mathcal{N} \rightarrow \mathcal{O}_{n\left(\bar{C}+F_{1}+F_{2}\right)+\bar{C}} \otimes \mathcal{N} \rightarrow \mathcal{O}_{n\left(\bar{C}+F_{1}+F_{2}\right)} \otimes \mathcal{N} \rightarrow 0$

for $n \geq 1$ and

$$
\begin{aligned}
0 \rightarrow \mathcal{O}_{F_{1}+F_{2}}\left(-n\left(\bar{C}+F_{1}+F_{2}\right)-\bar{C}\right) \otimes \mathcal{N} \rightarrow \mathcal{O}_{(n+1)\left(\bar{C}+F_{1}+F_{2}\right)} \otimes \mathcal{N} & \\
& \rightarrow \mathcal{O}_{n\left(\bar{C}+F_{1}+F_{2}\right)+\bar{C}} \otimes \mathcal{N} \rightarrow 0
\end{aligned}
$$

for $n \geq 0$. Since

$$
H^{1}(Y, \mathcal{N}) \cong \lim _{\leftarrow} H^{1}\left(Y, \mathcal{O}_{n\left(\bar{C}+F_{1}+F_{2}\right)} \otimes \mathcal{N}\right),
$$


we have

$$
H^{1}(Y, \mathcal{N}) \cong H^{1}\left(\bar{C}, \mathcal{O}_{\bar{C}} \otimes \mathcal{N}\right)
$$

from the above exact sequences. Thus

$$
h^{1}\left(Y, \mathcal{O}_{Y}\left(a \Lambda_{1}+b \Lambda_{2}\right)\right)= \begin{cases}1, & a=b \\ 0, & \text { otherwise. }\end{cases}
$$

Thus if $i, j, k>0$ and $i+j=3 k$, we have

$h^{1}\left(Y, \mathcal{O}_{Y}\left(-i E_{1}-j E_{2}-k \bar{C}\right)=h^{1}\left(Y, \mathcal{O}_{Y}\left((i-1) \Lambda_{1}+(2 k-i) \Lambda_{2}\right)= \begin{cases}1 & \text { if } i=j, \\ 0 & \text { otherwise. }\end{cases}\right.\right.$

\section{REFERENCES}

1. S. Abhyankar, Local uniformization on algebraic surfaces over ground fields of characteristic $p \neq 0$, Annals of Math. (2) 63 (1956), 491-526. MR 17:1134d

2. M. Artin, Some numerical criteria for contractability of curves on algebraic surfaces, Amer. J. Math. 84 (1962), 485-496. MR 26:3704

3. A. Borel, Linear Algebraic Groups, second ed., Springer-Verlag, New York, 1991. MR 92d:20001

4. C. Bănică and O. Stănăçilă, Algebraic methods in the global theory of complex spaces, John Wiley and Sons, New York (1976). MR 57:3420

5. A. Campillo, F. Delgado, and S. M. Gusein-Zade, The Alexander polynomial of a plane curve singularity, and the ring of functions on the curve (Russian), Uspekhi Mat. Nauk 54 (1999), no. 3, (327), 157-158; transl. in Russian Math. Surveys 54 (1999), 634-635. MR 2000h:32043

6. A. Campillo and C. Galindo, The Poincaré series associated with finitely many monomial valuations, preprint.

7. V. Cossart, O. Piltant, and A. Reguera, Divisorial valuations on rational surface singularities, Fields Inst. Comm. Vol. 32: "Valuation theory and its applications", Amer. Math. Soc., Providence, RI, 2002, 89-101.

8. S. D. Cutkosky, On unique and almost unique factorization of complete ideals II, Inventiones Math. 98 (1989), 59-74. MR 90j:14016a

9. S. D. Cutkosky and V. Srinivas, On a problem of Zariski on dimensions of linear systems, Annals of Math. (2) 137 (1993), 531-559. MR 94g:14001

10. H. Grauert, Über Modifikationen und exzeptionelle analytische Mengen, Math. Ann. 146 (1962), 331-368. MR 25:583

11. A. Grothendieck, Téchnique de descente et théorèmes d'éxistence en géométrie algébrique, VI, Séminaire Bourbaki, 1961/62, Exposé 236, Secrétariat Math., Paris, 1962. MR 26:3561

12. A. Grothendieck and J. Dieudonné, Eléments de Géometrie Algébrique, Inst. Hautes Etudes Sci. Publ. Math. 4, 8, 11, 17, 20, 24, 28, 32. MR 36:177a. MR 36:177b, MR 36:177c, MR 29:1210, MR 30:3885, MR 33:7330 MR 36:178, MR 39:220

13. A. Grothendieck and J. P. Murre, The tame fundamental group of a formal neighbourhood of a divisor with normal crossings on a scheme, Lecture Notes in Math. 208, Springer-Verlag, New York (1971). MR 47:5000

14. R. Hartshorne, Algebraic Geometry, Graduate Texts in Mathematics 52, Springer-Verlag, New York (1977). MR 57:3116

15. M. Kato, Riemann-Roch theorem for strongly pseudoconvex manifolds of dimension 2, Math. Ann. 222 (1976), 243-250. MR 54:594

16. G. Kempf, F. Knudsen, D. Mumford, and B. Saint-Donat, Toroidal embeddings I, Lecture Notes in Math. 339, Springer-Verlag, New York (1973). MR 49:299

17. H. Laufer, On rational singularities, Amer. J. Math. 94 (1972), 597-608. MR 48:8837

18. C. Lech, A note on recurring series, Arkiv Mat. 2 (1953), 417-421. MR 15:104e

19. J. Lipman, Rational singularities with applications to algebraic surfaces and unique factorization, Inst. Hautes Etudes Sci. Publ. Math. 36 (1969), 195-279. MR 43:1986

20. T. Matsusaka, The criteria for algebraic equivalence and the torsion group, Amer. J. Math. 79 (1957), 53-66. MR 18:602a

21. M. McQuillan, Division points on semi-abelian varieties, Invent. Math. 120 (1995), 143-159. MR 96b:14020 
22. M. Morales, Calcul de quelques invariants des singularité de surface normale, in Knots, braids and singularities (Plans-sur-Bex, 1982), 191-203, Monograph Enseign. Math. 31, 1983. MR 85j:14003

23. D. Mumford, The topology of normal singularities of an algebraic surface and a criterion for simplicity, Inst. Hautes Etudes Sci. Publ. Math. 9 (1961), 5-22. MR 27:3643

24. J. P. Murre, On contravariant functors from the category of preschemes over a field into the category of abelian groups, Inst. Hautes Etudes Sci. Publ. Math. 23 (1964), 5-43. MR 34:5836

25. T. Oda, Convex bodies and algebraic geometry, Springer-Verlag, Berlin (1988). MR 88m:14038

26. J. P. Serre, Algebraic Groups and Class Fields, Springer-Verlag, New York, 1988. MR 88i:14041

27. J. P. Serre, Géométrie algébrique et géométrie analytique, Ann. Inst. Fourier Grenoble 6 (1956), 1-42. MR 18:511a

28. R. Stanley, Combinatorics and commutative algebra, Birkhäuser, Boston (1983). MR 85b:05002

29. P. Vojta, Integral points on subvarieties of semi-abelian varieties I, Invent. Math. 126 (1996), 133-181. MR 98a:14034

30. O. Zariski, The theorem of Riemann-Roch for high multiples of an effective divisor on an algebraic surface, Annals of Math. 76 (1962), 560-616. MR 25:5065

Department of Mathematics, University of Missouri, Columbia, Missouri 65211

E-mail address: cutkoskys@missouri.edu

FB 6 Mathematik und Informatik, Universität-GHS-Essen, Postfach 103764, D-45117 Essen, Germany

E-mail address: mat300@uni-essen.de

Univeristy of Valladolid, Departamento de Algebra, Geometría y Topología, 005 VALLADOLID, Spain

E-mail address: areguera@agt.uva.es 